\title{
Synthesis of enantiomeric
}

\section{polyhydroxyalkylpyrrolidines from 1,3-dipolar cycloadducts. Evaluation as inhibitors of a}

\section{$\beta$-galactofuranosidase}

Guillermo A. Oliveira Udry, ${ }^{\dagger}$ Evangelina Repetto, ${ }^{\dagger}$ Daniel R. Vega $^{\ddagger}$ and Oscar Varela ${ }^{\dagger} *$

${ }^{\dagger}$ CIHIDECAR-CONICET-UBA, Departamento de Química Orgánica, Facultad de Ciencias Exactas y Naturales, Universidad de Buenos Aires, Pabellón 2, Ciudad Universitaria, 1428-Buenos Aires, Argentina

‡ Departamento Física de la Materia Condensada, GAIyANN-CAC-CNEA and ECyT-UNSAM, Av. Gral. Paz 1499, 1650- San Martín, Buenos Aires, Argentina 


\section{TABLE OF CONTENTS}

\section{General information}

Crystallographic data for compounds $2 a$ and $2 b$

S4-S23

Copies of ${ }^{1} \mathrm{H}$ - and ${ }^{13} \mathrm{C}-\mathrm{NMR}$ spectra of compounds 3a-3f, 4a, 4b, 4f, 5a, 5c, 6a, 7a, 10a, 11a, 12a, 13a, 14, 15a, 16a, 17a, 18a, 6b, 7b, 10b, 11b, 12b, 13b, 15b, 16b, 17b, and 18b and 2D COSY, 2D NOESY, 2D HMBC spectra of selected compounds. 


\section{General Methods}

Column chromatography was carried out with silica gel 60 (230-400 mesh). Analytical thin-layer chromatography (TLC) was carried out on silica gel 60 F254 aluminum-supported plates (layer thickness $0.2 \mathrm{~mm}$ ). The spots were visualized by exposure to UV light and by charring with sulfuric acid (5\% v/v in EtOH, containing 0.5\% p-anisaldehyde). Optical rotations were measured at $25^{\circ} \mathrm{C}$ in a $1 \mathrm{dm}$ cell, in the solvent indicated. Nuclear magnetic resonance (NMR) spectra were recorded at $500 \mathrm{MHz}\left({ }^{1} \mathrm{H}\right)$ or $125.7 \mathrm{MHz}\left({ }^{13} \mathrm{C}\right)$. Chemical shifts were calibrated to tetramethylsilane or to a residual solvent peak $\left(\mathrm{CHCl}_{3}:{ }^{1} \mathrm{H}: \delta=7.26\right.$ ppm, $\left.{ }^{13} \mathrm{C}: \delta=77.2 \mathrm{ppm}\right)$. Assignments of ${ }^{1} \mathrm{H}$ and ${ }^{13} \mathrm{C}$ NMR spectra were assisted by 2D ${ }^{1} \mathrm{H}-\mathrm{COSY}$ and $2 \mathrm{D}{ }^{1} \mathrm{H}-{ }^{13} \mathrm{C}$ HSQC or HMBC experiments. To facilitate the description of the NMR data the protons and carbons of the hydroxyalkyl substituents of the ring have been labeled with numbers carrying a prime symbol, as shown in the schemes. Each proton of the $\mathrm{AB}$ system of a $\mathrm{CH}_{2}$ group has been labeled as $\mathrm{x}$ and $\mathrm{y}$ (the use of $\mathrm{a}$ and $\mathrm{b}$ has been avoided as these descriptors are employed for atoms involved in the union of two rings). High-resolution mass spectra (HRMS) were obtained using the electrospray ionization (ESI) technique and Q-TOF detection. 


\section{Crystallographic data for compounds $2 \mathrm{a}$ and $2 \mathrm{~b}$}

\section{X-Ray single crystal structure for compound 2a}

Single crystal X-Ray diffraction data were collected at room temperature with graphite-monochromated MoKa $(\lambda=0.71073 \AA$ ) radiation. Data-collection strategy ( $\omega$-scan) and data reduction (integrated and scaled intensities) followed standard procedures implemented in the CrystAlisPro ${ }^{1}$ suite of programs as correspond for a 2-component twin. 6429 independent reflections were collected, 3991 with $\mathrm{I}>2 \sigma(\mathrm{I})$, Rint:0.029 (using HKLF 4 instruction). Data were corrected empirically for absorption employing the multi-scan method implemented in CrysAlisPro. The structure was solved using program SHELXS-97² and the data set obtained from one of the individual using HKLF4. The refinement was performed using the full-matrix LS procedure with SHELXL-2014/7 ${ }^{3}$ using HKLF5 instruction. Anisotropic displacement parameters were employed for non-hydrogen atoms. All $\mathrm{H}$ atoms were located at the stereo-chemically expected positions and they were refined using a riding model. In the final cycle of refinement C32 atom showed a large ADP due to librational effects, then RIGU instruction was applied on C31 - C32 to obtain better results.

LS weights of the form $w=1 /\left[\sigma^{2}\left(F_{\mathrm{o}}{ }^{2}\right)+(0.0785 P)^{2}\right]$ where $P=\left(F_{\mathrm{o}}{ }^{2}+2 F_{\mathrm{c}}{ }^{2}\right) / 3$, were employed. $R\left[F^{2}>2 \sigma\left(F^{2}\right)\right]=0.053, w R\left(F^{2}\right)=0.144$.

We provide here the following supplementary crystallographic information for the compound: crystal data and structure refinement results (Table S1), crystal structure and numbering scheme (Figure S1), tables of fractional coordinates and equivalent isotropic displacement parameters of the non-H atoms (Table S2), bond lengths and angles (Table S3), atomic anisotropic displacement parameters (Table S4), and hydrogen atoms positions (Table S5).

\section{X-Ray single crystal structure for compound $2 b$}

Single crystal X-Ray diffraction data were collected as described for 2a. Data-collection strategy and data reduction followed standard procedures implemented in the CrystAlisPro ${ }^{1}$ software. 7783 independent reflections were collected, 5217 with $I>2 \sigma(I)$, Rint:0.032. The 
structure was solved using program SHELXS- $97^{2}$ and refined using the full-matrix LS procedure with SHELXL-2014/7. ${ }^{3}$ Anisotropic displacement parameters were employed for non-hydrogen atoms. All $\mathrm{H}$ atoms were located at the expected positions and they were refined using a riding model. In the final cycle of refinement C16A and O20B atoms showed a large ADP due to librational effects, then RIGU instructions were applied on C15A - C16A and C20B - O21B to obtain better results. LS weights of the form $w=1 /\left[\sigma^{2}\left(F_{0}^{2}\right)+(0.0771 P)^{2}+\right.$ $0.8151 P]$ where $P=\left(F_{0}^{2}+2 F_{c}^{2}\right) / 3$, were employed. $R\left[F^{2}>2 \sigma\left(F^{2}\right)\right]=0.073, w R\left(F^{2}\right)=0.189$. We provide here the following supplementary crystallographic information for the compound: crystal data and structure refinement results (Table S6), crystal structure and numbering scheme (Figure S2), tables of fractional coordinates and equivalent isotropic displacement parameters of the non-H atoms (Table S7), bond lengths and angles (Table S8), atomic anisotropic displacement parameters (Table S9) and hydrogen atoms positions (Table S10).

1 CrysAlisPro, Oxford Diffraction Ltd., version 1.171.36.21 (release 30-01-2012 CrysAlis171.NET).

2 Sheldrick, G. M. SHELXS-97. Program for Crystal Structure Resolution. Univ. of Göttingen: Göttingen, Germany, 1997. See also: Sheldrick, G. M. Acta Crystallogr. A46 1990, 467.

3 Sheldrick, G. M. SHELXL-97. Program for Crystal Structures Analysis. Univ. of Göttingen: Göttingen, Germany, 1997. See also: Sheldrick, G. M. Acta Crystallogr. A 64 2008, 112. 
Table S1. Crystal data and structure refinement for $\mathbf{2 a}$.

\author{
Empirical formula \\ Formula weight \\ Temperature \\ Wavelength \\ Crystal system \\ Space group \\ Unit cell dimensions \\ Volume \\ $\mathrm{Z}$ \\ Density (calculated) \\ Absorption coefficient \\ $\mathrm{F}(000)$ \\ Crystal size \\ Theta range for data collection \\ Index ranges \\ Reflections collected \\ Independent reflections \\ Completeness to theta $=25.242^{\circ}$ \\ Refinement method \\ Data / restraints / parameters \\ Goodness-of-fit on $\mathrm{F}^{2}$ \\ Final R indices [I>2sigma(I)] \\ $\mathrm{R}$ indices (all data) \\ Absolute structure parameter \\ Extinction coefficient \\ Largest diff. peak and hole
}

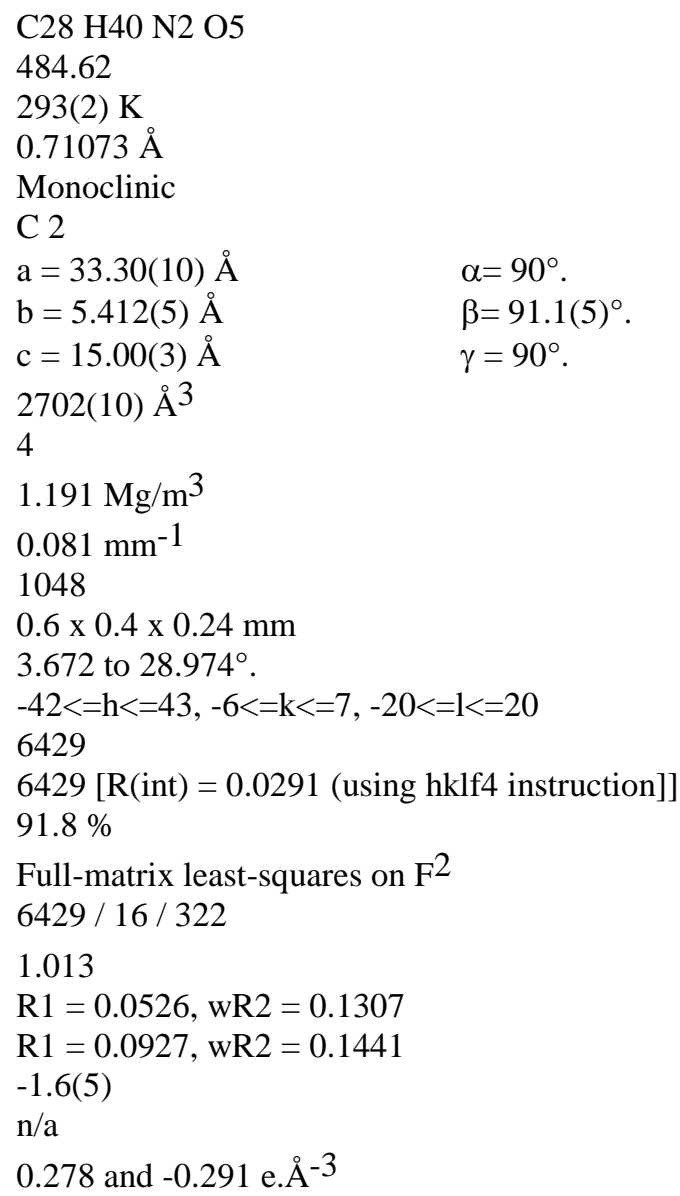


Figure S1. Crystal structure and numbering scheme for 2a. The displacement ellipsoids for the non- $\mathrm{H}$ atoms in the figure were drawn at the 50\% probability level.

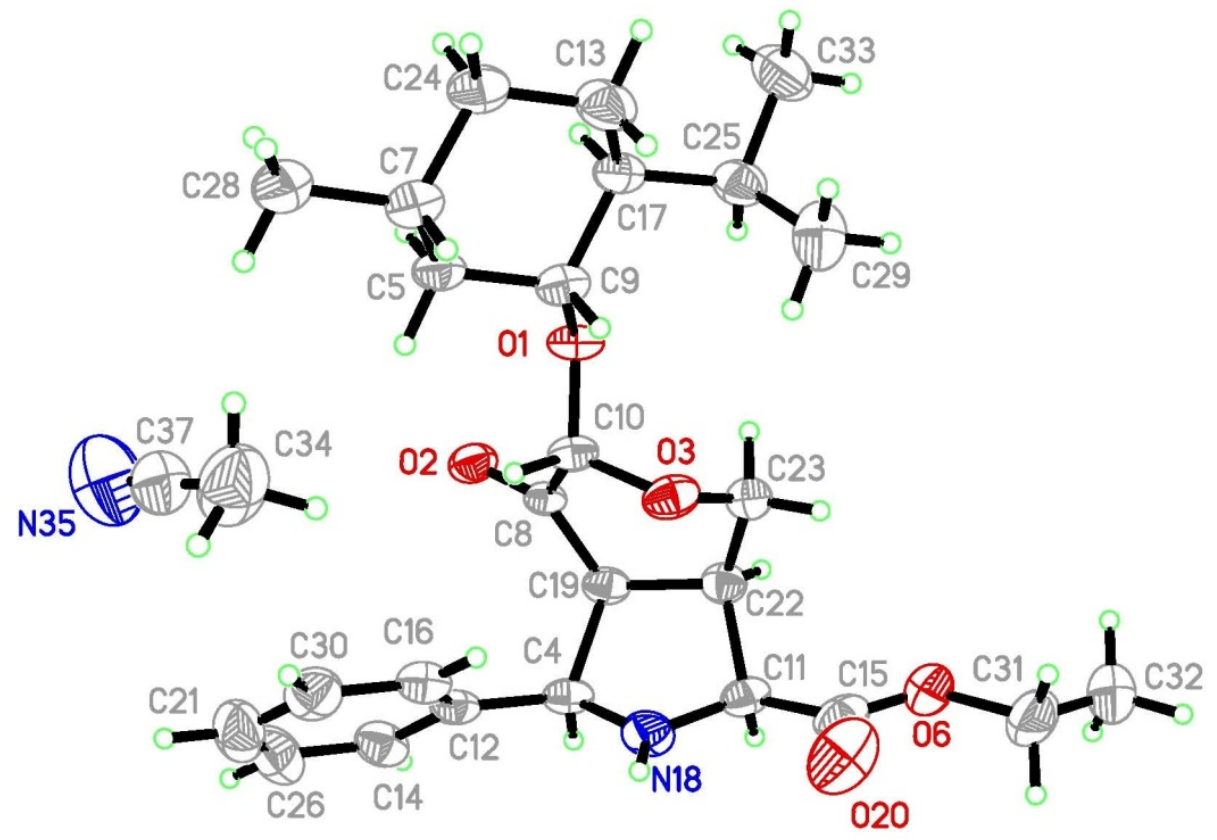


Table S2. Atomic coordinates ( $\times 10^{4}$ ) and equivalent isotropic displacement parameters $\left(\AA^{2} \times 10^{3}\right)$ for $\mathbf{2 a}$. $U(e q)$ is defined as one third of the trace of the orthogonalized $U^{i j}$ tensor.

\begin{tabular}{|c|c|c|c|c|}
\hline & $\mathrm{x}$ & $\mathrm{y}$ & $\mathrm{z}$ & $\mathrm{U}(\mathrm{eq})$ \\
\hline $\mathrm{O}(1)$ & 3290(1) & 9901(5) & $2846(2)$ & $33(1)$ \\
\hline $\mathrm{O}(2)$ & 3879(1) & $13409(5)$ & $2614(2)$ & $37(1)$ \\
\hline $\mathrm{O}(3)$ & $3677(1)$ & 8274(5) & $4013(2)$ & $38(1)$ \\
\hline C(4) & 4555(1) & 11557(7) & 4113(2) & $31(1)$ \\
\hline C(5) & $3250(1)$ & 7377(7) & $1500(2)$ & $34(1)$ \\
\hline $\mathrm{O}(6)$ & 3976(1) & $9278(6)$ & $6773(2)$ & $57(1)$ \\
\hline C(7) & 3078(1) & 5085(8) & 1058(2) & $39(1)$ \\
\hline C(8) & 3888(1) & 12021(6) & $3252(2)$ & $27(1)$ \\
\hline C(9) & 3120(1) & 7674(7) & $2460(2)$ & $32(1)$ \\
\hline$C(10)$ & $3675(1)$ & $9508(7)$ & 3186(2) & $32(1)$ \\
\hline C(11) & $4280(1)$ & 10383(7) & $5440(2)$ & $34(1)$ \\
\hline $\mathrm{C}(12)$ & 4744(1) & 10899(7) & $3229(2)$ & $33(1)$ \\
\hline $\mathrm{C}(13)$ & 2493(1) & 5458(8) & 2101(3) & $47(1)$ \\
\hline C(14) & 5015(1) & $12510(8)$ & 2854(3) & $42(1)$ \\
\hline C(15) & $4187(1)$ & 8371(8) & 6091(3) & $42(1)$ \\
\hline $\mathrm{C}(16)$ & 4661(1) & $8690(7)$ & 2797(2) & $38(1)$ \\
\hline $\mathrm{C}(17)$ & 2664(1) & 7834(7) & 2513(2) & $35(1)$ \\
\hline $\mathrm{N}(18)$ & $4538(1)$ & $9496(6)$ & $4733(2)$ & $41(1)$ \\
\hline C(19) & 4115(1) & 12643(7) & $4088(2)$ & $30(1)$ \\
\hline $\mathrm{O}(20)$ & 4292(1) & 6266(6) & 6038(2) & $67(1)$ \\
\hline C(21) & $5105(1)$ & $9770(10)$ & 1611(3) & $58(1)$ \\
\hline $\mathrm{C}(22)$ & 3921(1) & 11621(7) & 4956(2) & $32(1)$ \\
\hline C(23) & 3575(1) & 9890(8) & 4728(2) & $39(1)$ \\
\hline C(24) & 2624(1) & $5102(10)$ & 1143(3) & $49(1)$ \\
\hline C(25) & 2516(1) & 8346(8) & $3470(2)$ & $40(1)$ \\
\hline C(26) & 5192(1) & 11949(9) & 2045(3) & $56(1)$ \\
\hline C(28) & $3202(1)$ & 4849(11) & 86(3) & $54(1)$ \\
\hline C(29) & 2599(1) & $6272(10)$ & $4117(3)$ & $57(1)$ \\
\hline $\mathrm{C}(30)$ & $4840(1)$ & 8143(8) & 1991(3) & $48(1)$ \\
\hline C(33) & 2067(1) & 9029(10) & 3456(3) & $62(1)$ \\
\hline C(37) & $4281(1)$ & 3791(11) & 157(3) & $58(1)$ \\
\hline $\mathrm{N}(35)$ & $4368(2)$ & $5530(12)$ & $-210(3)$ & $91(2)$ \\
\hline C(31) & 3909(1) & 7604(13) & 7510(3) & $77(2)$ \\
\hline C(34) & $4167(2)$ & 1593(12) & $620(3)$ & $75(2)$ \\
\hline C(32) & 3719(2) & $9130(18)$ & 8238(3) & 112(3) \\
\hline
\end{tabular}


Table S3. Bond lengths $[\AA]$ and angles $\left[{ }^{\circ}\right]$ for $2 \mathbf{a}$.

\begin{tabular}{|c|c|c|c|}
\hline $\mathrm{O}(1)-\mathrm{C}(10)$ & $1.387(7)$ & $\mathrm{O}(1)-\mathrm{C}(9)$ & $1.448(5)$ \\
\hline $\mathrm{O}(2)-\mathrm{C}(8)$ & $1.216(4)$ & $\mathrm{O}(3)-\mathrm{C}(10)$ & $1.408(5)$ \\
\hline $\mathrm{O}(3)-\mathrm{C}(23)$ & $1.430(5)$ & C(4)-N(18) & $1.454(5)$ \\
\hline C(4)-C(12) & $1.521(7)$ & C(4)-C(19) & $1.579(6)$ \\
\hline $\mathrm{C}(4)-\mathrm{H}(4)$ & 0.9800 & C(5)-C(7) & $1.514(6)$ \\
\hline C(5)-C(9) & $1.520(6)$ & C(5)-H(5A) & 0.9700 \\
\hline C(5)-H(5B) & 0.9700 & $\mathrm{O}(6)-\mathrm{C}(15)$ & $1.345(7)$ \\
\hline $\mathrm{O}(6)-\mathrm{C}(31)$ & $1.450(6)$ & C(7)-C(24) & $1.517(7)$ \\
\hline C(7)-C(28) & $1.529(6)$ & $\mathrm{C}(7)-\mathrm{H}(7)$ & 0.9800 \\
\hline C(8)-C(19) & $1.489(8)$ & C(8)-C(10) & $1.536(5)$ \\
\hline C(9)-C(17) & $1.521(6)$ & $\mathrm{C}(9)-\mathrm{H}(9)$ & 0.9800 \\
\hline $\mathrm{C}(10)-\mathrm{H}(10)$ & 0.9800 & C(11)-N(18) & $1.458(7)$ \\
\hline C(11)-C(15) & $1.498(6)$ & C(11)-C(22) & $1.542(7)$ \\
\hline C(11)-H(11) & 0.9800 & C(12)-C(14) & $1.383(6)$ \\
\hline C(12)-C(16) & $1.385(5)$ & C(13)-C(24) & $1.523(7)$ \\
\hline C(13)-C(17) & $1.532(6)$ & C(13)-H(13A) & 0.9700 \\
\hline C(13)-H(13B) & 0.9700 & C(14)-C(26) & $1.393(7)$ \\
\hline $\mathrm{C}(14)-\mathrm{H}(14)$ & 0.9300 & C(15)-O(20) & $1.195(6)$ \\
\hline C(16)-C(30) & $1.390(7)$ & $\mathrm{C}(16)-\mathrm{H}(16)$ & 0.9300 \\
\hline C(17)-C(25) & 1.551(7) & $\mathrm{C}(17)-\mathrm{H}(17)$ & 0.9800 \\
\hline N(18)-H(18) & 0.8600 & C(19)-C(22) & $1.565(7)$ \\
\hline C(19)-H(19) & 0.9800 & C(21)-C(26) & $1.375(7)$ \\
\hline C(21)-C(30) & $1.378(7)$ & C(21)-H(21) & 0.9300 \\
\hline C(22)-C(23) & $1.517(6)$ & C(22)-H(22) & 0.9800 \\
\hline C(23)-H(23A) & 0.9700 & C(23)-H(23B) & 0.9700 \\
\hline C(24)-H(24A) & 0.9700 & C(24)-H(24B) & 0.9700 \\
\hline C(25)-C(29) & $1.507(7)$ & C(25)-C(33) & $1.538(7)$ \\
\hline C(25)-H(25) & 0.9800 & C(26)-H(26) & 0.9300 \\
\hline $\mathrm{C}(28)-\mathrm{H}(28 \mathrm{~A})$ & 0.9600 & $\mathrm{C}(28)-\mathrm{H}(28 \mathrm{~B})$ & 0.9600 \\
\hline C(28)-H(28C) & 0.9600 & C(29)-H(29A) & 0.9600 \\
\hline C(29)-H(29B) & 0.9600 & C(29)-H(29C) & 0.9600 \\
\hline $\mathrm{C}(30)-\mathrm{H}(30)$ & 0.9300 & C(33)-H(33A) & 0.9600 \\
\hline C(33)-H(33B) & 0.9600 & C(33)-H(33C) & 0.9600 \\
\hline $\mathrm{C}(37)-\mathrm{N}(35)$ & $1.130(7)$ & C(37)-C(34) & $1.433(9)$ \\
\hline C(31)-C(32) & $1.517(10)$ & C(31)-H(31A) & 0.9700 \\
\hline C(31)-H(31B) & 0.9700 & C(34)-H(34A) & 0.9600 \\
\hline C(34)-H(34B) & 0.9600 & C(34)-H(34C) & 0.9600 \\
\hline C(32)-H(32A) & 0.9600 & C(32)-H(32B) & 0.9600 \\
\hline C(32)-H(32C) & 0.9600 & & \\
\hline C(10)-O(1)-C(9) & 111.8(3) & C(10)-O(3)-C(23) & 111.9(3) \\
\hline N(18)-C(4)-C(12) & 113.6(3) & N(18)-C(4)-C(19) & 104.6(4) \\
\hline C(12)-C(4)-C(19) & 117.8(5) & N(18)-C(4)-H(4) & 106.7 \\
\hline C(12)-C(4)-H(4) & 106.7 & C(19)-C(4)-H(4) & 106.7 \\
\hline $\mathrm{C}(7)-\mathrm{C}(5)-\mathrm{C}(9)$ & $112.8(3)$ & $\mathrm{C}(7)-\mathrm{C}(5)-\mathrm{H}(5 \mathrm{~A})$ & 109.0 \\
\hline C(9)-C(5)-H(5A) & 109.0 & C(7)-C(5)-H(5B) & 109.0 \\
\hline C(9)-C(5)-H(5B) & 109.0 & H(5A)-C(5)-H(5B) & 107.8 \\
\hline $\mathrm{C}(15)-\mathrm{O}(6)-\mathrm{C}(31)$ & $116.3(4)$ & $\mathrm{C}(5)-\mathrm{C}(7)-\mathrm{C}(24)$ & 109.1(4) \\
\hline C(5)-C(7)-C(28) & $112.2(4)$ & C(24)-C(7)-C(28) & 111.6(5) \\
\hline C(5)-C(7)-H(7) & 107.9 & C(24)-C(7)-H(7) & 107.9 \\
\hline $\mathrm{C}(28)-\mathrm{C}(7)-\mathrm{H}(7)$ & 107.9 & $\mathrm{O}(2)-\mathrm{C}(8)-\mathrm{C}(19)$ & 121.8(3) \\
\hline $\mathrm{O}(2)-\mathrm{C}(8)-\mathrm{C}(10)$ & $119.5(4)$ & C(19)-C(8)-C(10) & 118.7(3) \\
\hline O(1)-C(9)-C(5) & 110.5(3) & O(1)-C(9)-C(17) & 108.3(3) \\
\hline C(5)-C(9)-C(17) & 111.1(5) & $\mathrm{O}(1)-\mathrm{C}(9)-\mathrm{H}(9)$ & 109.0 \\
\hline $\mathrm{C}(5)-\mathrm{C}(9)-\mathrm{H}(9)$ & 109.0 & $\mathrm{C}(17)-\mathrm{C}(9)-\mathrm{H}(9)$ & 109.0 \\
\hline $\mathrm{O}(1)-\mathrm{C}(10)-\mathrm{O}(3)$ & $112.7(4)$ & $\mathrm{O}(1)-\mathrm{C}(10)-\mathrm{C}(8)$ & 108.1(3) \\
\hline O(3)-C(10)-C(8) & 111.7(4) & $\mathrm{O}(1)-\mathrm{C}(10)-\mathrm{H}(10)$ & 108.1 \\
\hline $\mathrm{O}(3)-\mathrm{C}(10)-\mathrm{H}(10)$ & 108.1 & $\mathrm{C}(8)-\mathrm{C}(10)-\mathrm{H}(10)$ & 108.1 \\
\hline N(18)-C(11)-C(15) & 111.5(4) & N(18)-C(11)-C(22) & 105.2(3) \\
\hline C(15)-C(11)-C(22) & $117.0(4)$ & N(18)-C(11)-H(11) & 107.6 \\
\hline $\mathrm{C}(15)-\mathrm{C}(11)-\mathrm{H}(11)$ & 107.6 & $\mathrm{C}(22)-\mathrm{C}(11)-\mathrm{H}(11)$ & 107.6 \\
\hline C(14)-C(12)-C(16) & $118.7(4)$ & $\mathrm{C}(14)-\mathrm{C}(12)-\mathrm{C}(4)$ & 119.4(4) \\
\hline C(16)-C(12)-C(4) & 121.9(3) & C(24)-C(13)-C(17) & 112.1(4) \\
\hline $\mathrm{C}(24)-\mathrm{C}(13)-\mathrm{H}(13 \mathrm{~A})$ & 109.2 & $\mathrm{C}(17)-\mathrm{C}(13)-\mathrm{H}(13 \mathrm{~A})$ & 109.2 \\
\hline C(24)-C(13)-H(13B) & 109.2 & $\mathrm{C}(17)-\mathrm{C}(13)-\mathrm{H}(13 \mathrm{~B})$ & 109.2 \\
\hline $\mathrm{H}(13 \mathrm{~A})-\mathrm{C}(13)-\mathrm{H}(13 \mathrm{~B})$ & 107.9 & C(12)-C(14)-C(26) & 120.5(5) \\
\hline $\mathrm{C}(12)-\mathrm{C}(14)-\mathrm{H}(14)$ & 119.7 & C(26)-C(14)-H(14) & 119.7 \\
\hline $\mathrm{O}(20)-\mathrm{C}(15)-\mathrm{O}(6)$ & $123.8(4)$ & $\mathrm{O}(20)-\mathrm{C}(15)-\mathrm{C}(11)$ & 125.8(4) \\
\hline
\end{tabular}




\begin{tabular}{|c|c|c|c|}
\hline $\mathrm{O}(6)-\mathrm{C}(15)-\mathrm{C}(11)$ & $110.4(4)$ & $\mathrm{C}(12)-\mathrm{C}(16)-\mathrm{C}(30)$ & $120.4(4)$ \\
\hline $\mathrm{C}(12)-\mathrm{C}(16)-\mathrm{H}(16)$ & 119.8 & $\mathrm{C}(30)-\mathrm{C}(16)-\mathrm{H}(16)$ & 119.8 \\
\hline C(9)-C(17)-C(13) & 107.1(4) & C(9)-C(17)-C(25) & $113.3(5)$ \\
\hline $\mathrm{C}(13)-\mathrm{C}(17)-\mathrm{C}(25)$ & 113.6(3) & $\mathrm{C}(9)-\mathrm{C}(17)-\mathrm{H}(17)$ & 107.5 \\
\hline $\mathrm{C}(13)-\mathrm{C}(17)-\mathrm{H}(17)$ & 107.5 & $\mathrm{C}(25)-\mathrm{C}(17)-\mathrm{H}(17)$ & 107.5 \\
\hline $\mathrm{C}(4)-\mathrm{N}(18)-\mathrm{C}(11)$ & 104.1(3) & C(4)-N(18)-H(18) & 128.0 \\
\hline $\mathrm{C}(11)-\mathrm{N}(18)-\mathrm{H}(18)$ & 128.0 & $\mathrm{C}(8)-\mathrm{C}(19)-\mathrm{C}(22)$ & $114.2(3)$ \\
\hline C(8)-C(19)-C(4) & $113.0(4)$ & $\mathrm{C}(22)-\mathrm{C}(19)-\mathrm{C}(4)$ & $104.3(4)$ \\
\hline $\mathrm{C}(8)-\mathrm{C}(19)-\mathrm{H}(19)$ & 108.4 & $\mathrm{C}(22)-\mathrm{C}(19)-\mathrm{H}(19)$ & 108.4 \\
\hline $\mathrm{C}(4)-\mathrm{C}(19)-\mathrm{H}(19)$ & 108.4 & $\mathrm{C}(26)-\mathrm{C}(21)-\mathrm{C}(30)$ & $118.9(4)$ \\
\hline $\mathrm{C}(26)-\mathrm{C}(21)-\mathrm{H}(21)$ & 120.6 & $\mathrm{C}(30)-\mathrm{C}(21)-\mathrm{H}(21)$ & 120.6 \\
\hline$C(23)-C(22)-C(11)$ & $114.6(4)$ & C(23)-C(22)-C(19) & $110.7(4)$ \\
\hline $\mathrm{C}(11)-\mathrm{C}(22)-\mathrm{C}(19)$ & $102.4(4)$ & $\mathrm{C}(23)-\mathrm{C}(22)-\mathrm{H}(22)$ & 109.6 \\
\hline $\mathrm{C}(11)-\mathrm{C}(22)-\mathrm{H}(22)$ & 109.6 & $\mathrm{C}(19)-\mathrm{C}(22)-\mathrm{H}(22)$ & 109.6 \\
\hline $\mathrm{O}(3)-\mathrm{C}(23)-\mathrm{C}(22)$ & $110.9(4)$ & $\mathrm{O}(3)-\mathrm{C}(23)-\mathrm{H}(23 \mathrm{~A})$ & 109.5 \\
\hline $\mathrm{C}(22)-\mathrm{C}(23)-\mathrm{H}(23 \mathrm{~A})$ & 109.5 & $\mathrm{O}(3)-\mathrm{C}(23)-\mathrm{H}(23 \mathrm{~B})$ & 109.5 \\
\hline $\mathrm{C}(22)-\mathrm{C}(23)-\mathrm{H}(23 \mathrm{~B})$ & 109.5 & $\mathrm{H}(23 \mathrm{~A})-\mathrm{C}(23)-\mathrm{H}(23 \mathrm{~B})$ & 108.0 \\
\hline $\mathrm{C}(7)-\mathrm{C}(24)-\mathrm{C}(13)$ & $112.6(5)$ & $\mathrm{C}(7)-\mathrm{C}(24)-\mathrm{H}(24 \mathrm{~A})$ & 109.1 \\
\hline $\mathrm{C}(13)-\mathrm{C}(24)-\mathrm{H}(24 \mathrm{~A})$ & 109.1 & C(7)-C(24)-H(24B) & 109.1 \\
\hline $\mathrm{C}(13)-\mathrm{C}(24)-\mathrm{H}(24 \mathrm{~B})$ & 109.1 & $\mathrm{H}(24 \mathrm{~A})-\mathrm{C}(24)-\mathrm{H}(24 \mathrm{~B})$ & 107.8 \\
\hline C(29)-C(25)-C(33) & $110.8(4)$ & $\mathrm{C}(29)-\mathrm{C}(25)-\mathrm{C}(17)$ & $113.8(3)$ \\
\hline C(33)-C(25)-C(17) & $111.0(5)$ & $\mathrm{C}(29)-\mathrm{C}(25)-\mathrm{H}(25)$ & 106.9 \\
\hline $\mathrm{C}(33)-\mathrm{C}(25)-\mathrm{H}(25)$ & 106.9 & $\mathrm{C}(17)-\mathrm{C}(25)-\mathrm{H}(25)$ & 106.9 \\
\hline $\mathrm{C}(21)-\mathrm{C}(26)-\mathrm{C}(14)$ & $120.7(4)$ & $\mathrm{C}(21)-\mathrm{C}(26)-\mathrm{H}(26)$ & 119.7 \\
\hline C(14)-C(26)-H(26) & 119.7 & $\mathrm{C}(7)-\mathrm{C}(28)-\mathrm{H}(28 \mathrm{~A})$ & 109.5 \\
\hline $\mathrm{C}(7)-\mathrm{C}(28)-\mathrm{H}(28 \mathrm{~B})$ & 109.5 & $\mathrm{H}(28 \mathrm{~A})-\mathrm{C}(28)-\mathrm{H}(28 \mathrm{~B})$ & 109.5 \\
\hline $\mathrm{C}(7)-\mathrm{C}(28)-\mathrm{H}(28 \mathrm{C})$ & 109.5 & $\mathrm{H}(28 \mathrm{~A})-\mathrm{C}(28)-\mathrm{H}(28 \mathrm{C})$ & 109.5 \\
\hline $\mathrm{H}(28 \mathrm{~B})-\mathrm{C}(28)-\mathrm{H}(28 \mathrm{C})$ & 109.5 & $\mathrm{C}(25)-\mathrm{C}(29)-\mathrm{H}(29 \mathrm{~A})$ & 109.5 \\
\hline $\mathrm{C}(25)-\mathrm{C}(29)-\mathrm{H}(29 \mathrm{~B})$ & 109.5 & $\mathrm{H}(29 \mathrm{~A})-\mathrm{C}(29)-\mathrm{H}(29 \mathrm{~B})$ & 109.5 \\
\hline $\mathrm{C}(25)-\mathrm{C}(29)-\mathrm{H}(29 \mathrm{C})$ & 109.5 & $\mathrm{H}(29 \mathrm{~A})-\mathrm{C}(29)-\mathrm{H}(29 \mathrm{C})$ & 109.5 \\
\hline $\mathrm{H}(29 \mathrm{~B})-\mathrm{C}(29)-\mathrm{H}(29 \mathrm{C})$ & 109.5 & $\mathrm{C}(21)-\mathrm{C}(30)-\mathrm{C}(16)$ & $120.8(5)$ \\
\hline $\mathrm{C}(21)-\mathrm{C}(30)-\mathrm{H}(30)$ & 119.6 & $\mathrm{C}(16)-\mathrm{C}(30)-\mathrm{H}(30)$ & 119.6 \\
\hline C(25)-C(33)-H(33A) & 109.5 & $\mathrm{C}(25)-\mathrm{C}(33)-\mathrm{H}(33 \mathrm{~B})$ & 109.5 \\
\hline $\mathrm{H}(33 \mathrm{~A})-\mathrm{C}(33)-\mathrm{H}(33 \mathrm{~B})$ & 109.5 & $\mathrm{C}(25)-\mathrm{C}(33)-\mathrm{H}(33 \mathrm{C})$ & 109.5 \\
\hline $\mathrm{H}(33 \mathrm{~A})-\mathrm{C}(33)-\mathrm{H}(33 \mathrm{C})$ & 109.5 & $\mathrm{H}(33 \mathrm{~B})-\mathrm{C}(33)-\mathrm{H}(33 \mathrm{C})$ & 109.5 \\
\hline N(35)-C(37)-C(34) & 179.4(6) & $\mathrm{O}(6)-\mathrm{C}(31)-\mathrm{C}(32)$ & $106.3(6)$ \\
\hline $\mathrm{O}(6)-\mathrm{C}(31)-\mathrm{H}(31 \mathrm{~A})$ & 110.5 & $\mathrm{C}(32)-\mathrm{C}(31)-\mathrm{H}(31 \mathrm{~A})$ & 110.5 \\
\hline $\mathrm{O}(6)-\mathrm{C}(31)-\mathrm{H}(31 \mathrm{~B})$ & 110.5 & $\mathrm{C}(32)-\mathrm{C}(31)-\mathrm{H}(31 \mathrm{~B})$ & 110.5 \\
\hline $\mathrm{H}(31 \mathrm{~A})-\mathrm{C}(31)-\mathrm{H}(31 \mathrm{~B})$ & 108.7 & $\mathrm{C}(37)-\mathrm{C}(34)-\mathrm{H}(34 \mathrm{~A})$ & 109.5 \\
\hline $\mathrm{C}(37)-\mathrm{C}(34)-\mathrm{H}(34 \mathrm{~B})$ & 109.5 & $\mathrm{H}(34 \mathrm{~A})-\mathrm{C}(34)-\mathrm{H}(34 \mathrm{~B})$ & 109.5 \\
\hline C(37)-C(34)-H(34C) & 109.5 & $\mathrm{H}(34 \mathrm{~A})-\mathrm{C}(34)-\mathrm{H}(34 \mathrm{C})$ & 109.5 \\
\hline $\mathrm{H}(34 \mathrm{~B})-\mathrm{C}(34)-\mathrm{H}(34 \mathrm{C})$ & 109.5 & $\mathrm{C}(31)-\mathrm{C}(32)-\mathrm{H}(32 \mathrm{~A})$ & 109.5 \\
\hline $\mathrm{C}(31)-\mathrm{C}(32)-\mathrm{H}(32 \mathrm{~B})$ & 109.5 & $\mathrm{H}(32 \mathrm{~A})-\mathrm{C}(32)-\mathrm{H}(32 \mathrm{~B})$ & 109.5 \\
\hline $\mathrm{C}(31)-\mathrm{C}(32)-\mathrm{H}(32 \mathrm{C})$ & 109.5 & $\mathrm{H}(32 \mathrm{~A})-\mathrm{C}(32)-\mathrm{H}(32 \mathrm{C})$ & 109.5 \\
\hline $\mathrm{H}(32 \mathrm{~B})-\mathrm{C}(32)-\mathrm{H}(32 \mathrm{C})$ & 109.5 & & \\
\hline
\end{tabular}


Table S4. Anisotropic displacement parameters $\left(\AA^{2} \times 10^{3}\right)$ for 2a. The anisotropic displacement factor exponent takes the form: $-2 \pi^{2}\left[h^{2} a^{* 2} U^{11}+\ldots+2 h k^{*} b^{*} U^{12}\right]$

\begin{tabular}{|c|c|c|c|c|c|c|}
\hline & $\mathrm{U}^{11}$ & $\mathrm{U}^{22}$ & $\mathrm{U}^{33}$ & $\mathrm{U}^{23}$ & $\mathrm{U}^{13}$ & $\mathrm{U}^{12}$ \\
\hline $\mathrm{O}(1)$ & $24(1)$ & $28(1)$ & $46(1)$ & $-2(1)$ & $-12(1)$ & 1(1) \\
\hline $\mathrm{O}(2)$ & $33(1)$ & 41(2) & $38(1)$ & $8(1)$ & $-7(1)$ & $2(1)$ \\
\hline $\mathrm{O}(3)$ & 39(1) & 29(1) & $45(2)$ & $4(1)$ & $-12(1)$ & $-1(1)$ \\
\hline C(4) & $25(2)$ & $28(2)$ & $41(2)$ & $-1(2)$ & $-9(2)$ & $-1(1)$ \\
\hline$C(5)$ & $27(2)$ & $35(2)$ & $41(2)$ & $7(2)$ & $-8(2)$ & $0(2)$ \\
\hline$O(6)$ & $49(2)$ & 75(2) & $46(2)$ & $24(2)$ & $-2(1)$ & $1(2)$ \\
\hline C(7) & $37(2)$ & $39(2)$ & $40(2)$ & $0(2)$ & $-9(2)$ & $-1(2)$ \\
\hline C(8) & 20(1) & $29(2)$ & $31(2)$ & 2(2) & $-2(1)$ & $6(1)$ \\
\hline C(9) & $32(2)$ & 22(2) & $40(2)$ & 3(2) & $-9(2)$ & $-3(1)$ \\
\hline$C(10)$ & $26(2)$ & $31(2)$ & $37(2)$ & 2(2) & $-8(1)$ & $6(2)$ \\
\hline$C(11)$ & $33(2)$ & $32(2)$ & $36(2)$ & 2(2) & $-10(2)$ & $-3(2)$ \\
\hline$C(12)$ & $24(2)$ & $34(2)$ & $41(2)$ & $7(2)$ & $-7(2)$ & $5(2)$ \\
\hline C(13) & $36(2)$ & 50(3) & $55(3)$ & $-8(2)$ & $0(2)$ & $-13(2)$ \\
\hline$C(14)$ & $34(2)$ & $37(2)$ & $56(3)$ & $6(2)$ & $1(2)$ & $2(2)$ \\
\hline C(15) & $41(2)$ & $42(2)$ & $42(2)$ & $6(2)$ & $-18(2)$ & $-13(2)$ \\
\hline$C(16)$ & $30(2)$ & $37(2)$ & $46(2)$ & 1(2) & $-5(2)$ & $3(2)$ \\
\hline$C(17)$ & $27(2)$ & $35(2)$ & $42(2)$ & $4(2)$ & $-6(2)$ & $-2(2)$ \\
\hline $\mathrm{N}(18)$ & $36(2)$ & $35(2)$ & $51(2)$ & 12(2) & $3(1)$ & $14(2)$ \\
\hline C(19) & $26(2)$ & 23(2) & $40(2)$ & $3(2)$ & $-4(2)$ & 1(1) \\
\hline $\mathrm{O}(20)$ & 101(3) & $31(2)$ & $67(2)$ & $9(2)$ & $-11(2)$ & $-8(2)$ \\
\hline$C(21)$ & 61(3) & 60(3) & $52(3)$ & $5(3)$ & $15(2)$ & $22(3)$ \\
\hline$C(22)$ & $30(2)$ & $32(2)$ & $35(2)$ & $-2(2)$ & $-1(1)$ & $2(2)$ \\
\hline C(23) & 31(2) & 50(2) & $36(2)$ & $7(2)$ & $-2(2)$ & $-1(2)$ \\
\hline$C(24)$ & $39(2)$ & 57(3) & $49(2)$ & $-9(2)$ & $-9(2)$ & $-14(2)$ \\
\hline C(25) & $33(2)$ & 40(2) & $46(2)$ & $-6(2)$ & $-1(2)$ & $-7(2)$ \\
\hline C(26) & $47(2)$ & 54(3) & $69(3)$ & $13(3)$ & $18(2)$ & $4(2)$ \\
\hline C(28) & $43(2)$ & 72(3) & $46(2)$ & $-10(3)$ & $-6(2)$ & $-1(2)$ \\
\hline C(29) & 69(3) & 55(3) & $47(2)$ & $0(3)$ & $10(2)$ & $-4(2)$ \\
\hline C(30) & $50(2)$ & $43(2)$ & $49(2)$ & $-1(2)$ & $-4(2)$ & $14(2)$ \\
\hline C(33) & $40(2)$ & $77(4)$ & $69(3)$ & $-11(3)$ & $9(2)$ & $-6(2)$ \\
\hline C(37) & $53(3)$ & 71(4) & $48(3)$ & $-3(3)$ & $3(2)$ & $8(3)$ \\
\hline N(35) & 93(3) & $87(4)$ & 94(4) & $3(3)$ & 27(3) & $-9(3)$ \\
\hline C(31) & $58(3)$ & $115(5)$ & $58(3)$ & $47(3)$ & $-12(2)$ & $-23(3)$ \\
\hline$C(34)$ & $92(4)$ & $75(4)$ & 59(3) & 12(3) & $3(3)$ & $25(3)$ \\
\hline C(32) & $60(3)$ & $215(9)$ & 63(3) & $58(5)$ & $18(2)$ & $24(4)$ \\
\hline
\end{tabular}


Table S5. Hydrogen coordinates $\left(\times 10^{4}\right)$ and isotropic displacement parameters $\left(\AA^{2} \times 10^{3}\right)$ for $\mathbf{2 a}$.

\begin{tabular}{|c|c|c|c|c|}
\hline & $\mathrm{x}$ & $\mathrm{y}$ & $\mathrm{z}$ & $\mathrm{U}(\mathrm{eq})$ \\
\hline $\mathrm{H}(4)$ & 4727 & 12814 & 4394 & 38 \\
\hline $\mathrm{H}(5 \mathrm{~A})$ & 3165 & 8818 & 1161 & 41 \\
\hline $\mathrm{H}(5 \mathrm{~B})$ & 3541 & 7298 & 1488 & 41 \\
\hline $\mathrm{H}(7)$ & 3182 & 3643 & 1383 & 47 \\
\hline $\mathrm{H}(9)$ & 3214 & 6246 & 2807 & 38 \\
\hline $\mathrm{H}(10)$ & 3820 & 8482 & 2761 & 38 \\
\hline $\mathrm{H}(11)$ & 4428 & 11676 & 5766 & 40 \\
\hline $\mathrm{H}(13 \mathrm{~A})$ & 2582 & 4055 & 2456 & 56 \\
\hline $\mathrm{H}(13 \mathrm{~B})$ & 2202 & 5516 & 2115 & 56 \\
\hline $\mathrm{H}(14)$ & 5080 & 13980 & 3145 & 50 \\
\hline $\mathrm{H}(16)$ & 4485 & 7565 & 3048 & 45 \\
\hline $\mathrm{H}(17)$ & 2576 & 9215 & 2135 & 41 \\
\hline $\mathrm{H}(18)$ & 4653 & 8079 & 4692 & 49 \\
\hline $\mathrm{H}(19)$ & 4132 & 14446 & 4136 & 36 \\
\hline $\mathrm{H}(21)$ & 5223 & 9400 & 1071 & 69 \\
\hline $\mathrm{H}(22)$ & 3823 & 13000 & 5315 & 38 \\
\hline $\mathrm{H}(23 \mathrm{~A})$ & 3510 & 8919 & 5249 & 47 \\
\hline $\mathrm{H}(23 \mathrm{~B})$ & 3340 & 10850 & 4558 & 47 \\
\hline $\mathrm{H}(24 \mathrm{~A})$ & 2517 & 3552 & 918 & 58 \\
\hline $\mathrm{H}(24 \mathrm{~B})$ & 2513 & 6423 & 777 & 58 \\
\hline $\mathrm{H}(25)$ & 2663 & 9795 & 3692 & 48 \\
\hline $\mathrm{H}(26)$ & 5372 & 13060 & 1796 & 68 \\
\hline $\mathrm{H}(28 \mathrm{~A})$ & 3080 & 6154 & -259 & 80 \\
\hline $\mathrm{H}(28 \mathrm{~B})$ & 3114 & 3282 & -146 & 80 \\
\hline $\mathrm{H}(28 \mathrm{C})$ & 3489 & 4963 & 52 & 80 \\
\hline $\mathrm{H}(29 \mathrm{~A})$ & 2514 & 6749 & 4701 & 86 \\
\hline $\mathrm{H}(29 \mathrm{~B})$ & 2882 & 5924 & 4135 & 86 \\
\hline $\mathrm{H}(29 \mathrm{C})$ & 2455 & 4822 & 3929 & 86 \\
\hline $\mathrm{H}(30)$ & 4780 & 6661 & 1705 & 57 \\
\hline $\mathrm{H}(33 \mathrm{~A})$ & 1911 & 7631 & 3260 & 92 \\
\hline $\mathrm{H}(33 \mathrm{~B})$ & 2022 & 10385 & 3054 & 92 \\
\hline $\mathrm{H}(33 \mathrm{C})$ & 1988 & 9497 & 4045 & 92 \\
\hline $\mathrm{H}(31 \mathrm{~A})$ & 4161 & 6898 & 7722 & 93 \\
\hline $\mathrm{H}(31 \mathrm{~B})$ & 3731 & 6270 & 7326 & 93 \\
\hline $\mathrm{H}(34 \mathrm{~A})$ & 3917 & 995 & 379 & 113 \\
\hline $\mathrm{H}(34 \mathrm{~B})$ & 4138 & 1958 & 1242 & 113 \\
\hline $\mathrm{H}(34 \mathrm{C})$ & 4371 & 354 & 551 & 113 \\
\hline $\mathrm{H}(32 \mathrm{~A})$ & 3892 & 10491 & 8391 & 169 \\
\hline $\mathrm{H}(32 \mathrm{~B})$ & 3681 & 8117 & 8755 & 169 \\
\hline $\mathrm{H}(32 \mathrm{C})$ & 3464 & 9748 & 8029 & 169 \\
\hline
\end{tabular}


Table S6. Crystal data and structure refinement for $\mathbf{2 b}$.

\author{
Empirical formula \\ Formula weight \\ Temperature \\ Wavelength \\ Crystal system \\ Space group \\ Unit cell dimensions \\ Volume \\ $\mathrm{Z}$ \\ Density (calculated) \\ Absorption coefficient \\ $\mathrm{F}(000)$ \\ Crystal size \\ Theta range for data collection \\ Index ranges \\ Reflections collected \\ Independent reflections \\ Completeness to theta $=25.242^{\circ}$ \\ Absorption correction \\ Max. and min. transmission \\ Refinement method \\ Data / restraints / parameters \\ Goodness-of-fit on $\mathrm{F}^{2}$ \\ Final R indices [I $>2 \operatorname{sigma}(\mathrm{I})]$ \\ $\mathrm{R}$ indices (all data) \\ Absolute structure parameter \\ Extinction coefficient \\ Largest diff. peak and hole
}

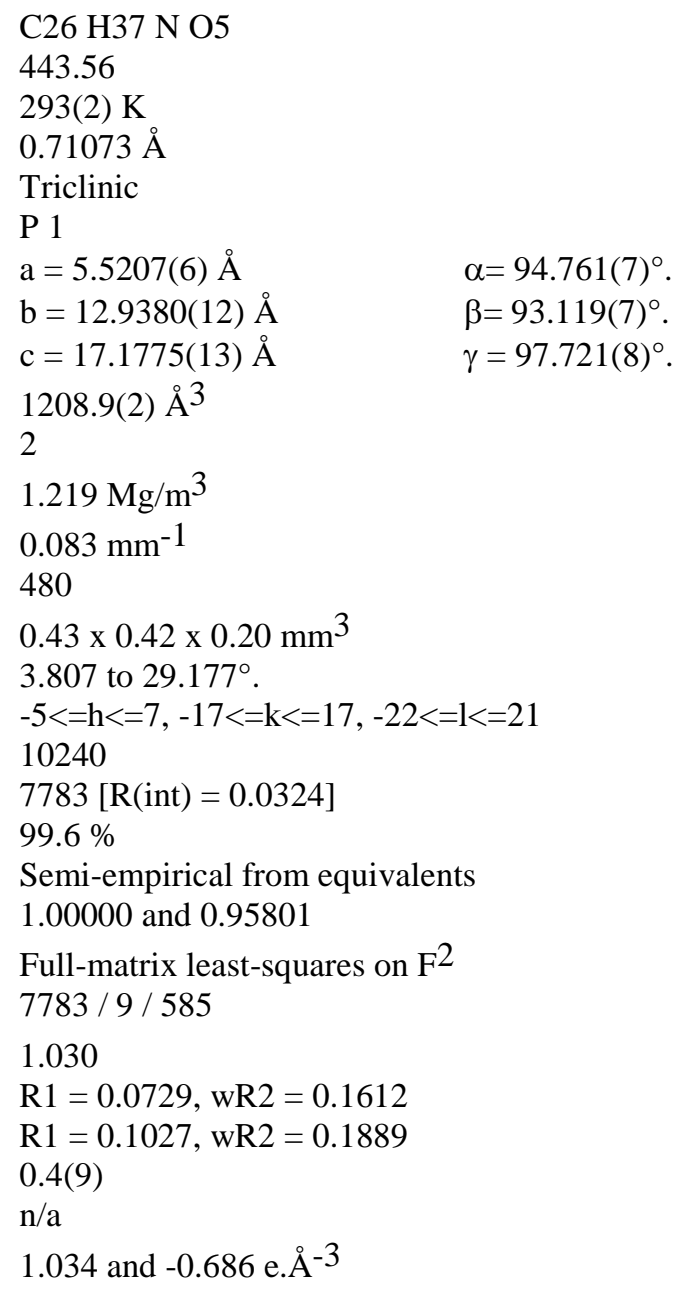


Figure S2. Crystal structure and numbering scheme for $\mathbf{2} \mathbf{b}$. The displacement ellipsoids for the non- $\mathrm{H}$ atoms in the figure were drawn at the $50 \%$ probability level.

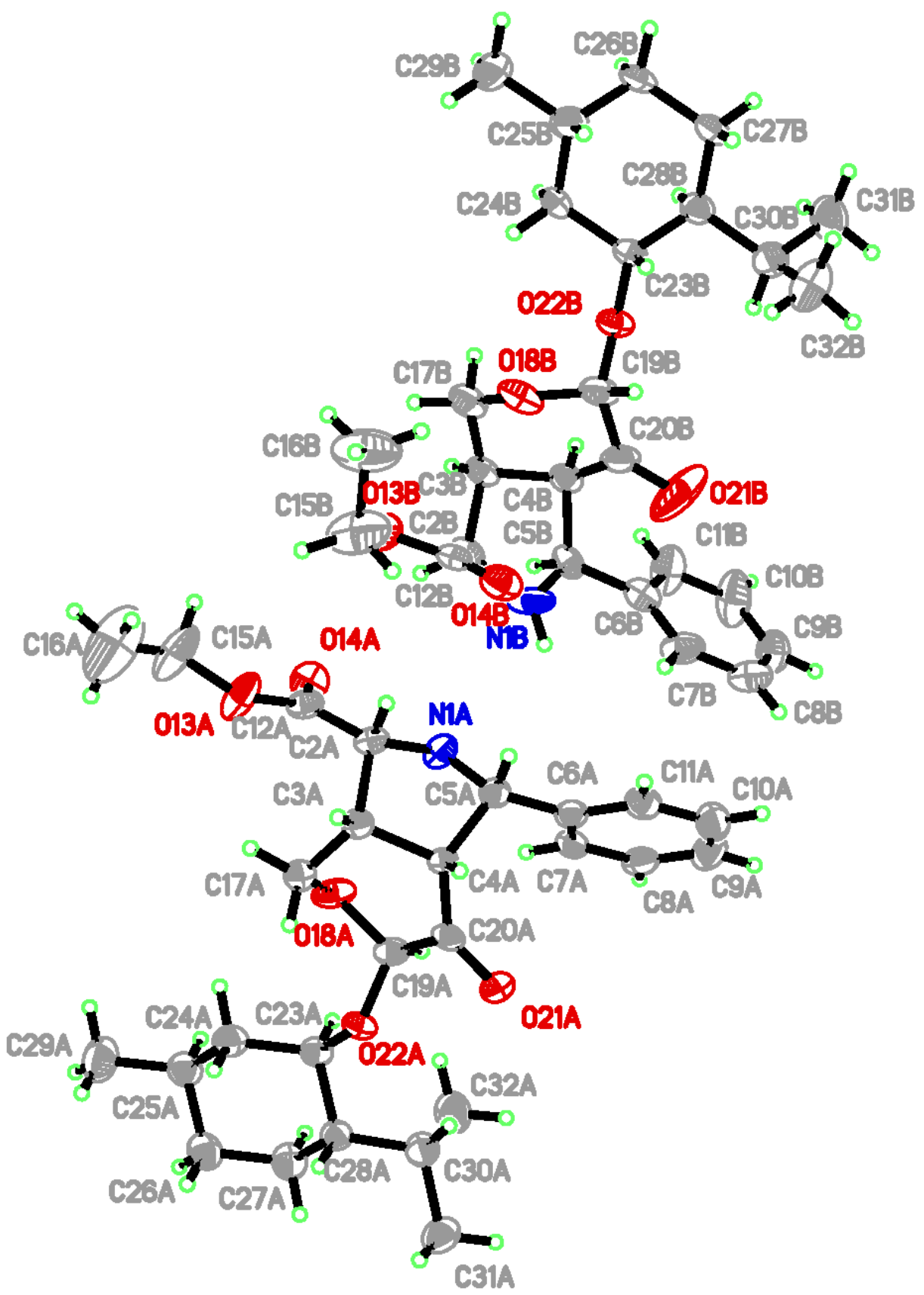


Table S7. Atomic coordinates $\left(\times 10^{4}\right)$ and equivalent isotropic displacement parameters $\left(\AA^{2} \times 10^{3}\right)$ for $\mathbf{2} \mathbf{b}$. U(eq) is defined as one third of the trace of the orthogonalized Uij tensor.

\begin{tabular}{|c|c|c|c|c|}
\hline & $\mathrm{x}$ & $\mathrm{y}$ & $\mathrm{z}$ & $\mathrm{U}(\mathrm{eq})$ \\
\hline $\mathrm{N}(1 \mathrm{~A})$ & $8540(9)$ & 4018(3) & 4188(3) & $31(1)$ \\
\hline $\mathrm{C}(2 \mathrm{~A})$ & $7620(10)$ & 2974(4) & $3825(3)$ & $26(1)$ \\
\hline$C(3 \mathrm{~A})$ & $6217(10)$ & $2417(4)$ & 4474(3) & $26(1)$ \\
\hline$C(4 A)$ & $5406(10)$ & $3345(4)$ & 4984(3) & $22(1)$ \\
\hline $\mathrm{C}(5 \mathrm{~A})$ & $6366(10)$ & 4323(4) & 4552(3) & $26(1)$ \\
\hline$C(6 A)$ & 6831(11) & 5392(4) & 5011(3) & 28(1) \\
\hline$C(7 A)$ & $8905(11)$ & 5709(4) & 5506(3) & $29(1)$ \\
\hline$C(8 A)$ & $9292(12)$ & $6700(4)$ & 5913(3) & $34(1)$ \\
\hline C(9A) & 7548(13) & 7358(5) & 5837(4) & $44(2)$ \\
\hline C(10A) & $5480(13)$ & 7066(5) & 5344(4) & $42(2)$ \\
\hline $\mathrm{C}(11 \mathrm{~A})$ & 5121(11) & 6079(4) & 4935(3) & $34(1)$ \\
\hline$C(12 A)$ & 9597(11) & $2450(4)$ & 3452(3) & $30(1)$ \\
\hline $\mathrm{O}(13 \mathrm{~A})$ & 8694(8) & 1483(3) & 3141(3) & $49(1)$ \\
\hline $\mathrm{O}(14 \mathrm{~A})$ & $11629(8)$ & 2851(3) & 3394(3) & $39(1)$ \\
\hline $\mathrm{C}(15 \mathrm{~A})$ & $10377(15)$ & $941(6)$ & $2676(5)$ & $69(3)$ \\
\hline$C(16 A)$ & $9220(20)$ & $-6(10)$ & 2361(8) & $122(5)$ \\
\hline$C(17 A)$ & 7725(11) & 1783(4) & 4961(3) & 31(1) \\
\hline$O(18 A)$ & $9681(7)$ & 2478(3) & $5410(2)$ & $36(1)$ \\
\hline C(19A) & $8739(10)$ & $3005(4)$ & 6059(3) & 28(1) \\
\hline$C(20 A)$ & 6331(11) & $3420(4)$ & 5834(3) & $26(1)$ \\
\hline $\mathrm{O}(21 \mathrm{~A})$ & 5217(8) & $3804(3)$ & $6345(2)$ & $36(1)$ \\
\hline$O(22 A)$ & 8263(7) & 2359(3) & $6660(2)$ & 28(1) \\
\hline$C(23 A)$ & 10439(10) & 2040(4) & 7031(3) & 27(1) \\
\hline$C(24 A)$ & 10699(12) & 928(5) & 6703(3) & $35(1)$ \\
\hline$C(25 A)$ & 12932(12) & $538(5)$ & 7093(4) & $37(1)$ \\
\hline$C(26 A)$ & 12729(13) & 612(5) & $7975(4)$ & $39(2)$ \\
\hline $\mathrm{C}(27 \mathrm{~A})$ & $12439(12)$ & 1717(5) & $8302(4)$ & $40(2)$ \\
\hline$C(28 A)$ & 10199(11) & $2107(4)$ & 7913(3) & $29(1)$ \\
\hline$C(29 A)$ & $13168(16)$ & $-563(5)$ & 6773(5) & $56(2)$ \\
\hline$C(30 A)$ & 9756(12) & $3187(4)$ & 8261(3) & $34(1)$ \\
\hline$C(31 \mathrm{~A})$ & 8947(13) & $3153(5)$ & $9096(4)$ & $43(2)$ \\
\hline$C(32 A)$ & $11972(14)$ & 4026(5) & $8239(4)$ & $51(2)$ \\
\hline $\mathrm{N}(1 \mathrm{~B})$ & 10187(12) & 5823(4) & 3062(3) & $49(2)$ \\
\hline$C(2 B)$ & 10203(11) & $5026(4)$ & 2419(3) & $32(1)$ \\
\hline $\mathrm{C}(3 \mathrm{~B})$ & $11287(10)$ & $5590(4)$ & 1718(3) & 28(1) \\
\hline$C(4 B)$ & 11349(10) & 6754(4) & 2004(3) & 29(1) \\
\hline$C(5 B)$ & 11916(11) & $6725(4)$ & 2892(3) & $30(1)$ \\
\hline $\mathrm{C}(6 \mathrm{~B})$ & 11934(11) & $7722(4)$ & 3426(3) & $33(1)$ \\
\hline C(7B) & $10200(13)$ & 7864(5) & 3944(4) & $43(2)$ \\
\hline $\mathrm{C}(8 \mathrm{~B})$ & 10338(14) & 8806(5) & $4428(4)$ & $46(2)$ \\
\hline C(9B) & 12274(13) & $9573(5)$ & 4399(4) & $46(2)$ \\
\hline $\mathrm{C}(10 \mathrm{~B})$ & $14020(14)$ & $9445(5)$ & $3875(5)$ & $63(2)$ \\
\hline$C(11 B)$ & 13875(13) & $8504(5)$ & $3387(5)$ & $53(2)$ \\
\hline$C(12 B)$ & $7640(11)$ & $4427(4)$ & 2257(3) & 28(1) \\
\hline $\mathrm{O}(13 \mathrm{~B})$ & $7724(8)$ & $3519(3)$ & 1833(2) & $43(1)$ \\
\hline $\mathrm{O}(14 \mathrm{~B})$ & $5836(8)$ & 4701(3) & 2492(3) & $41(1)$ \\
\hline$C(15 B)$ & $5386(16)$ & $2847(7)$ & 1637(5) & $71(3)$ \\
\hline C(16B) & 4467(19) & 2964(8) & 836(5) & $85(3)$ \\
\hline$C(17 B)$ & $10005(12)$ & 5302(5) & $912(4)$ & $38(1)$ \\
\hline $\mathrm{O}(18 \mathrm{~B})$ & 7610(8) & 5607(3) & $865(2)$ & $41(1)$ \\
\hline C(19B) & $7657(12)$ & $6700(5)$ & 1003(3) & $38(1)$ \\
\hline C(20B) & 8921(14) & $7100(6)$ & 1794(4) & $47(2)$ \\
\hline $\mathrm{O}(21 \mathrm{~B})$ & 7889(15) & 7647(8) & 2211(3) & $132(4)$ \\
\hline $\mathrm{O}(22 \mathrm{~B})$ & 8891(7) & 7271(3) & 442(2) & $32(1)$ \\
\hline C(23B) & $7465(11)$ & $7328(4)$ & $-275(3)$ & 29(1) \\
\hline$C(24 B)$ & $8069(12)$ & 6529(4) & $-897(3)$ & $33(1)$ \\
\hline$C(25 B)$ & $6628(13)$ & $6578(5)$ & $-1684(3)$ & $39(2)$ \\
\hline C(26B) & 7145(15) & 7681(5) & $-1931(3)$ & $47(2)$ \\
\hline $\mathrm{C}(27 \mathrm{~B})$ & $6575(14)$ & $8492(5)$ & $-1300(3)$ & $40(2)$ \\
\hline$C(28 B)$ & $8004(11)$ & $8435(4)$ & $-523(3)$ & $32(1)$ \\
\hline C(29B) & 7292(16) & $5768(5)$ & $-2313(4)$ & $53(2)$ \\
\hline C(30B) & 7549(12) & $9269(5)$ & 129(4) & $38(1)$ \\
\hline$C(31 B)$ & 8558(17) & 10379(5) & $-87(5)$ & $61(2)$ \\
\hline C(32B) & 4918(15) & $9221(6)$ & 341(5) & $64(2)$ \\
\hline
\end{tabular}


Table S8. Bond lengths $[\AA]$ and angles $\left[{ }^{\circ}\right]$ for $\mathbf{2 b}$.

\begin{tabular}{|c|c|c|c|}
\hline $\mathrm{N}(1 \mathrm{~A})-\mathrm{C}(2 \mathrm{~A})$ & $1.454(6)$ & $\mathrm{N}(1 \mathrm{~A})-\mathrm{C}(5 \mathrm{~A})$ & $1.468(7)$ \\
\hline N(1A)-H(1A) & 0.8600 & $\mathrm{C}(2 \mathrm{~A})-\mathrm{C}(12 \mathrm{~A})$ & $1.504(8)$ \\
\hline C(2A)-C(3A) & $1.563(7)$ & $\mathrm{C}(2 \mathrm{~A})-\mathrm{H}(2 \mathrm{~A})$ & 0.9800 \\
\hline C(3A)-C(17A) & $1.511(8)$ & $C(3 A)-C(4 A)$ & $1.556(7)$ \\
\hline $\mathrm{C}(3 \mathrm{~A})-\mathrm{H}(3 \mathrm{~A})$ & 0.9800 & $C(4 A)-C(20 A)$ & $1.511(7)$ \\
\hline$C(4 A)-C(5 A)$ & $1.564(7)$ & $\mathrm{C}(4 \mathrm{~A})-\mathrm{H}(4 \mathrm{~A})$ & 0.9800 \\
\hline$C(5 A)-C(6 A)$ & $1.517(7)$ & $\mathrm{C}(5 \mathrm{~A})-\mathrm{H}(5 \mathrm{~A})$ & 0.9800 \\
\hline$C(6 A)-C(7 A)$ & $1.383(8)$ & $C(6 \mathrm{~A})-\mathrm{C}(11 \mathrm{~A})$ & $1.389(8)$ \\
\hline C(7A)-C(8A) & 1.391(7) & $\mathrm{C}(7 \mathrm{~A})-\mathrm{H}(7 \mathrm{~A})$ & 0.9300 \\
\hline C(8A)-C(9A) & 1.377(9) & $\mathrm{C}(8 \mathrm{~A})-\mathrm{H}(8 \mathrm{~A})$ & 0.9300 \\
\hline C(9A)-C(10A) & $1.375(10)$ & C(9A)-H(9A) & 0.9300 \\
\hline$C(10 A)-C(11 A)$ & $1.390(8)$ & $\mathrm{C}(10 \mathrm{~A})-\mathrm{H}(10 \mathrm{~A})$ & 0.9300 \\
\hline C(11A)-H(11A) & 0.9300 & $\mathrm{C}(12 \mathrm{~A})-\mathrm{O}(14 \mathrm{~A})$ & $1.184(7)$ \\
\hline$C(12 \mathrm{~A})-\mathrm{O}(13 \mathrm{~A})$ & $1.339(6)$ & $\mathrm{O}(13 \mathrm{~A})-\mathrm{C}(15 \mathrm{~A})$ & $1.468(8)$ \\
\hline$C(15 A)-C(16 A)$ & $1.358(12)$ & $\mathrm{C}(15 \mathrm{~A})-\mathrm{H}(15 \mathrm{~A})$ & 0.9700 \\
\hline $\mathrm{C}(15 \mathrm{~A})-\mathrm{H}(15 \mathrm{D})$ & 0.9700 & $\mathrm{C}(16 \mathrm{~A})-\mathrm{H}(16 \mathrm{~A})$ & 0.9600 \\
\hline C(16A)-H(16E) & 0.9600 & $\mathrm{C}(16 \mathrm{~A})-\mathrm{H}(16 \mathrm{~F})$ & 0.9600 \\
\hline C(17A)-O(18A) & $1.450(7)$ & $\mathrm{C}(17 \mathrm{~A})-\mathrm{H}(17 \mathrm{~A})$ & 0.9700 \\
\hline $\mathrm{C}(17 \mathrm{~A})-\mathrm{H}(17 \mathrm{D})$ & 0.9700 & $\mathrm{O}(18 \mathrm{~A})-\mathrm{C}(19 \mathrm{~A})$ & $1.418(7)$ \\
\hline $\mathrm{C}(19 \mathrm{~A})-\mathrm{O}(22 \mathrm{~A})$ & $1.395(6)$ & $C(19 A)-C(20 A)$ & $1.541(8)$ \\
\hline $\mathrm{C}(19 \mathrm{~A})-\mathrm{H}(19 \mathrm{~A})$ & 0.9800 & $C(20 A)-O(21 A)$ & $1.211(7)$ \\
\hline $\mathrm{O}(22 \mathrm{~A})-\mathrm{C}(23 \mathrm{~A})$ & $1.454(7)$ & $C(23 A)-C(28 A)$ & $1.524(7)$ \\
\hline$C(23 \mathrm{~A})-\mathrm{C}(24 \mathrm{~A})$ & $1.529(8)$ & $\mathrm{C}(23 \mathrm{~A})-\mathrm{H}(23 \mathrm{~A})$ & 0.9800 \\
\hline$C(24 \mathrm{~A})-\mathrm{C}(25 \mathrm{~A})$ & $1.535(9)$ & $\mathrm{C}(24 \mathrm{~A})-\mathrm{H}(24 \mathrm{~A})$ & 0.9700 \\
\hline $\mathrm{C}(24 \mathrm{~A})-\mathrm{H}(24 \mathrm{D})$ & 0.9700 & $C(25 A)-C(29 A)$ & $1.509(9)$ \\
\hline$C(25 A)-C(26 A)$ & $1.520(9)$ & $\mathrm{C}(25 \mathrm{~A})-\mathrm{H}(25 \mathrm{~A})$ & 0.9800 \\
\hline C(26A)-C(27A) & $1.524(8)$ & $\mathrm{C}(26 \mathrm{~A})-\mathrm{H}(26 \mathrm{~A})$ & 0.9700 \\
\hline $\mathrm{C}(26 \mathrm{~A})-\mathrm{H}(26 \mathrm{D})$ & 0.9700 & $C(27 A)-C(28 A)$ & $1.537(8)$ \\
\hline $\mathrm{C}(27 \mathrm{~A})-\mathrm{H}(27 \mathrm{~A})$ & 0.9700 & $\mathrm{C}(27 \mathrm{~A})-\mathrm{H}(27 \mathrm{D})$ & 0.9700 \\
\hline C(28A)-C(30A) & $1.530(8)$ & $\mathrm{C}(28 \mathrm{~A})-\mathrm{H}(28 \mathrm{~A})$ & 0.9800 \\
\hline C(29A)-H(29A) & 0.9600 & $\mathrm{C}(29 \mathrm{~A})-\mathrm{H}(29 \mathrm{E})$ & 0.9600 \\
\hline C(29A)-H(29F) & 0.9600 & $C(30 A)-C(32 A)$ & $1.527(9)$ \\
\hline$C(30 A)-C(31 A)$ & $1.527(8)$ & $\mathrm{C}(30 \mathrm{~A})-\mathrm{H}(30 \mathrm{~A})$ & 0.9800 \\
\hline $\mathrm{C}(31 \mathrm{~A})-\mathrm{H}(31 \mathrm{~A})$ & 0.9600 & $\mathrm{C}(31 \mathrm{~A})-\mathrm{H}(31 \mathrm{E})$ & 0.9600 \\
\hline $\mathrm{C}(31 \mathrm{~A})-\mathrm{H}(31 \mathrm{~F})$ & 0.9600 & $\mathrm{C}(32 \mathrm{~A})-\mathrm{H}(32 \mathrm{~A})$ & 0.9600 \\
\hline C(32A)-H(32E) & 0.9600 & $\mathrm{C}(32 \mathrm{~A})-\mathrm{H}(32 \mathrm{~F})$ & 0.9600 \\
\hline $\mathrm{N}(1 \mathrm{~B})-\mathrm{C}(2 \mathrm{~B})$ & $1.449(8)$ & $\mathrm{N}(1 \mathrm{~B})-\mathrm{C}(5 \mathrm{~B})$ & $1.465(7)$ \\
\hline $\mathrm{N}(1 \mathrm{~B})-\mathrm{H}(1 \mathrm{~B})$ & 0.8600 & $\mathrm{C}(2 \mathrm{~B})-\mathrm{C}(12 \mathrm{~B})$ & $1.519(8)$ \\
\hline C(2B)-C(3B) & $1.564(7)$ & C(2B)-H(2B) & 0.9800 \\
\hline C(3B)-C(17B) & $1.513(8)$ & C(3B)-C(4B) & $1.541(8)$ \\
\hline $\mathrm{C}(3 \mathrm{~B})-\mathrm{H}(3 \mathrm{~B})$ & 0.9800 & $\mathrm{C}(4 \mathrm{~B})-\mathrm{C}(20 \mathrm{~B})$ & $1.504(9)$ \\
\hline C(4B)-C(5B) & $1.545(8)$ & $\mathrm{C}(4 \mathrm{~B})-\mathrm{H}(4 \mathrm{~B})$ & 0.9800 \\
\hline C(5B)-C(6B) & $1.517(8)$ & C(5B)-H(5B) & 0.9800 \\
\hline C(6B)-C(7B) & $1.362(9)$ & $\mathrm{C}(6 \mathrm{~B})-\mathrm{C}(11 \mathrm{~B})$ & $1.380(8)$ \\
\hline C(7B)-C(8B) & $1.409(9)$ & $\mathrm{C}(7 \mathrm{~B})-\mathrm{H}(7 \mathrm{~B})$ & 0.9300 \\
\hline C(8B)-C(9B) & $1.363(9)$ & $\mathrm{C}(8 \mathrm{~B})-\mathrm{H}(8 \mathrm{~B})$ & 0.9300 \\
\hline C(9B)-C(10B) & $1.372(10)$ & C(9B)-H(9B) & 0.9300 \\
\hline $\mathrm{C}(10 \mathrm{~B})-\mathrm{C}(11 \mathrm{~B})$ & $1.410(9)$ & $\mathrm{C}(10 \mathrm{~B})-\mathrm{H}(10 \mathrm{~B})$ & 0.9300 \\
\hline C(11B)-H(11B) & 0.9300 & $\mathrm{C}(12 \mathrm{~B})-\mathrm{O}(14 \mathrm{~B})$ & $1.181(7)$ \\
\hline $\mathrm{C}(12 \mathrm{~B})-\mathrm{O}(13 \mathrm{~B})$ & $1.338(7)$ & $\mathrm{O}(13 \mathrm{~B})-\mathrm{C}(15 \mathrm{~B})$ & $1.462(8)$ \\
\hline $\mathrm{C}(15 \mathrm{~B})-\mathrm{C}(16 \mathrm{~B})$ & $1.467(11)$ & C(15B)-H(15B) & 0.9700 \\
\hline C(15B)-H(15C) & 0.9700 & C(16B)-H(16B) & 0.9600 \\
\hline C(16B)-H(16C) & 0.9600 & $\mathrm{C}(16 \mathrm{~B})-\mathrm{H}(16 \mathrm{D})$ & 0.9600 \\
\hline $\mathrm{C}(17 \mathrm{~B})-\mathrm{O}(18 \mathrm{~B})$ & $1.430(8)$ & $\mathrm{C}(17 \mathrm{~B})-\mathrm{H}(17 \mathrm{~B})$ & 0.9700 \\
\hline $\mathrm{C}(17 \mathrm{~B})-\mathrm{H}(17 \mathrm{C})$ & 0.9700 & $\mathrm{O}(18 \mathrm{~B})-\mathrm{C}(19 \mathrm{~B})$ & $1.411(8)$ \\
\hline C(19B)-O(22B) & $1.412(7)$ & C(19B)-C(20B) & $1.510(9)$ \\
\hline C(19B)-H(19B) & 0.9800 & $\mathrm{C}(20 \mathrm{~B})-\mathrm{O}(21 \mathrm{~B})$ & $1.187(8)$ \\
\hline $\mathrm{O}(22 \mathrm{~B})-\mathrm{C}(23 \mathrm{~B})$ & $1.438(6)$ & C(23B)-C(24B) & $1.506(8)$ \\
\hline C(23B)-C(28B) & $1.523(7)$ & C(23B)-H(23B) & 0.9800 \\
\hline $\mathrm{C}(24 \mathrm{~B})-\mathrm{C}(25 \mathrm{~B})$ & $1.541(8)$ & $\mathrm{C}(24 \mathrm{~B})-\mathrm{H}(24 \mathrm{~B})$ & 0.9700 \\
\hline C(24B)-H(24C) & 0.9700 & C(25B)-C(26B) & $1.518(8)$ \\
\hline$C(25 B)-C(29 B)$ & $1.536(9)$ & $\mathrm{C}(25 \mathrm{~B})-\mathrm{H}(25 \mathrm{~B})$ & 0.9800 \\
\hline $\mathrm{C}(26 \mathrm{~B})-\mathrm{C}(27 \mathrm{~B})$ & $1.520(9)$ & $\mathrm{C}(26 \mathrm{~B})-\mathrm{H}(26 \mathrm{~B})$ & 0.9700 \\
\hline $\mathrm{C}(26 \mathrm{~B})-\mathrm{H}(26 \mathrm{C})$ & 0.9700 & $\mathrm{C}(27 \mathrm{~B})-\mathrm{C}(28 \mathrm{~B})$ & $1.526(8)$ \\
\hline C(27B)-H(27B) & 0.9700 & C(27B)-H(27C) & 0.9700 \\
\hline C(28B)-C(30B) & $1.545(8)$ & $\mathrm{C}(28 \mathrm{~B})-\mathrm{H}(28 \mathrm{~B})$ & 0.9800 \\
\hline C(29B)-H(29B) & 0.9600 & C(29B)-H(29C) & 0.9600 \\
\hline
\end{tabular}




\begin{tabular}{|c|c|}
\hline C(29B)-H(29D) & 0.9600 \\
\hline C(30B)-C(31B) & $1.552(9)$ \\
\hline $\mathrm{C}(31 \mathrm{~B})-\mathrm{H}(31 \mathrm{~B})$ & 0.9600 \\
\hline C(31B)-H(31D) & 0.9600 \\
\hline $\mathrm{C}(32 \mathrm{~B})-\mathrm{H}(32 \mathrm{C})$ & 0.9600 \\
\hline $\mathrm{C}(2 \mathrm{~A})-\mathrm{N}(1 \mathrm{~A})-\mathrm{H}(1 \mathrm{~A})$ & 129.1 \\
\hline N(1A)-C(2A)-C(12A) & $112.2(4)$ \\
\hline$C(12 A)-C(2 A)-C(3 A)$ & $118.0(5)$ \\
\hline $\mathrm{C}(12 \mathrm{~A})-\mathrm{C}(2 \mathrm{~A})-\mathrm{H}(2 \mathrm{~A})$ & 107.2 \\
\hline$C(17 \mathrm{~A})-\mathrm{C}(3 \mathrm{~A})-\mathrm{C}(4 \mathrm{~A})$ & $111.3(4)$ \\
\hline $\mathrm{C}(4 \mathrm{~A})-\mathrm{C}(3 \mathrm{~A})-\mathrm{C}(2 \mathrm{~A})$ & 102.9(4) \\
\hline $\mathrm{C}(4 \mathrm{~A})-\mathrm{C}(3 \mathrm{~A})-\mathrm{H}(3 \mathrm{~A})$ & 109.3 \\
\hline$C(20 \mathrm{~A})-\mathrm{C}(4 \mathrm{~A})-\mathrm{C}(3 \mathrm{~A})$ & $113.8(4)$ \\
\hline C(3A)-C(4A)-C(5A) & 103.4(4) \\
\hline $\mathrm{C}(3 \mathrm{~A})-\mathrm{C}(4 \mathrm{~A})-\mathrm{H}(4 \mathrm{~A})$ & 108.6 \\
\hline$N(1 A)-C(5 A)-C(6 A)$ & $113.4(4)$ \\
\hline$C(6 A)-C(5 A)-C(4 A)$ & $119.0(4)$ \\
\hline C(6A)-C(5A)-H(5A) & 106.8 \\
\hline$C(7 A)-C(6 A)-C(11 A)$ & $118.6(5)$ \\
\hline$C(11 A)-C(6 A)-C(5 A)$ & 119.2(5) \\
\hline $\mathrm{C}(6 \mathrm{~A})-\mathrm{C}(7 \mathrm{~A})-\mathrm{H}(7 \mathrm{~A})$ & 119.6 \\
\hline$C(9 A)-C(8 A)-C(7 A)$ & 119.4(6) \\
\hline C(7A)-C(8A)-H(8A) & 120.3 \\
\hline $\mathrm{C}(10 \mathrm{~A})-\mathrm{C}(9 \mathrm{~A})-\mathrm{H}(9 \mathrm{~A})$ & 119.6 \\
\hline$C(9 A)-C(10 A)-C(11 A)$ & 119.3(6) \\
\hline $\mathrm{C}(11 \mathrm{~A})-\mathrm{C}(10 \mathrm{~A})-\mathrm{H}(10 \mathrm{~A})$ & 120.4 \\
\hline $\mathrm{C}(6 \mathrm{~A})-\mathrm{C}(11 \mathrm{~A})-\mathrm{H}(11 \mathrm{~A})$ & 119.5 \\
\hline$O(14 A)-C(12 A)-O(13 A)$ & 124.1(6) \\
\hline$O(13 \mathrm{~A})-\mathrm{C}(12 \mathrm{~A})-\mathrm{C}(2 \mathrm{~A})$ & $110.4(5)$ \\
\hline $\mathrm{C}(16 \mathrm{~A})-\mathrm{C}(15 \mathrm{~A})-\mathrm{O}(13 \mathrm{~A})$ & 109.8(7) \\
\hline $\mathrm{O}(13 \mathrm{~A})-\mathrm{C}(15 \mathrm{~A})-\mathrm{H}(15 \mathrm{~A})$ & 109.7 \\
\hline $\mathrm{O}(13 \mathrm{~A})-\mathrm{C}(15 \mathrm{~A})-\mathrm{H}(15 \mathrm{D})$ & 109.7 \\
\hline$C(15 A)-C(16 A)-H(16 A)$ & 109.5 \\
\hline $\mathrm{H}(16 \mathrm{~A})-\mathrm{C}(16 \mathrm{~A})-\mathrm{H}(16 \mathrm{E})$ & 109.5 \\
\hline $\mathrm{H}(16 \mathrm{~A})-\mathrm{C}(16 \mathrm{~A})-\mathrm{H}(16 \mathrm{~F})$ & 109.5 \\
\hline$O(18 A)-C(17 A)-C(3 A)$ & 109.5(4) \\
\hline C(3A)-C(17A)-H(17A) & 109.8 \\
\hline C(3A)-C(17A)-H(17D) & 109.8 \\
\hline $\mathrm{C}(19 \mathrm{~A})-\mathrm{O}(18 \mathrm{~A})-\mathrm{C}(17 \mathrm{~A})$ & 110.1(4) \\
\hline$O(22 A)-C(19 A)-C(20 A)$ & 107.2(4) \\
\hline $\mathrm{O}(22 \mathrm{~A})-\mathrm{C}(19 \mathrm{~A})-\mathrm{H}(19 \mathrm{~A})$ & 108.4 \\
\hline $\mathrm{C}(20 \mathrm{~A})-\mathrm{C}(19 \mathrm{~A})-\mathrm{H}(19 \mathrm{~A})$ & 108.4 \\
\hline$O(21 A)-C(20 A)-C(19 A)$ & $119.0(5)$ \\
\hline $\mathrm{C}(19 \mathrm{~A})-\mathrm{O}(22 \mathrm{~A})-\mathrm{C}(23 \mathrm{~A})$ & 114.1(4) \\
\hline$O(22 A)-C(23 A)-C(24 A)$ & 109.3(4) \\
\hline $\mathrm{O}(22 \mathrm{~A})-\mathrm{C}(23 \mathrm{~A})-\mathrm{H}(23 \mathrm{~A})$ & 109.2 \\
\hline $\mathrm{C}(24 \mathrm{~A})-\mathrm{C}(23 \mathrm{~A})-\mathrm{H}(23 \mathrm{~A})$ & 109.2 \\
\hline $\mathrm{C}(23 \mathrm{~A})-\mathrm{C}(24 \mathrm{~A})-\mathrm{H}(24 \mathrm{~A})$ & 109.3 \\
\hline $\mathrm{C}(23 \mathrm{~A})-\mathrm{C}(24 \mathrm{~A})-\mathrm{H}(24 \mathrm{D})$ & 109.3 \\
\hline $\mathrm{H}(24 \mathrm{~A})-\mathrm{C}(24 \mathrm{~A})-\mathrm{H}(24 \mathrm{D})$ & 108.0 \\
\hline$C(29 A)-C(25 A)-C(24 A)$ & $111.3(5)$ \\
\hline $\mathrm{C}(29 \mathrm{~A})-\mathrm{C}(25 \mathrm{~A})-\mathrm{H}(25 \mathrm{~A})$ & 108.3 \\
\hline $\mathrm{C}(24 \mathrm{~A})-\mathrm{C}(25 \mathrm{~A})-\mathrm{H}(25 \mathrm{~A})$ & 108.3 \\
\hline $\mathrm{C}(25 \mathrm{~A})-\mathrm{C}(26 \mathrm{~A})-\mathrm{H}(26 \mathrm{~A})$ & 109.2 \\
\hline $\mathrm{C}(25 \mathrm{~A})-\mathrm{C}(26 \mathrm{~A})-\mathrm{H}(26 \mathrm{D})$ & 109.2 \\
\hline$H(26 A)-C(26 A)-H(26 D)$ & 107.9 \\
\hline $\mathrm{C}(26 \mathrm{~A})-\mathrm{C}(27 \mathrm{~A})-\mathrm{H}(27 \mathrm{~A})$ & 109.2 \\
\hline $\mathrm{C}(26 \mathrm{~A})-\mathrm{C}(27 \mathrm{~A})-\mathrm{H}(27 \mathrm{D})$ & 109.2 \\
\hline $\mathrm{H}(27 \mathrm{~A})-\mathrm{C}(27 \mathrm{~A})-\mathrm{H}(27 \mathrm{D})$ & 107.9 \\
\hline$C(23 A)-C(28 A)-C(27 A)$ & $108.5(5)$ \\
\hline $\mathrm{C}(23 \mathrm{~A})-\mathrm{C}(28 \mathrm{~A})-\mathrm{H}(28 \mathrm{~A})$ & 106.9 \\
\hline $\mathrm{C}(27 \mathrm{~A})-\mathrm{C}(28 \mathrm{~A})-\mathrm{H}(28 \mathrm{~A})$ & 106.9 \\
\hline $\mathrm{C}(25 \mathrm{~A})-\mathrm{C}(29 \mathrm{~A})-\mathrm{H}(29 \mathrm{E})$ & 109.5 \\
\hline $\mathrm{C}(25 \mathrm{~A})-\mathrm{C}(29 \mathrm{~A})-\mathrm{H}(29 \mathrm{~F})$ & 109.5 \\
\hline H(29E)-C(29A)-H(29F) & 109.5 \\
\hline$C(32 A)-C(30 A)-C(28 A)$ & $113.0(5)$ \\
\hline $\mathrm{C}(32 \mathrm{~A})-\mathrm{C}(30 \mathrm{~A})-\mathrm{H}(30 \mathrm{~A})$ & 107.3 \\
\hline $\mathrm{C}(28 \mathrm{~A})-\mathrm{C}(30 \mathrm{~A})-\mathrm{H}(30 \mathrm{~A})$ & 107.3 \\
\hline $\mathrm{C}(30 \mathrm{~A})-\mathrm{C}(31 \mathrm{~A})-\mathrm{H}(31 \mathrm{E})$ & 109.5 \\
\hline
\end{tabular}

\begin{tabular}{|c|c|}
\hline C(30B)-C(32B) & $1.510(10$ \\
\hline C(30B)-H(30B) & 0.9800 \\
\hline C(31B)-H(31C) & 0.9600 \\
\hline $\mathrm{C}(32 \mathrm{~B})-\mathrm{H}(32 \mathrm{~B})$ & 0.9600 \\
\hline $\mathrm{C}(32 \mathrm{~B})-\mathrm{H}(32 \mathrm{D})$ & 0.9600 \\
\hline $\mathrm{C}(2 \mathrm{~A})-\mathrm{N}(1 \mathrm{~A})-\mathrm{C}(5 \mathrm{~A})$ & $101.8(4)$ \\
\hline $\mathrm{C}(5 \mathrm{~A})-\mathrm{N}(1 \mathrm{~A})-\mathrm{H}(1 \mathrm{~A})$ & 129.1 \\
\hline$N(1 A)-C(2 A)-C(3 A)$ & 104.6(4) \\
\hline $\mathrm{N}(1 \mathrm{~A})-\mathrm{C}(2 \mathrm{~A})-\mathrm{H}(2 \mathrm{~A})$ & 107.2 \\
\hline $\mathrm{C}(3 \mathrm{~A})-\mathrm{C}(2 \mathrm{~A})-\mathrm{H}(2 \mathrm{~A})$ & 107.2 \\
\hline $\mathrm{C}(17 \mathrm{~A})-\mathrm{C}(3 \mathrm{~A})-\mathrm{C}(2 \mathrm{~A})$ & $114.6(5)$ \\
\hline $\mathrm{C}(17 \mathrm{~A})-\mathrm{C}(3 \mathrm{~A})-\mathrm{H}(3 \mathrm{~A})$ & 109.3 \\
\hline $\mathrm{C}(2 \mathrm{~A})-\mathrm{C}(3 \mathrm{~A})-\mathrm{H}(3 \mathrm{~A})$ & 109.3 \\
\hline $\mathrm{C}(20 \mathrm{~A})-\mathrm{C}(4 \mathrm{~A})-\mathrm{C}(5 \mathrm{~A})$ & $113.7(4)$ \\
\hline $\mathrm{C}(20 \mathrm{~A})-\mathrm{C}(4 \mathrm{~A})-\mathrm{H}(4 \mathrm{~A})$ & 108.6 \\
\hline $\mathrm{C}(5 \mathrm{~A})-\mathrm{C}(4 \mathrm{~A})-\mathrm{H}(4 \mathrm{~A})$ & 108.6 \\
\hline$N(1 A)-C(5 A)-C(4 A)$ & 103.4(4) \\
\hline $\mathrm{N}(1 \mathrm{~A})-\mathrm{C}(5 \mathrm{~A})-\mathrm{H}(5 \mathrm{~A})$ & 106.8 \\
\hline $\mathrm{C}(4 \mathrm{~A})-\mathrm{C}(5 \mathrm{~A})-\mathrm{H}(5 \mathrm{~A})$ & 106.8 \\
\hline$C(7 A)-C(6 A)-C(5 A)$ & 122.3(5) \\
\hline$C(6 A)-C(7 A)-C(8 A)$ & $120.9(6)$ \\
\hline $\mathrm{C}(8 \mathrm{~A})-\mathrm{C}(7 \mathrm{~A})-\mathrm{H}(7 \mathrm{~A})$ & 119.6 \\
\hline $\mathrm{C}(9 \mathrm{~A})-\mathrm{C}(8 \mathrm{~A})-\mathrm{H}(8 \mathrm{~A})$ & 120.3 \\
\hline $\mathrm{C}(10 \mathrm{~A})-\mathrm{C}(9 \mathrm{~A})-\mathrm{C}(8 \mathrm{~A})$ & $120.9(5)$ \\
\hline $\mathrm{C}(8 \mathrm{~A})-\mathrm{C}(9 \mathrm{~A})-\mathrm{H}(9 \mathrm{~A})$ & 119.6 \\
\hline $\mathrm{C}(9 \mathrm{~A})-\mathrm{C}(10 \mathrm{~A})-\mathrm{H}(10 \mathrm{~A})$ & 120.4 \\
\hline$C(6 A)-C(11 A)-C(10 A)$ & $121.0(6)$ \\
\hline $\mathrm{C}(10 \mathrm{~A})-\mathrm{C}(11 \mathrm{~A})-\mathrm{H}(11 \mathrm{~A})$ & 119.5 \\
\hline$O(14 A)-C(12 A)-C(2 A)$ & 125.3(5) \\
\hline$C(12 A)-O(13 A)-C(15 A)$ & 115.4(5) \\
\hline $\mathrm{C}(16 \mathrm{~A})-\mathrm{C}(15 \mathrm{~A})-\mathrm{H}(15 \mathrm{~A})$ & 109.7 \\
\hline $\mathrm{C}(16 \mathrm{~A})-\mathrm{C}(15 \mathrm{~A})-\mathrm{H}(15 \mathrm{D})$ & 109.7 \\
\hline $\mathrm{H}(15 \mathrm{~A})-\mathrm{C}(15 \mathrm{~A})-\mathrm{H}(15 \mathrm{D})$ & 108.2 \\
\hline $\mathrm{C}(15 \mathrm{~A})-\mathrm{C}(16 \mathrm{~A})-\mathrm{H}(16 \mathrm{E})$ & 109.5 \\
\hline $\mathrm{C}(15 \mathrm{~A})-\mathrm{C}(16 \mathrm{~A})-\mathrm{H}(16 \mathrm{~F})$ & 109.5 \\
\hline $\mathrm{H}(16 \mathrm{E})-\mathrm{C}(16 \mathrm{~A})-\mathrm{H}(16 \mathrm{~F})$ & 109.5 \\
\hline $\mathrm{O}(18 \mathrm{~A})-\mathrm{C}(17 \mathrm{~A})-\mathrm{H}(17 \mathrm{~A})$ & 109.8 \\
\hline $\mathrm{O}(18 \mathrm{~A})-\mathrm{C}(17 \mathrm{~A})-\mathrm{H}(17 \mathrm{D})$ & 109.8 \\
\hline $\mathrm{H}(17 \mathrm{~A})-\mathrm{C}(17 \mathrm{~A})-\mathrm{H}(17 \mathrm{D})$ & 108.2 \\
\hline$O(22 A)-C(19 A)-O(18 A)$ & $112.2(5)$ \\
\hline $\mathrm{O}(18 \mathrm{~A})-\mathrm{C}(19 \mathrm{~A})-\mathrm{C}(20 \mathrm{~A})$ & $112.2(4)$ \\
\hline $\mathrm{O}(18 \mathrm{~A})-\mathrm{C}(19 \mathrm{~A})-\mathrm{H}(19 \mathrm{~A})$ & 108.4 \\
\hline$O(21 A)-C(20 A)-C(4 A)$ & 121.2(5) \\
\hline C(4A)-C(20A)-C(19A) & 119.8(5) \\
\hline$O(22 \mathrm{~A})-\mathrm{C}(23 \mathrm{~A})-\mathrm{C}(28 \mathrm{~A})$ & 108.5(4) \\
\hline$C(28 A)-C(23 A)-C(24 A)$ & $111.5(4)$ \\
\hline $\mathrm{C}(28 \mathrm{~A})-\mathrm{C}(23 \mathrm{~A})-\mathrm{H}(23 \mathrm{~A})$ & 109.2 \\
\hline$C(23 A)-C(24 A)-C(25 A)$ & 111.5(5) \\
\hline $\mathrm{C}(25 \mathrm{~A})-\mathrm{C}(24 \mathrm{~A})-\mathrm{H}(24 \mathrm{~A})$ & 109.3 \\
\hline $\mathrm{C}(25 \mathrm{~A})-\mathrm{C}(24 \mathrm{~A})-\mathrm{H}(24 \mathrm{D})$ & 109.3 \\
\hline$C(29 A)-C(25 A)-C(26 A)$ & $111.5(5)$ \\
\hline$C(26 A)-C(25 A)-C(24 A)$ & 109.2(5) \\
\hline $\mathrm{C}(26 \mathrm{~A})-\mathrm{C}(25 \mathrm{~A})-\mathrm{H}(25 \mathrm{~A})$ & 108.3 \\
\hline$C(25 A)-C(26 A)-C(27 A)$ & $111.9(5)$ \\
\hline $\mathrm{C}(27 \mathrm{~A})-\mathrm{C}(26 \mathrm{~A})-\mathrm{H}(26 \mathrm{~A})$ & 109.2 \\
\hline $\mathrm{C}(27 \mathrm{~A})-\mathrm{C}(26 \mathrm{~A})-\mathrm{H}(26 \mathrm{D})$ & 109.2 \\
\hline$C(26 A)-C(27 A)-C(28 A)$ & $112.0(5)$ \\
\hline $\mathrm{C}(28 \mathrm{~A})-\mathrm{C}(27 \mathrm{~A})-\mathrm{H}(27 \mathrm{~A})$ & 109.2 \\
\hline $\mathrm{C}(28 \mathrm{~A})-\mathrm{C}(27 \mathrm{~A})-\mathrm{H}(27 \mathrm{D})$ & 109.2 \\
\hline$C(23 A)-C(28 A)-C(30 A)$ & $113.5(5)$ \\
\hline$C(30 A)-C(28 A)-C(27 A)$ & 113.5(5) \\
\hline $\mathrm{C}(30 \mathrm{~A})-\mathrm{C}(28 \mathrm{~A})-\mathrm{H}(28 \mathrm{~A})$ & 106.9 \\
\hline $\mathrm{C}(25 \mathrm{~A})-\mathrm{C}(29 \mathrm{~A})-\mathrm{H}(29 \mathrm{~A})$ & 109.5 \\
\hline $\mathrm{H}(29 \mathrm{~A})-\mathrm{C}(29 \mathrm{~A})-\mathrm{H}(29 \mathrm{E})$ & 109.5 \\
\hline $\mathrm{H}(29 \mathrm{~A})-\mathrm{C}(29 \mathrm{~A})-\mathrm{H}(29 \mathrm{~F})$ & 109.5 \\
\hline$C(32 A)-C(30 A)-C(31 A)$ & $110.2(5)$ \\
\hline$C(31 A)-C(30 A)-C(28 A)$ & $111.5(5)$ \\
\hline $\mathrm{C}(31 \mathrm{~A})-\mathrm{C}(30 \mathrm{~A})-\mathrm{H}(30 \mathrm{~A})$ & 107.3 \\
\hline $\mathrm{C}(30 \mathrm{~A})-\mathrm{C}(31 \mathrm{~A})-\mathrm{H}(31 \mathrm{~A})$ & 109.5 \\
\hline $\mathrm{H}(31 \mathrm{~A})-\mathrm{C}(31 \mathrm{~A})-\mathrm{H}(31 \mathrm{E})$ & 109.5 \\
\hline
\end{tabular}




\begin{tabular}{|c|c|}
\hline $\mathrm{C}(30 \mathrm{~A})-\mathrm{C}(31 \mathrm{~A})-\mathrm{H}(31 \mathrm{~F})$ & 109.5 \\
\hline H(31E)-C(31A)-H(31F) & 109.5 \\
\hline $\mathrm{C}(30 \mathrm{~A})-\mathrm{C}(32 \mathrm{~A})-\mathrm{H}(32 \mathrm{E})$ & 109.5 \\
\hline $\mathrm{C}(30 \mathrm{~A})-\mathrm{C}(32 \mathrm{~A})-\mathrm{H}(32 \mathrm{~F})$ & 109.5 \\
\hline $\mathrm{H}(32 \mathrm{E})-\mathrm{C}(32 \mathrm{~A})-\mathrm{H}(32 \mathrm{~F})$ & 109.5 \\
\hline $\mathrm{C}(2 \mathrm{~B})-\mathrm{N}(1 \mathrm{~B})-\mathrm{H}(1 \mathrm{~B})$ & 126.7 \\
\hline N(1B)-C(2B)-C(12B) & 109.1(5) \\
\hline $\mathrm{C}(12 \mathrm{~B})-\mathrm{C}(2 \mathrm{~B})-\mathrm{C}(3 \mathrm{~B})$ & $115.4(4)$ \\
\hline $\mathrm{C}(12 \mathrm{~B})-\mathrm{C}(2 \mathrm{~B})-\mathrm{H}(2 \mathrm{~B})$ & 108.4 \\
\hline $\mathrm{C}(17 \mathrm{~B})-\mathrm{C}(3 \mathrm{~B})-\mathrm{C}(4 \mathrm{~B})$ & $113.0(5)$ \\
\hline $\mathrm{C}(4 \mathrm{~B})-\mathrm{C}(3 \mathrm{~B})-\mathrm{C}(2 \mathrm{~B})$ & 102.2(4) \\
\hline $\mathrm{C}(4 \mathrm{~B})-\mathrm{C}(3 \mathrm{~B})-\mathrm{H}(3 \mathrm{~B})$ & 107.7 \\
\hline $\mathrm{C}(20 \mathrm{~B})-\mathrm{C}(4 \mathrm{~B})-\mathrm{C}(3 \mathrm{~B})$ & $109.8(5)$ \\
\hline $\mathrm{C}(3 \mathrm{~B})-\mathrm{C}(4 \mathrm{~B})-\mathrm{C}(5 \mathrm{~B})$ & $100.7(4)$ \\
\hline $\mathrm{C}(3 \mathrm{~B})-\mathrm{C}(4 \mathrm{~B})-\mathrm{H}(4 \mathrm{~B})$ & 110.6 \\
\hline$N(1 B)-C(5 B)-C(6 B)$ & $116.0(5)$ \\
\hline$C(6 \mathrm{~B})-\mathrm{C}(5 \mathrm{~B})-\mathrm{C}(4 \mathrm{~B})$ & $118.6(4)$ \\
\hline C(6B)-C(5B)-H(5B) & 106.6 \\
\hline C(7B)-C(6B)-C(11B) & $119.5(6)$ \\
\hline$C(11 B)-C(6 B)-C(5 B)$ & $116.8(6)$ \\
\hline C(6B)-C(7B)-H(7B) & 119.6 \\
\hline $\mathrm{C}(9 \mathrm{~B})-\mathrm{C}(8 \mathrm{~B})-\mathrm{C}(7 \mathrm{~B})$ & $119.8(7)$ \\
\hline C(7B)-C(8B)-H(8B) & 120.1 \\
\hline C(8B)-C(9B)-H(9B) & 120.0 \\
\hline $\mathrm{C}(9 \mathrm{~B})-\mathrm{C}(10 \mathrm{~B})-\mathrm{C}(11 \mathrm{~B})$ & $120.2(6)$ \\
\hline $\mathrm{C}(11 \mathrm{~B})-\mathrm{C}(10 \mathrm{~B})-\mathrm{H}(10 \mathrm{~B})$ & 119.9 \\
\hline $\mathrm{C}(6 \mathrm{~B})-\mathrm{C}(11 \mathrm{~B})-\mathrm{H}(11 \mathrm{~B})$ & 120.1 \\
\hline $\mathrm{O}(14 \mathrm{~B})-\mathrm{C}(12 \mathrm{~B})-\mathrm{O}(13 \mathrm{~B})$ & $124.5(5)$ \\
\hline $\mathrm{O}(13 \mathrm{~B})-\mathrm{C}(12 \mathrm{~B})-\mathrm{C}(2 \mathrm{~B})$ & 110.1(5) \\
\hline O(13B)-C(15B)-C(16B) & $110.5(7)$ \\
\hline $\mathrm{C}(16 \mathrm{~B})-\mathrm{C}(15 \mathrm{~B})-\mathrm{H}(15 \mathrm{~B})$ & 109.5 \\
\hline C(16B)-C(15B)-H(15C) & 109.5 \\
\hline $\mathrm{C}(15 \mathrm{~B})-\mathrm{C}(16 \mathrm{~B})-\mathrm{H}(16 \mathrm{~B})$ & 109.5 \\
\hline $\mathrm{H}(16 \mathrm{~B})-\mathrm{C}(16 \mathrm{~B})-\mathrm{H}(16 \mathrm{C})$ & 109.5 \\
\hline $\mathrm{H}(16 \mathrm{~B})-\mathrm{C}(16 \mathrm{~B})-\mathrm{H}(16 \mathrm{D})$ & 109.5 \\
\hline $\mathrm{O}(18 \mathrm{~B})-\mathrm{C}(17 \mathrm{~B})-\mathrm{C}(3 \mathrm{~B})$ & 112.1(5) \\
\hline C(3B)-C(17B)-H(17B) & 109.2 \\
\hline C(3B)-C(17B)-H(17C) & 109.2 \\
\hline $\mathrm{C}(19 \mathrm{~B})-\mathrm{O}(18 \mathrm{~B})-\mathrm{C}(17 \mathrm{~B})$ & $112.5(5)$ \\
\hline O(18B)-C(19B)-C(20B) & $110.8(5)$ \\
\hline $\mathrm{O}(18 \mathrm{~B})-\mathrm{C}(19 \mathrm{~B})-\mathrm{H}(19 \mathrm{~B})$ & 108.5 \\
\hline C(20B)-C(19B)-H(19B) & 108.5 \\
\hline O(21B)-C(20B)-C(19B) & $117.2(7)$ \\
\hline C(19B)-O(22B)-C(23B) & 115.1(4) \\
\hline $\mathrm{O}(22 \mathrm{~B})-\mathrm{C}(23 \mathrm{~B})-\mathrm{C}(28 \mathrm{~B})$ & $108.5(4)$ \\
\hline $\mathrm{O}(22 \mathrm{~B})-\mathrm{C}(23 \mathrm{~B})-\mathrm{H}(23 \mathrm{~B})$ & 109.0 \\
\hline C(28B)-C(23B)-H(23B) & 109.0 \\
\hline C(23B)-C(24B)-H(24B) & 109.2 \\
\hline C(23B)-C(24B)-H(24C) & 109.2 \\
\hline $\mathrm{H}(24 \mathrm{~B})-\mathrm{C}(24 \mathrm{~B})-\mathrm{H}(24 \mathrm{C})$ & 107.9 \\
\hline$C(26 B)-C(25 B)-C(24 B)$ & $108.8(5)$ \\
\hline $\mathrm{C}(26 \mathrm{~B})-\mathrm{C}(25 \mathrm{~B})-\mathrm{H}(25 \mathrm{~B})$ & 108.5 \\
\hline C(24B)-C(25B)-H(25B) & 108.5 \\
\hline $\mathrm{C}(25 \mathrm{~B})-\mathrm{C}(26 \mathrm{~B})-\mathrm{H}(26 \mathrm{~B})$ & 109.3 \\
\hline C(25B)-C(26B)-H(26C) & 109.3 \\
\hline $\mathrm{H}(26 \mathrm{~B})-\mathrm{C}(26 \mathrm{~B})-\mathrm{H}(26 \mathrm{C})$ & 108.0 \\
\hline C(26B)-C(27B)-H(27B) & 109.2 \\
\hline C(26B)-C(27B)-H(27C) & 109.2 \\
\hline $\mathrm{H}(27 \mathrm{~B})-\mathrm{C}(27 \mathrm{~B})-\mathrm{H}(27 \mathrm{C})$ & 107.9 \\
\hline $\mathrm{C}(23 \mathrm{~B})-\mathrm{C}(28 \mathrm{~B})-\mathrm{C}(30 \mathrm{~B})$ & $112.0(5)$ \\
\hline C(23B)-C(28B)-H(28B) & 107.2 \\
\hline C(30B)-C(28B)-H(28B) & 107.2 \\
\hline C(25B)-C(29B)-H(29C) & 109.5 \\
\hline $\mathrm{C}(25 \mathrm{~B})-\mathrm{C}(29 \mathrm{~B})-\mathrm{H}(29 \mathrm{D})$ & 109.5 \\
\hline $\mathrm{H}(29 \mathrm{C})-\mathrm{C}(29 \mathrm{~B})-\mathrm{H}(29 \mathrm{D})$ & 109.5 \\
\hline C(32B)-C(30B)-C(31B) & $110.9(6)$ \\
\hline C(32B)-C(30B)-H(30B) & 106.9 \\
\hline C(31B)-C(30B)-H(30B) & 106.9 \\
\hline C(30B)-C(31B)-H(31C) & 109.5 \\
\hline $\mathrm{C}(30 \mathrm{~B})-\mathrm{C}(31 \mathrm{~B})-\mathrm{H}(31 \mathrm{D})$ & 109.5 \\
\hline
\end{tabular}

\begin{tabular}{|c|c|}
\hline $\mathrm{H}(31 \mathrm{~A})-\mathrm{C}(31 \mathrm{~A})-\mathrm{H}(31 \mathrm{~F})$ & 109.5 \\
\hline $\mathrm{C}(30 \mathrm{~A})-\mathrm{C}(32 \mathrm{~A})-\mathrm{H}(32 \mathrm{~A})$ & 109.5 \\
\hline $\mathrm{H}(32 \mathrm{~A})-\mathrm{C}(32 \mathrm{~A})-\mathrm{H}(32 \mathrm{E})$ & 109.5 \\
\hline $\mathrm{H}(32 \mathrm{~A})-\mathrm{C}(32 \mathrm{~A})-\mathrm{H}(32 \mathrm{~F})$ & 109.5 \\
\hline $\mathrm{C}(2 \mathrm{~B})-\mathrm{N}(1 \mathrm{~B})-\mathrm{C}(5 \mathrm{~B})$ & $106.5(5)$ \\
\hline $\mathrm{C}(5 \mathrm{~B})-\mathrm{N}(1 \mathrm{~B})-\mathrm{H}(1 \mathrm{~B})$ & 126.7 \\
\hline N(1B)-C(2B)-C(3B) & 107.1(4) \\
\hline $\mathrm{N}(1 \mathrm{~B})-\mathrm{C}(2 \mathrm{~B})-\mathrm{H}(2 \mathrm{~B})$ & 108.4 \\
\hline $\mathrm{C}(3 \mathrm{~B})-\mathrm{C}(2 \mathrm{~B})-\mathrm{H}(2 \mathrm{~B})$ & 108.4 \\
\hline C(17B)-C(3B)-C(2B) & 118.1(4) \\
\hline $\mathrm{C}(17 \mathrm{~B})-\mathrm{C}(3 \mathrm{~B})-\mathrm{H}(3 \mathrm{~B})$ & 107.7 \\
\hline $\mathrm{C}(2 \mathrm{~B})-\mathrm{C}(3 \mathrm{~B})-\mathrm{H}(3 \mathrm{~B})$ & 107.7 \\
\hline C(20B)-C(4B)-C(5B) & $114.2(5)$ \\
\hline C(20B)-C(4B)-H(4B) & 110.6 \\
\hline $\mathrm{C}(5 \mathrm{~B})-\mathrm{C}(4 \mathrm{~B})-\mathrm{H}(4 \mathrm{~B})$ & 110.6 \\
\hline N(1B)-C(5B)-C(4B) & $101.6(4)$ \\
\hline N(1B)-C(5B)-H(5B) & 106.6 \\
\hline C(4B)-C(5B)-H(5B) & 106.6 \\
\hline C(7B)-C(6B)-C(5B) & $123.7(5)$ \\
\hline $\mathrm{C}(6 \mathrm{~B})-\mathrm{C}(7 \mathrm{~B})-\mathrm{C}(8 \mathrm{~B})$ & $120.8(6)$ \\
\hline C(8B)-C(7B)-H(7B) & 119.6 \\
\hline $\mathrm{C}(9 \mathrm{~B})-\mathrm{C}(8 \mathrm{~B})-\mathrm{H}(8 \mathrm{~B})$ & 120.1 \\
\hline C(8B)-C(9B)-C(10B) & $120.0(6)$ \\
\hline C(10B)-C(9B)-H(9B) & 120.0 \\
\hline $\mathrm{C}(9 \mathrm{~B})-\mathrm{C}(10 \mathrm{~B})-\mathrm{H}(10 \mathrm{~B})$ & 119.9 \\
\hline C(6B)-C(11B)-C(10B) & $119.7(7)$ \\
\hline $\mathrm{C}(10 \mathrm{~B})-\mathrm{C}(11 \mathrm{~B})-\mathrm{H}(11 \mathrm{~B})$ & 120.1 \\
\hline $\mathrm{O}(14 \mathrm{~B})-\mathrm{C}(12 \mathrm{~B})-\mathrm{C}(2 \mathrm{~B})$ & $125.3(5)$ \\
\hline $\mathrm{C}(12 \mathrm{~B})-\mathrm{O}(13 \mathrm{~B})-\mathrm{C}(15 \mathrm{~B})$ & 116.5(6) \\
\hline $\mathrm{O}(13 \mathrm{~B})-\mathrm{C}(15 \mathrm{~B})-\mathrm{H}(15 \mathrm{~B})$ & 109.5 \\
\hline $\mathrm{O}(13 \mathrm{~B})-\mathrm{C}(15 \mathrm{~B})-\mathrm{H}(15 \mathrm{C})$ & 109.5 \\
\hline $\mathrm{H}(15 \mathrm{~B})-\mathrm{C}(15 \mathrm{~B})-\mathrm{H}(15 \mathrm{C})$ & 108.1 \\
\hline $\mathrm{C}(15 \mathrm{~B})-\mathrm{C}(16 \mathrm{~B})-\mathrm{H}(16 \mathrm{C})$ & 109.5 \\
\hline $\mathrm{C}(15 \mathrm{~B})-\mathrm{C}(16 \mathrm{~B})-\mathrm{H}(16 \mathrm{D})$ & 109.5 \\
\hline $\mathrm{H}(16 \mathrm{C})-\mathrm{C}(16 \mathrm{~B})-\mathrm{H}(16 \mathrm{D})$ & 109.5 \\
\hline $\mathrm{O}(18 \mathrm{~B})-\mathrm{C}(17 \mathrm{~B})-\mathrm{H}(17 \mathrm{~B})$ & 109.2 \\
\hline $\mathrm{O}(18 \mathrm{~B})-\mathrm{C}(17 \mathrm{~B})-\mathrm{H}(17 \mathrm{C})$ & 109.2 \\
\hline $\mathrm{H}(17 \mathrm{~B})-\mathrm{C}(17 \mathrm{~B})-\mathrm{H}(17 \mathrm{C})$ & 107.9 \\
\hline $\mathrm{O}(18 \mathrm{~B})-\mathrm{C}(19 \mathrm{~B})-\mathrm{O}(22 \mathrm{~B})$ & $113.5(5)$ \\
\hline $\mathrm{O}(22 \mathrm{~B})-\mathrm{C}(19 \mathrm{~B})-\mathrm{C}(20 \mathrm{~B})$ & $107.0(5)$ \\
\hline $\mathrm{O}(22 \mathrm{~B})-\mathrm{C}(19 \mathrm{~B})-\mathrm{H}(19 \mathrm{~B})$ & 108.5 \\
\hline $\mathrm{O}(21 \mathrm{~B})-\mathrm{C}(20 \mathrm{~B})-\mathrm{C}(4 \mathrm{~B})$ & $124.8(6)$ \\
\hline C(4B)-C(20B)-C(19B) & $117.9(6)$ \\
\hline $\mathrm{O}(22 \mathrm{~B})-\mathrm{C}(23 \mathrm{~B})-\mathrm{C}(24 \mathrm{~B})$ & $110.0(5)$ \\
\hline $\mathrm{C}(24 \mathrm{~B})-\mathrm{C}(23 \mathrm{~B})-\mathrm{C}(28 \mathrm{~B})$ & $111.3(4)$ \\
\hline $\mathrm{C}(24 \mathrm{~B})-\mathrm{C}(23 \mathrm{~B})-\mathrm{H}(23 \mathrm{~B})$ & 109.0 \\
\hline$C(23 B)-C(24 B)-C(25 B)$ & $112.0(5)$ \\
\hline $\mathrm{C}(25 \mathrm{~B})-\mathrm{C}(24 \mathrm{~B})-\mathrm{H}(24 \mathrm{~B})$ & 109.2 \\
\hline $\mathrm{C}(25 \mathrm{~B})-\mathrm{C}(24 \mathrm{~B})-\mathrm{H}(24 \mathrm{C})$ & 109.2 \\
\hline$C(26 B)-C(25 B)-C(29 B)$ & 111.2(5) \\
\hline$C(29 B)-C(25 B)-C(24 B)$ & $111.2(6)$ \\
\hline $\mathrm{C}(29 \mathrm{~B})-\mathrm{C}(25 \mathrm{~B})-\mathrm{H}(25 \mathrm{~B})$ & 108.5 \\
\hline $\mathrm{C}(25 \mathrm{~B})-\mathrm{C}(26 \mathrm{~B})-\mathrm{C}(27 \mathrm{~B})$ & $111.6(5)$ \\
\hline $\mathrm{C}(27 \mathrm{~B})-\mathrm{C}(26 \mathrm{~B})-\mathrm{H}(26 \mathrm{~B})$ & 109.3 \\
\hline $\mathrm{C}(27 \mathrm{~B})-\mathrm{C}(26 \mathrm{~B})-\mathrm{H}(26 \mathrm{C})$ & 109.3 \\
\hline$C(26 B)-C(27 B)-C(28 B)$ & 112.1(5) \\
\hline $\mathrm{C}(28 \mathrm{~B})-\mathrm{C}(27 \mathrm{~B})-\mathrm{H}(27 \mathrm{~B})$ & 109.2 \\
\hline $\mathrm{C}(28 \mathrm{~B})-\mathrm{C}(27 \mathrm{~B})-\mathrm{H}(27 \mathrm{C})$ & 109.2 \\
\hline C(23B)-C(28B)-C(27B) & $108.9(4)$ \\
\hline$C(27 \mathrm{~B})-\mathrm{C}(28 \mathrm{~B})-\mathrm{C}(30 \mathrm{~B})$ & $114.0(5)$ \\
\hline $\mathrm{C}(27 \mathrm{~B})-\mathrm{C}(28 \mathrm{~B})-\mathrm{H}(28 \mathrm{~B})$ & 107.2 \\
\hline $\mathrm{C}(25 \mathrm{~B})-\mathrm{C}(29 \mathrm{~B})-\mathrm{H}(29 \mathrm{~B})$ & 109.5 \\
\hline $\mathrm{H}(29 \mathrm{~B})-\mathrm{C}(29 \mathrm{~B})-\mathrm{H}(29 \mathrm{C})$ & 109.5 \\
\hline $\mathrm{H}(29 \mathrm{~B})-\mathrm{C}(29 \mathrm{~B})-\mathrm{H}(29 \mathrm{D})$ & 109.5 \\
\hline $\mathrm{C}(32 \mathrm{~B})-\mathrm{C}(30 \mathrm{~B})-\mathrm{C}(28 \mathrm{~B})$ & $114.8(5)$ \\
\hline $\mathrm{C}(28 \mathrm{~B})-\mathrm{C}(30 \mathrm{~B})-\mathrm{C}(31 \mathrm{~B})$ & 110.1(5) \\
\hline $\mathrm{C}(28 \mathrm{~B})-\mathrm{C}(30 \mathrm{~B})-\mathrm{H}(30 \mathrm{~B})$ & 106.9 \\
\hline $\mathrm{C}(30 \mathrm{~B})-\mathrm{C}(31 \mathrm{~B})-\mathrm{H}(31 \mathrm{~B})$ & 109.5 \\
\hline $\mathrm{H}(31 \mathrm{~B})-\mathrm{C}(31 \mathrm{~B})-\mathrm{H}(31 \mathrm{C})$ & 109.5 \\
\hline $\mathrm{H}(31 \mathrm{~B})-\mathrm{C}(31 \mathrm{~B})-\mathrm{H}(31 \mathrm{D})$ & 109.5 \\
\hline
\end{tabular}


$\mathrm{H}(31 \mathrm{C})-\mathrm{C}(31 \mathrm{~B})-\mathrm{H}(31 \mathrm{D})$

109.5

C(30B)-C(32B)-H(32C)

109.5

109.5

C(30B)-C(32B)-H(32B)

109.5

$\mathrm{C}(30 \mathrm{~B})-\mathrm{C}(32 \mathrm{~B})-\mathrm{H}(32 \mathrm{D})$

109.5

(32B)-C(32B)-H(32C)

H(32B)-C(32B)-H(32D)

109.5

109.5 
Table S9. Anisotropic displacement parameters $\left(\AA^{2} \times 10^{3}\right)$ for $\mathbf{2 b}$. The anisotropic displacement factor exponent takes the form: $-2 \pi^{2}\left[h^{2} a * 2 U^{11}+\ldots+2 h \mathrm{k} \mathrm{a}^{*} \mathrm{~b}^{*} \mathrm{U}^{12}\right]$.

\begin{tabular}{|c|c|c|c|c|c|c|}
\hline & $\mathrm{U}^{11}$ & $\mathrm{U}^{22}$ & $U^{33}$ & $\mathrm{U}^{23}$ & $\mathrm{U}^{13}$ & $\mathrm{U}^{12}$ \\
\hline $\mathrm{N}(1 \mathrm{~A})$ & 29(3) & $28(2)$ & $34(3)$ & $-5(2)$ & $10(2)$ & $-6(2)$ \\
\hline $\mathrm{C}(2 \mathrm{~A})$ & $25(3)$ & $32(3)$ & $20(3)$ & $2(2)$ & $3(2)$ & $-1(2)$ \\
\hline$C(3 A)$ & $27(3)$ & $26(3)$ & $24(3)$ & $2(2)$ & $2(2)$ & $-2(2)$ \\
\hline$C(4 A)$ & 23(3) & $25(2)$ & $18(2)$ & $2(2)$ & $1(2)$ & $3(2)$ \\
\hline$C(5 A)$ & $24(3)$ & $28(3)$ & 25(3) & $4(2)$ & $2(2)$ & $3(2)$ \\
\hline $\mathrm{C}(6 \mathrm{~A})$ & 31(3) & $28(3)$ & $24(3)$ & $4(2)$ & $6(2)$ & $1(2)$ \\
\hline$C(7 A)$ & $33(3)$ & $27(3)$ & 25(3) & $6(2)$ & $1(2)$ & $0(2)$ \\
\hline $\mathrm{C}(8 \mathrm{~A})$ & $43(4)$ & $31(3)$ & 23(3) & $1(2)$ & $5(2)$ & $-6(3)$ \\
\hline C(9A) & $56(5)$ & $32(3)$ & $41(4)$ & $-6(3)$ & 14(3) & $-1(3)$ \\
\hline$C(10 A)$ & $46(4)$ & $34(3)$ & $48(4)$ & $8(3)$ & $12(3)$ & $9(3)$ \\
\hline$C(11 \mathrm{~A})$ & $30(3)$ & $37(3)$ & $34(3)$ & $9(2)$ & $6(3)$ & $3(3)$ \\
\hline$C(12 A)$ & 31(3) & $37(3)$ & $20(3)$ & $0(2)$ & $-1(2)$ & $0(3)$ \\
\hline $\mathrm{O}(13 \mathrm{~A})$ & 39(3) & $44(2)$ & $56(3)$ & $-25(2)$ & $18(2)$ & $-8(2)$ \\
\hline$O(14 A)$ & $25(2)$ & $44(2)$ & $45(3)$ & $1(2)$ & $-2(2)$ & $1(2)$ \\
\hline$C(15 A)$ & $50(5)$ & $76(5)$ & $75(6)$ & $-37(5)$ & $22(4)$ & $7(4)$ \\
\hline $\mathrm{C}(16 \mathrm{~A})$ & 97(9) & $130(8)$ & 128(10) & $-56(8)$ & $-2(8)$ & 24(7) \\
\hline$C(17 A)$ & 38(3) & $29(3)$ & $27(3)$ & $3(2)$ & $2(2)$ & $6(3)$ \\
\hline $\mathrm{O}(18 \mathrm{~A})$ & $20(2)$ & $63(3)$ & $24(2)$ & $6(2)$ & $4(2)$ & $6(2)$ \\
\hline C(19A) & $28(3)$ & $33(3)$ & $20(3)$ & $2(2)$ & $-1(2)$ & $-4(2)$ \\
\hline$C(20 A)$ & $35(3)$ & $19(2)$ & $22(3)$ & $5(2)$ & $2(2)$ & $-9(2)$ \\
\hline$O(21 \mathrm{~A})$ & 49(3) & $35(2)$ & $24(2)$ & $0(2)$ & $6(2)$ & $8(2)$ \\
\hline $\mathrm{O}(22 \mathrm{~A})$ & $29(2)$ & $33(2)$ & $24(2)$ & $10(2)$ & $-1(2)$ & $4(2)$ \\
\hline$C(23 A)$ & $25(3)$ & 32(3) & $24(3)$ & $4(2)$ & $-2(2)$ & $3(2)$ \\
\hline$C(24 \mathrm{~A})$ & $37(4)$ & $39(3)$ & 29(3) & $4(2)$ & $3(3)$ & $6(3)$ \\
\hline$C(25 A)$ & $35(3)$ & $39(3)$ & $37(3)$ & $7(3)$ & 2(3) & $9(3)$ \\
\hline$C(26 A)$ & $40(4)$ & $39(3)$ & $40(3)$ & $7(3)$ & $-5(3)$ & $9(3)$ \\
\hline$C(27 A)$ & $45(4)$ & $40(3)$ & $32(3)$ & $1(3)$ & $-12(3)$ & 10(3) \\
\hline$C(28 \mathrm{~A})$ & $32(3)$ & $29(3)$ & $24(3)$ & $1(2)$ & $-1(2)$ & $2(2)$ \\
\hline$C(29 A)$ & $64(5)$ & $52(4)$ & $56(4)$ & $-5(3)$ & $-1(4)$ & $26(4)$ \\
\hline$C(30 A)$ & $39(4)$ & 34(3) & $29(3)$ & $-1(2)$ & $-5(3)$ & $5(3)$ \\
\hline$C(31 \mathrm{~A})$ & $47(4)$ & $52(4)$ & $31(3)$ & $-2(3)$ & $0(3)$ & 15(3) \\
\hline$C(32 A)$ & $62(5)$ & $33(3)$ & $54(4)$ & $-2(3)$ & $-2(4)$ & $-2(3)$ \\
\hline $\mathrm{N}(1 \mathrm{~B})$ & $74(4)$ & $42(3)$ & $22(3)$ & $4(2)$ & $6(3)$ & $-25(3)$ \\
\hline $\mathrm{C}(2 \mathrm{~B})$ & $30(3)$ & $33(3)$ & $31(3)$ & $10(2)$ & $-4(2)$ & $2(2)$ \\
\hline $\mathrm{C}(3 \mathrm{~B})$ & $24(3)$ & 31(3) & $31(3)$ & $13(2)$ & $4(2)$ & $3(2)$ \\
\hline $\mathrm{C}(4 \mathrm{~B})$ & 26(3) & $34(3)$ & $31(3)$ & $12(2)$ & $7(2)$ & $6(2)$ \\
\hline $\mathrm{C}(5 \mathrm{~B})$ & $25(3)$ & $28(3)$ & $37(3)$ & $9(2)$ & $-6(2)$ & $5(2)$ \\
\hline $\mathrm{C}(6 \mathrm{~B})$ & $25(3)$ & $35(3)$ & $36(3)$ & $10(2)$ & $-5(2)$ & $-3(2)$ \\
\hline $\mathrm{C}(7 \mathrm{~B})$ & $46(4)$ & $47(4)$ & 31(3) & $4(3)$ & $5(3)$ & $-13(3)$ \\
\hline $\mathrm{C}(8 \mathrm{~B})$ & $49(4)$ & $56(4)$ & $30(3)$ & $6(3)$ & $6(3)$ & $-3(3)$ \\
\hline C(9B) & $45(4)$ & $33(3)$ & $57(4)$ & $-4(3)$ & 1(3) & $3(3)$ \\
\hline C(10B) & $42(4)$ & $31(3)$ & $113(7)$ & $-8(4)$ & $18(5)$ & $-6(3)$ \\
\hline C(11B) & $32(4)$ & $39(3)$ & $85(5)$ & $-6(3)$ & $17(4)$ & $-2(3)$ \\
\hline $\mathrm{C}(12 \mathrm{~B})$ & 31(3) & 31(3) & 21(3) & $10(2)$ & $0(2)$ & $1(2)$ \\
\hline $\mathrm{O}(13 \mathrm{~B})$ & $47(3)$ & $39(2)$ & $37(2)$ & $-3(2)$ & $1(2)$ & $-9(2)$ \\
\hline $\mathrm{O}(14 \mathrm{~B})$ & 33(3) & $54(3)$ & $41(2)$ & $18(2)$ & $8(2)$ & $12(2)$ \\
\hline$C(15 B)$ & $71(6)$ & $80(6)$ & $45(4)$ & $-7(4)$ & $-2(4)$ & $-43(5)$ \\
\hline C(16B) & $86(7)$ & $114(8)$ & $47(5)$ & $15(5)$ & $-15(5)$ & $-13(6)$ \\
\hline C(17B) & $37(4)$ & 39(3) & $41(3)$ & 18(3) & $6(3)$ & $5(3)$ \\
\hline $\mathrm{O}(18 \mathrm{~B})$ & $32(2)$ & $53(3)$ & $41(2)$ & $26(2)$ & $-1(2)$ & $2(2)$ \\
\hline C(19B) & $32(3)$ & $58(4)$ & $30(3)$ & 19(3) & $10(3)$ & $18(3)$ \\
\hline C(20B) & $55(4)$ & $75(4)$ & $23(3)$ & $15(3)$ & $10(3)$ & $40(4)$ \\
\hline $\mathrm{O}(21 \mathrm{~B})$ & $136(7)$ & 238(9) & 43(3) & $-47(4)$ & $-33(4)$ & $158(7)$ \\
\hline $\mathrm{O}(22 \mathrm{~B})$ & $32(2)$ & $42(2)$ & $24(2)$ & $11(2)$ & $-1(2)$ & $7(2)$ \\
\hline C(23B) & $34(3)$ & $33(3)$ & $22(3)$ & $7(2)$ & $-3(2)$ & $8(2)$ \\
\hline$C(24 B)$ & $48(4)$ & $25(3)$ & $30(3)$ & $11(2)$ & 4(3) & $8(3)$ \\
\hline$C(25 B)$ & $53(4)$ & $35(3)$ & $27(3)$ & $3(2)$ & $1(3)$ & 2(3) \\
\hline$C(26 B)$ & $84(6)$ & $37(3)$ & $24(3)$ & $12(2)$ & $-6(3)$ & 16(3) \\
\hline$C(27 B)$ & $58(4)$ & $35(3)$ & $30(3)$ & $12(2)$ & $-3(3)$ & 14(3) \\
\hline $\mathrm{C}(28 \mathrm{~B})$ & $34(3)$ & $33(3)$ & $28(3)$ & $5(2)$ & $-4(2)$ & $4(2)$ \\
\hline$C(29 B)$ & $83(6)$ & $37(3)$ & $36(4)$ & $-5(3)$ & $2(4)$ & $6(4)$ \\
\hline$C(30 B)$ & $42(4)$ & $38(3)$ & $34(3)$ & $0(3)$ & $-1(3)$ & $6(3)$ \\
\hline
\end{tabular}


$\mathrm{C}(31 \mathrm{~B}) \quad 88(6)$

C(32B)
30(3)

61(5)
62(5)

69(5)
$-3(3)$

$-15(4)$
$-6(4)$

11(4)
8(4)

14(4) 
Table S10. Hydrogen coordinates $\left(\times 10^{4}\right)$ and isotropic displacement parameters $\left(\AA^{2} \times 10^{3}\right)$ for $\mathbf{2 b}$.

\begin{tabular}{|c|c|c|c|c|}
\hline & $\mathrm{x}$ & $\mathrm{y}$ & $\mathrm{z}$ & $\mathrm{U}(\mathrm{eq})$ \\
\hline $\mathrm{H}(1 \mathrm{~A})$ & 9982 & 4366 & 4190 & 38 \\
\hline $\mathrm{H}(2 \mathrm{~A})$ & 6400 & 3053 & 3406 & 32 \\
\hline $\mathrm{H}(3 \mathrm{~A})$ & 4761 & 1962 & 4233 & 32 \\
\hline $\mathrm{H}(4 \mathrm{~A})$ & 3612 & 3263 & 4956 & 26 \\
\hline $\mathrm{H}(5 \mathrm{~A})$ & 5142 & 4367 & 4126 & 31 \\
\hline $\mathrm{H}(7 \mathrm{~A})$ & 10057 & 5255 & 5569 & 34 \\
\hline $\mathrm{H}(8 \mathrm{~A})$ & 10715 & 6915 & 6232 & 40 \\
\hline $\mathrm{H}(9 \mathrm{~A})$ & 7772 & 8010 & 6124 & 53 \\
\hline $\mathrm{H}(10 \mathrm{~A})$ & 4333 & 7523 & 5285 & 50 \\
\hline $\mathrm{H}(11 \mathrm{~A})$ & 3715 & 5875 & 4605 & 40 \\
\hline $\mathrm{H}(15 \mathrm{~A})$ & 10937 & 1361 & 2261 & 83 \\
\hline $\mathrm{H}(15 \mathrm{D})$ & 11796 & 845 & 3007 & 83 \\
\hline $\mathrm{H}(16 \mathrm{~A})$ & 8836 & -448 & 2772 & 146 \\
\hline $\mathrm{H}(16 \mathrm{E})$ & 10266 & -325 & 2014 & 146 \\
\hline $\mathrm{H}(16 \mathrm{~F})$ & 7732 & 86 & 2075 & 146 \\
\hline $\mathrm{H}(17 \mathrm{~A})$ & 6691 & 1405 & 5312 & 38 \\
\hline $\mathrm{H}(17 \mathrm{D})$ & 8413 & 1277 & 4621 & 38 \\
\hline $\mathrm{H}(19 \mathrm{~A})$ & 9956 & 3602 & 6264 & 33 \\
\hline $\mathrm{H}(23 \mathrm{~A})$ & 11883 & 2518 & 6917 & 32 \\
\hline $\mathrm{H}(24 \mathrm{~A})$ & 10866 & 916 & 6143 & 42 \\
\hline $\mathrm{H}(24 \mathrm{D})$ & 9229 & 460 & 6787 & 42 \\
\hline $\mathrm{H}(25 \mathrm{~A})$ & 14405 & 999 & 6982 & 44 \\
\hline $\mathrm{H}(26 \mathrm{~A})$ & 14186 & 407 & 8225 & 47 \\
\hline $\mathrm{H}(26 \mathrm{D})$ & 11330 & 129 & 8097 & 47 \\
\hline $\mathrm{H}(27 \mathrm{~A})$ & 13903 & 2190 & 8221 & 48 \\
\hline $\mathrm{H}(27 \mathrm{D})$ & 12268 & 1726 & 8862 & 48 \\
\hline $\mathrm{H}(28 \mathrm{~A})$ & 8761 & 1611 & 8006 & 34 \\
\hline $\mathrm{H}(29 \mathrm{~A})$ & 11806 & -1036 & 6915 & 84 \\
\hline $\mathrm{H}(29 \mathrm{E})$ & 14667 & -759 & 6986 & 84 \\
\hline $\mathrm{H}(29 \mathrm{~F})$ & 13182 & -594 & 6212 & 84 \\
\hline $\mathrm{H}(30 \mathrm{~A})$ & 8411 & 3396 & 7942 & 41 \\
\hline $\mathrm{H}(31 \mathrm{~A})$ & 10284 & 3015 & 9436 & 65 \\
\hline $\mathrm{H}(31 \mathrm{E})$ & 7590 & 2607 & 9107 & 65 \\
\hline $\mathrm{H}(31 \mathrm{~F})$ & 8460 & 3814 & 9271 & 65 \\
\hline $\mathrm{H}(32 \mathrm{~A})$ & 12494 & 4027 & 7715 & 76 \\
\hline H(32E) & 13282 & 3879 & 8584 & 76 \\
\hline $\mathrm{H}(32 \mathrm{~F})$ & 11532 & 4700 & 8403 & 76 \\
\hline $\mathrm{H}(1 \mathrm{~B})$ & 9331 & 5776 & 3464 & 58 \\
\hline $\mathrm{H}(2 \mathrm{~B})$ & 11305 & 4535 & 2576 & 38 \\
\hline $\mathrm{H}(3 \mathrm{~B})$ & 12985 & 5454 & 1688 & 34 \\
\hline $\mathrm{H}(4 \mathrm{~B})$ & 12678 & 7186 & 1776 & 35 \\
\hline $\mathrm{H}(5 \mathrm{~B})$ & 13557 & 6522 & 2955 & 36 \\
\hline H(7B) & 8908 & 7332 & 3978 & 51 \\
\hline $\mathrm{H}(8 \mathrm{~B})$ & 9113 & 8905 & 4766 & 55 \\
\hline H(9B) & 12411 & 10183 & 4735 & 55 \\
\hline $\mathrm{H}(10 \mathrm{~B})$ & 15303 & 9981 & 3840 & 76 \\
\hline $\mathrm{H}(11 \mathrm{~B})$ & 15083 & 8410 & 3042 & 63 \\
\hline $\mathrm{H}(15 \mathrm{~B})$ & 4199 & 3032 & 2001 & 85 \\
\hline $\mathrm{H}(15 \mathrm{C})$ & 5599 & 2122 & 1683 & 85 \\
\hline $\mathrm{H}(16 \mathrm{~B})$ & 5547 & 2703 & 471 & 128 \\
\hline $\mathrm{H}(16 \mathrm{C})$ & 4398 & 3691 & 777 & 128 \\
\hline $\mathrm{H}(16 \mathrm{D})$ & 2857 & 2576 & 735 & 128 \\
\hline $\mathrm{H}(17 \mathrm{~B})$ & 10980 & 5641 & 528 & 46 \\
\hline $\mathrm{H}(17 \mathrm{C})$ & 9877 & 4551 & 787 & 46 \\
\hline $\mathrm{H}(19 \mathrm{~B})$ & 5965 & 6852 & 998 & 45 \\
\hline $\mathrm{H}(23 \mathrm{~B})$ & 5722 & 7180 & -182 & 35 \\
\hline $\mathrm{H}(24 \mathrm{~B})$ & 7692 & 5834 & -723 & 40 \\
\hline $\mathrm{H}(24 \mathrm{C})$ & 9811 & 6651 & -970 & 40 \\
\hline $\mathrm{H}(25 \mathrm{~B})$ & 4875 & 6422 & -1608 & 47 \\
\hline $\mathrm{H}(26 \mathrm{~B})$ & 6160 & 7730 & -2408 & 56 \\
\hline $\mathrm{H}(26 \mathrm{C})$ & 8855 & 7828 & -2041 & 56 \\
\hline $\mathrm{H}(27 \mathrm{~B})$ & 4835 & 8382 & -1224 & 48 \\
\hline $\mathrm{H}(27 \mathrm{C})$ & 6975 & 9186 & -1473 & 48 \\
\hline
\end{tabular}




\begin{tabular}{lrrrr}
$H(28 B)$ & 9750 & 8558 & -617 & 38 \\
$\mathrm{H}(29 B)$ & 9006 & 5911 & -2395 & 80 \\
$\mathrm{H}(29 \mathrm{C})$ & 6352 & 5808 & -2794 & 80 \\
$\mathrm{H}(29 \mathrm{D})$ & 6932 & 5078 & -2144 & 80 \\
$\mathrm{H}(30 \mathrm{~B})$ & 8509 & 9145 & 601 & 46 \\
$\mathrm{H}(31 \mathrm{~B})$ & 7650 & 10534 & -546 & 92 \\
$\mathrm{H}(31 \mathrm{C})$ & 10256 & 10404 & -190 & 92 \\
$\mathrm{H}(31 \mathrm{D})$ & 8393 & 10887 & 340 & 92 \\
$\mathrm{H}(32 \mathrm{~B})$ & 4333 & 8529 & 473 & 96 \\
$\mathrm{H}(32 \mathrm{C})$ & 3931 & 9385 & -97 & 96 \\
$\mathrm{H}(32 \mathrm{D})$ & 4820 & 9718 & 781 & 96 \\
\hline
\end{tabular}


${ }^{1} \mathrm{H} \mathrm{NMR}\left(\mathrm{CDCl}_{3}, 500 \mathrm{MHz}\right)$ of compound 3a
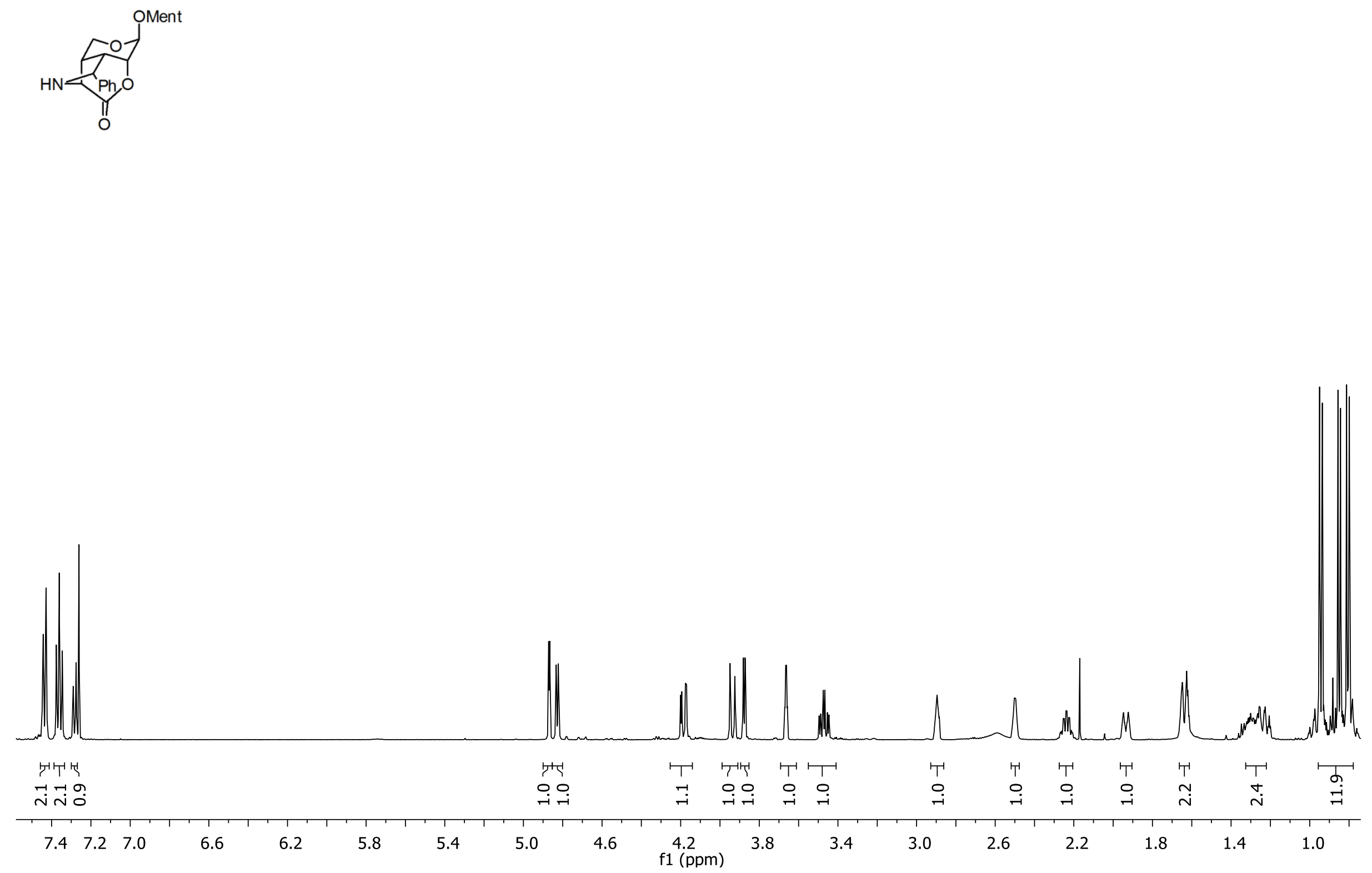
${ }^{13} \mathrm{C} \mathrm{NMR}\left(\mathrm{CDCl}_{3}, 125.7 \mathrm{MHz}\right)$ of compound 3a

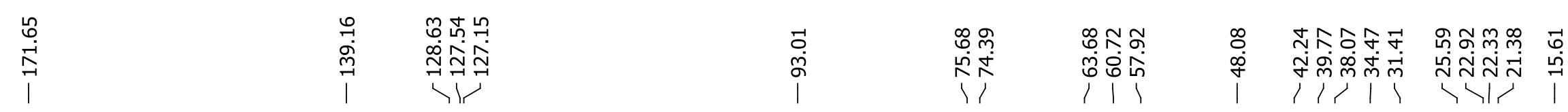

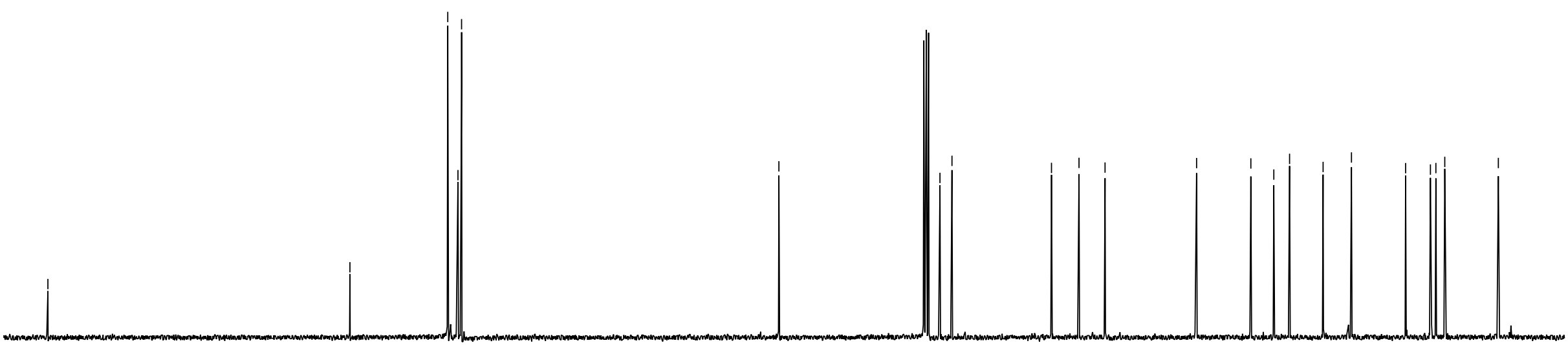




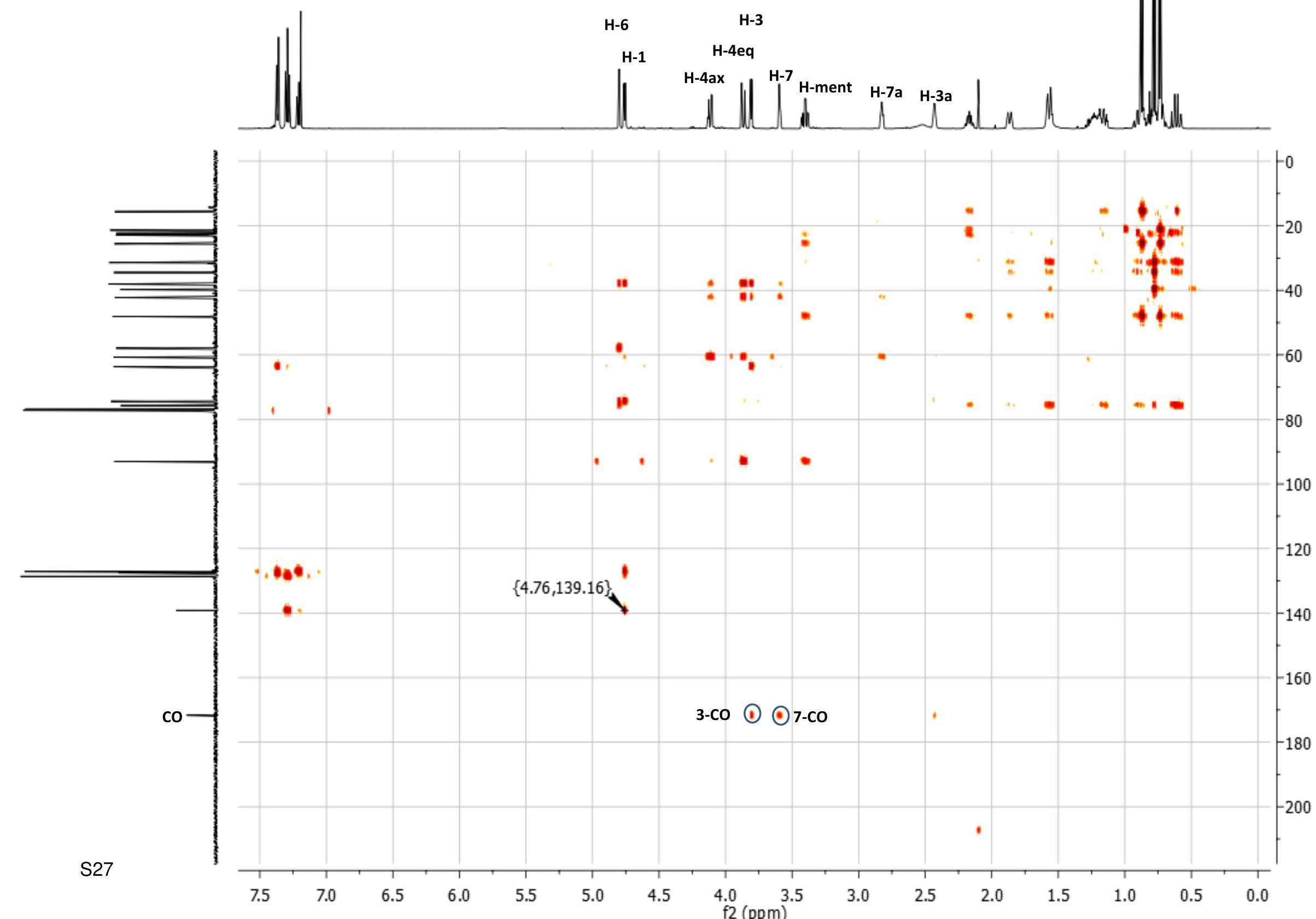


${ }^{1} \mathrm{H} \mathrm{NMR}\left(\mathrm{CDCl}_{3}, 500 \mathrm{MHz}\right)$ of compound 4a
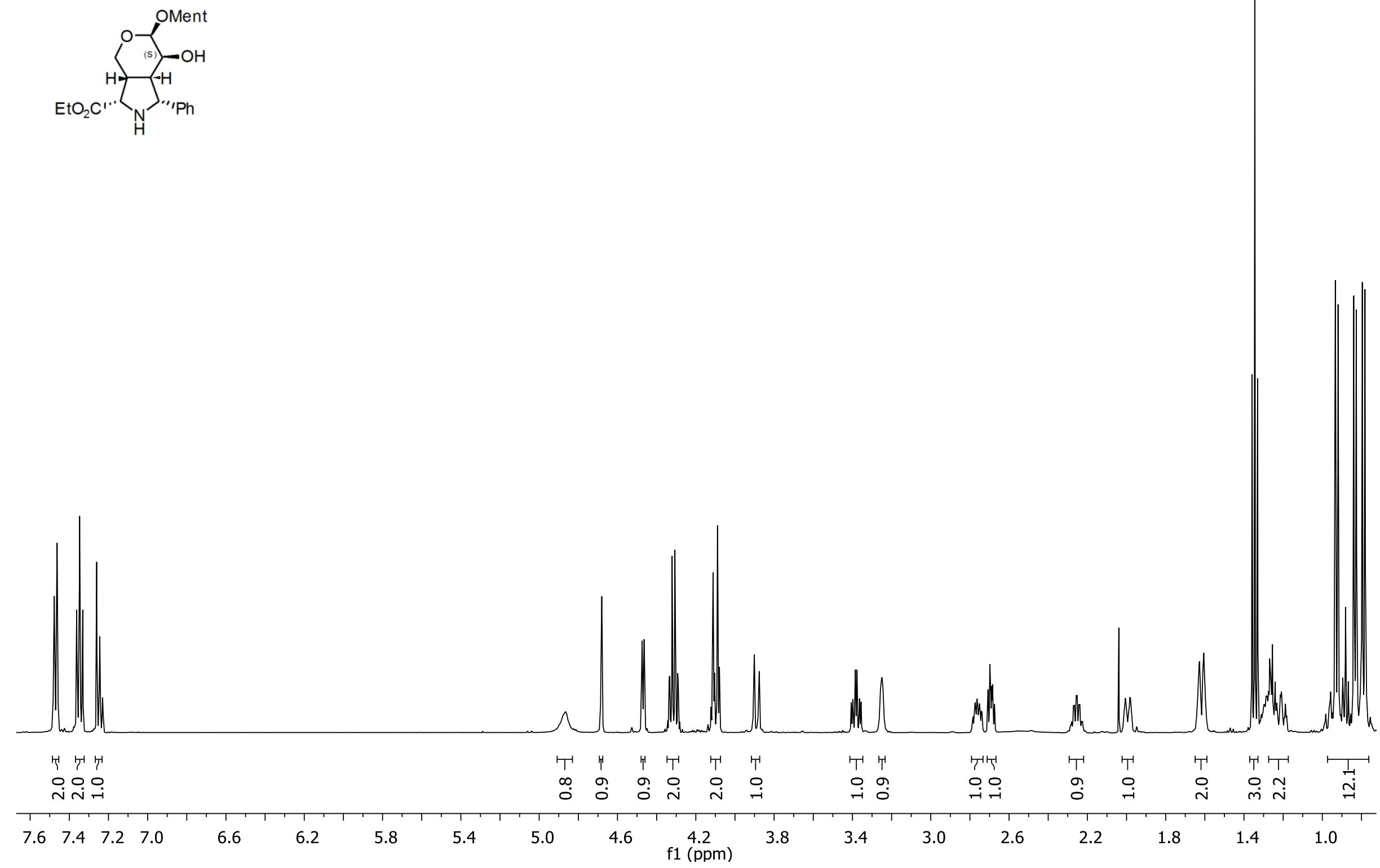
${ }^{13} \mathrm{C} \mathrm{NMR}\left(\mathrm{CDCl}_{3}, 125.7 \mathrm{MHz}\right)$ of compound 4a

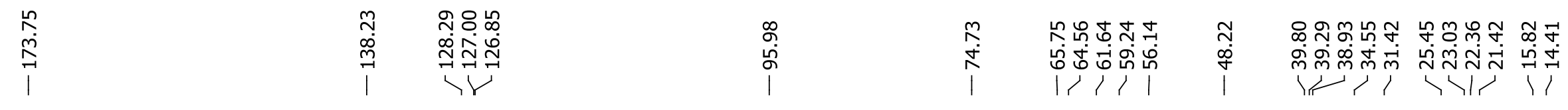

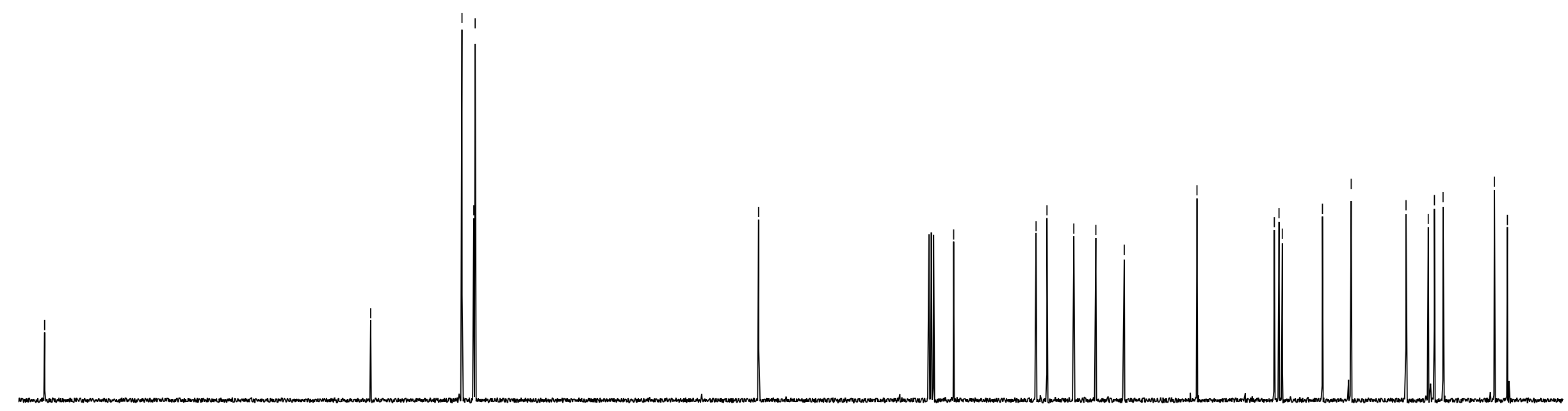

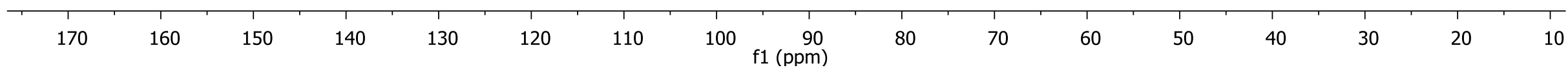




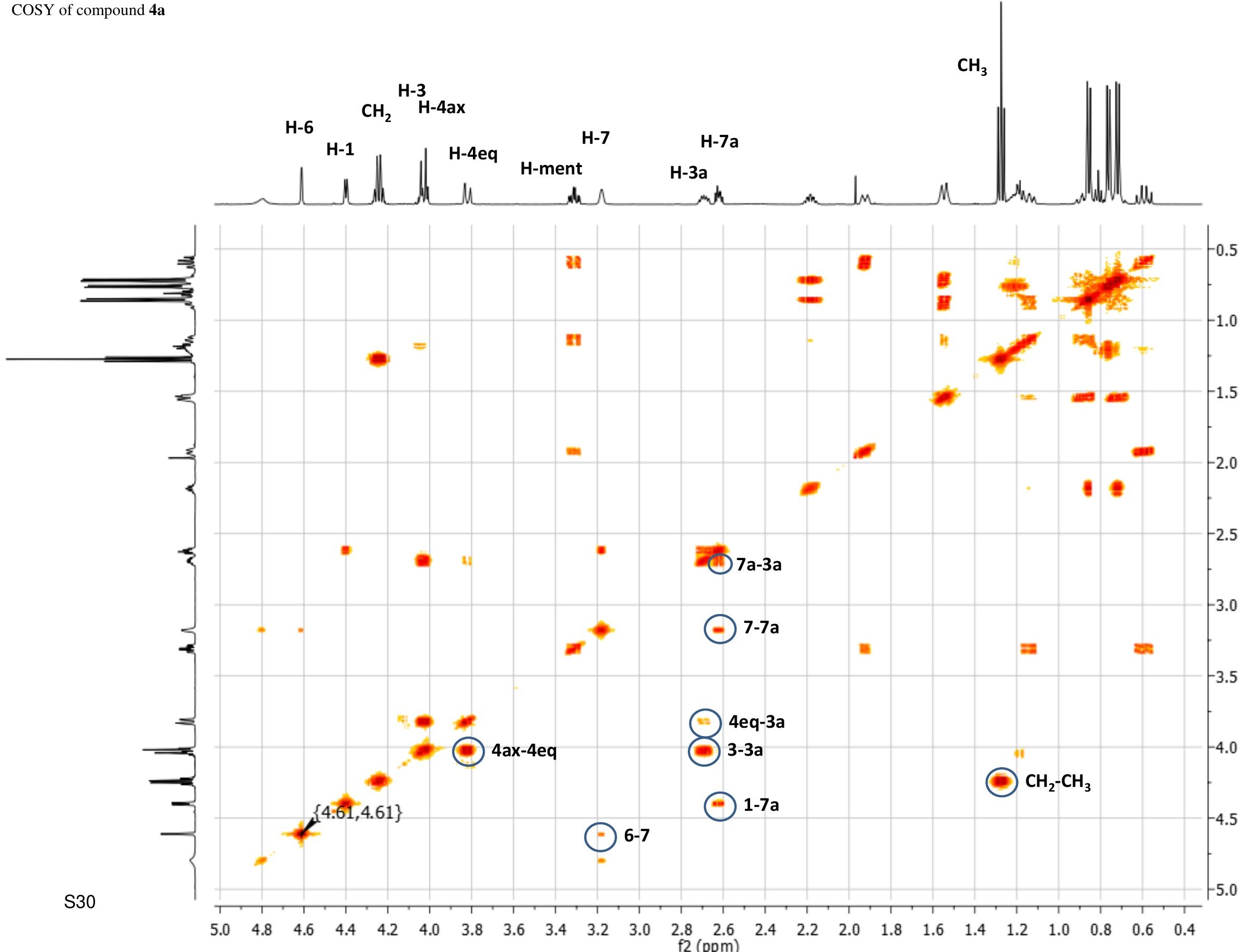


H-3

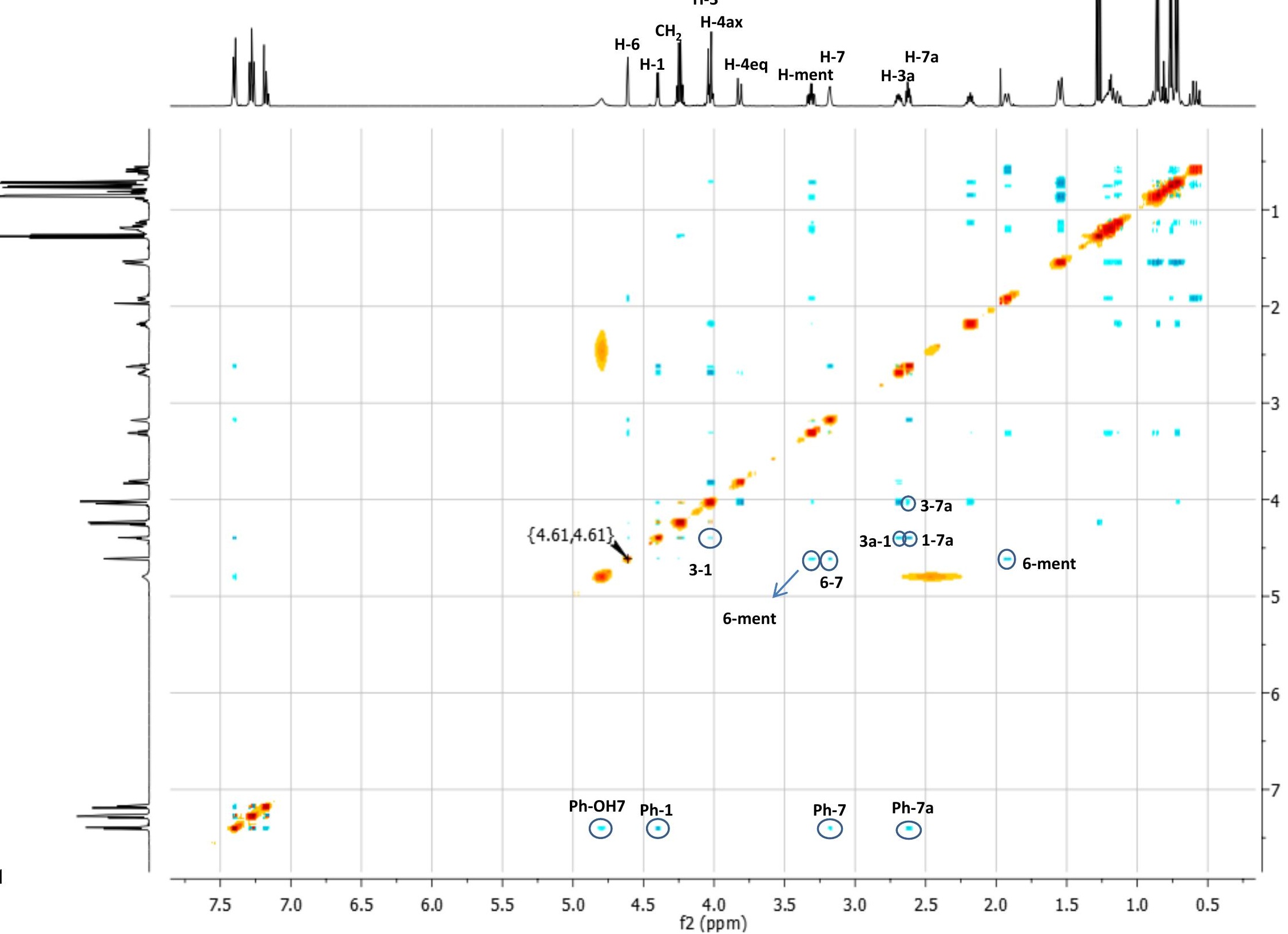


${ }^{1} \mathrm{H} \mathrm{NMR}\left(\mathrm{CDCl}_{3}, 500 \mathrm{MHz}\right)$ of compound $\mathbf{3 b}$
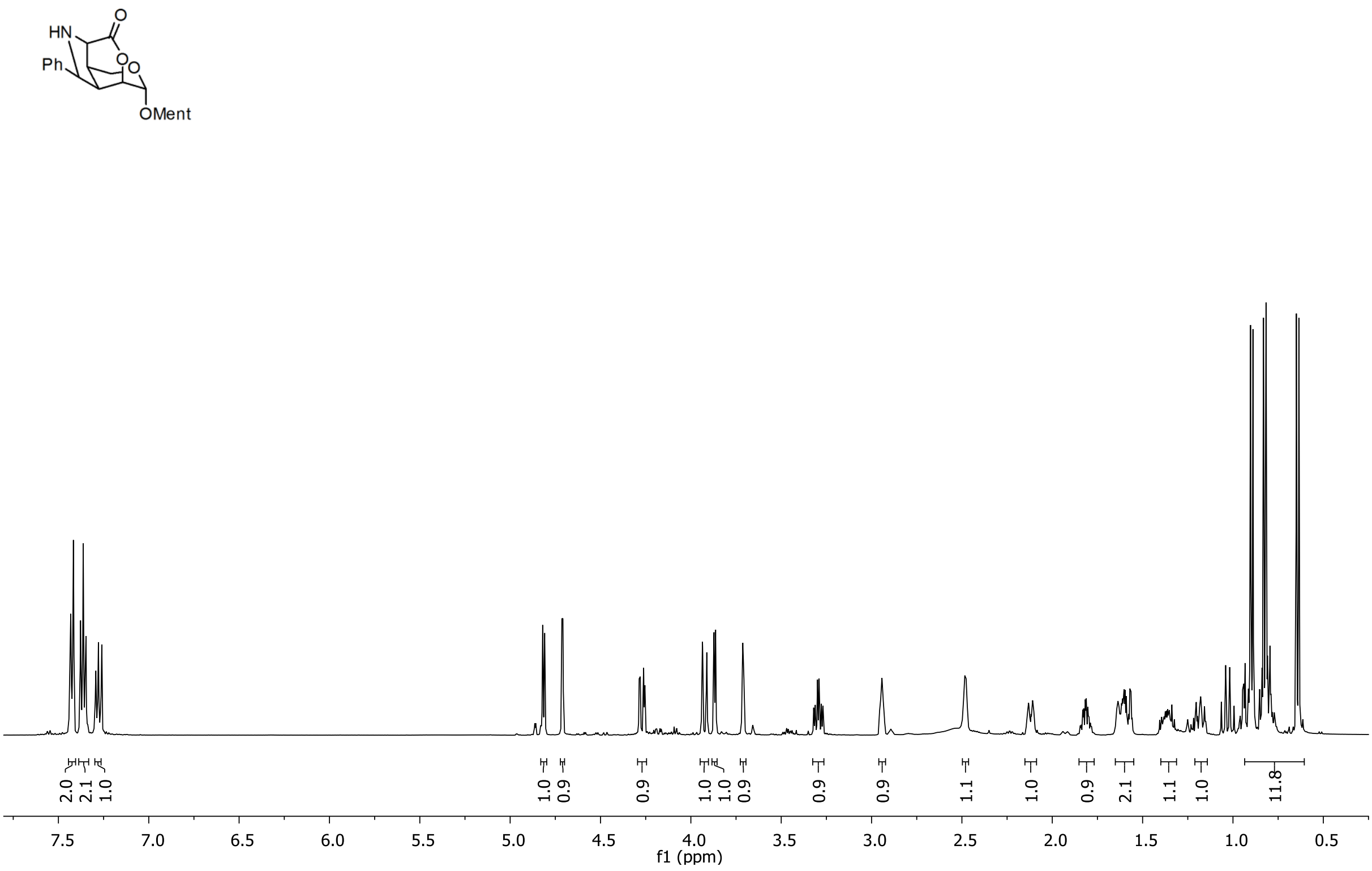
${ }^{13} \mathrm{C} \mathrm{NMR}\left(\mathrm{CDCl}_{3}, 125.7 \mathrm{MHz}\right)$ of compound $\mathbf{3 b}$

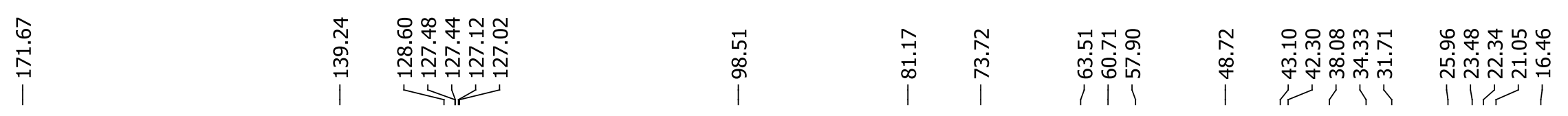

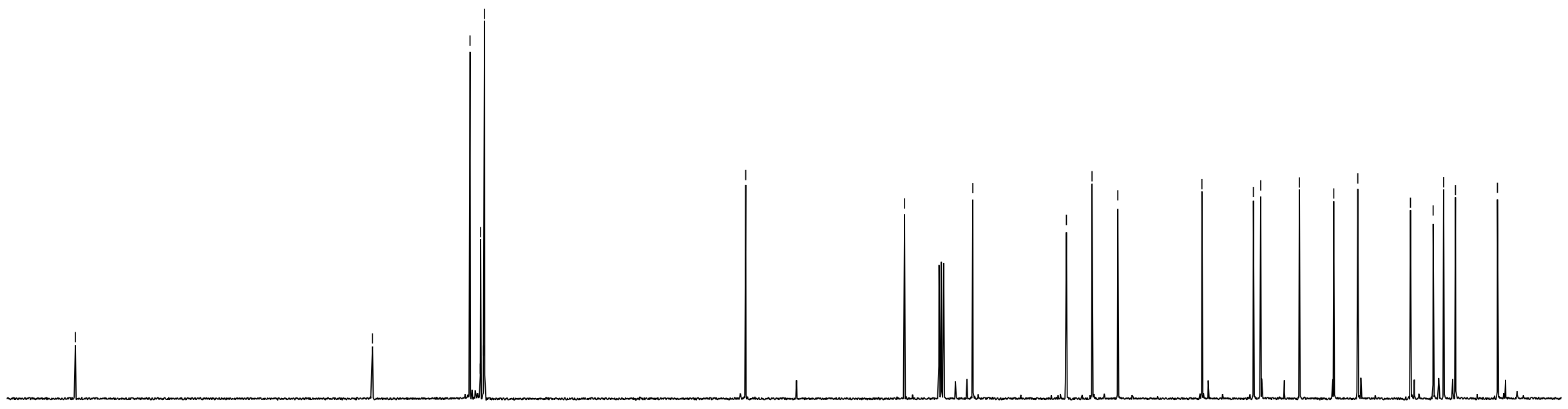

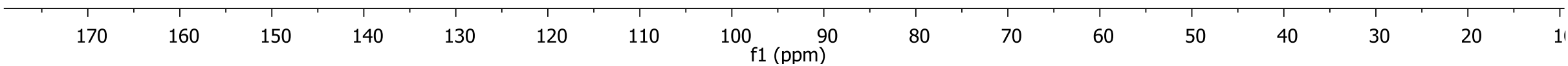


${ }^{1} \mathrm{H} \mathrm{NMR}\left(\mathrm{CDCl}_{3}, 500 \mathrm{MHz}\right)$ of compound $\mathbf{4 b}$
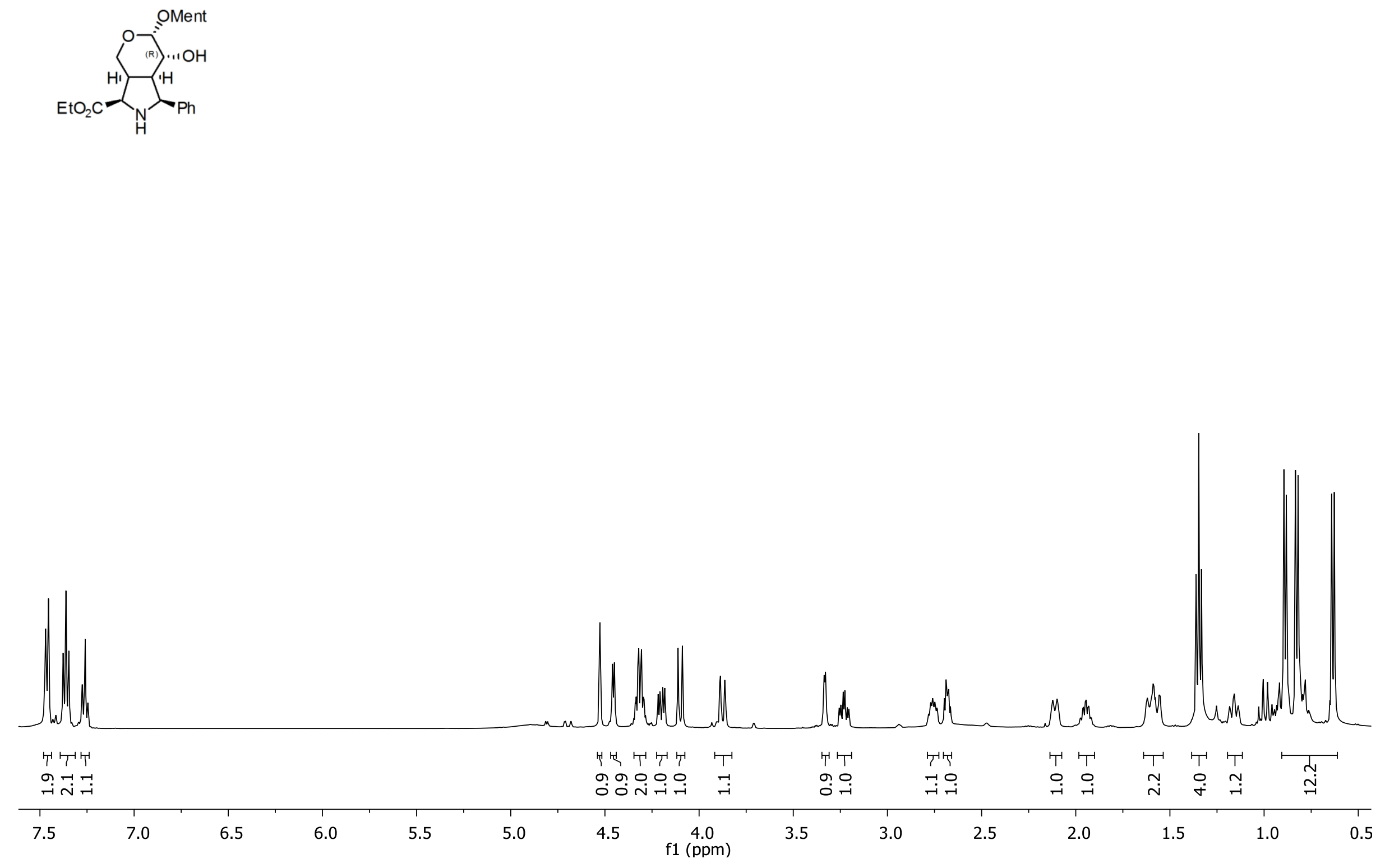
${ }^{13} \mathrm{C} \mathrm{NMR}\left(\mathrm{CDCl}_{3}, 125.7 \mathrm{MHz}\right)$ of compound $\mathbf{4 b}$

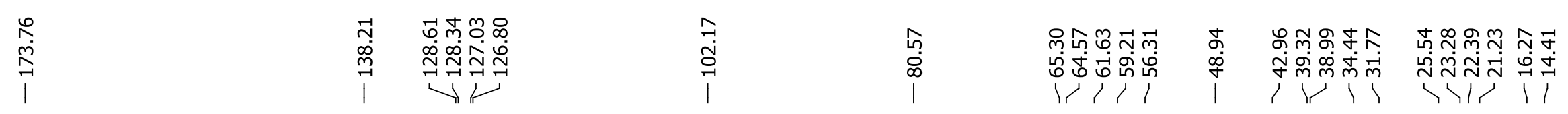

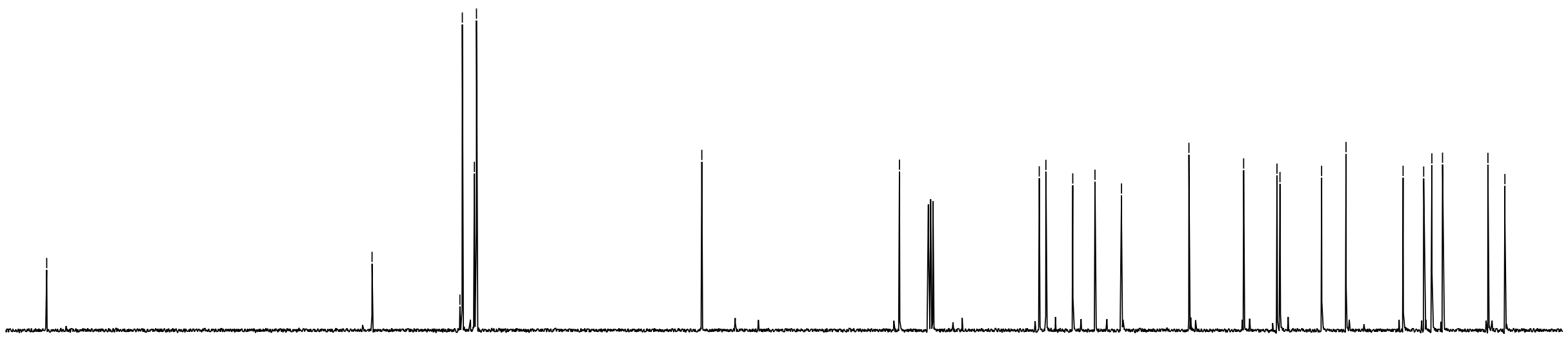

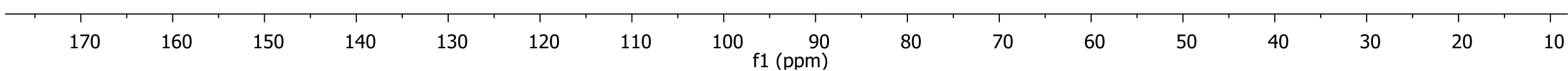


${ }^{1} \mathrm{H}$ NMR $\left(\mathrm{CDCl}_{3}, 500 \mathrm{MHz}\right)$ of compound 3c
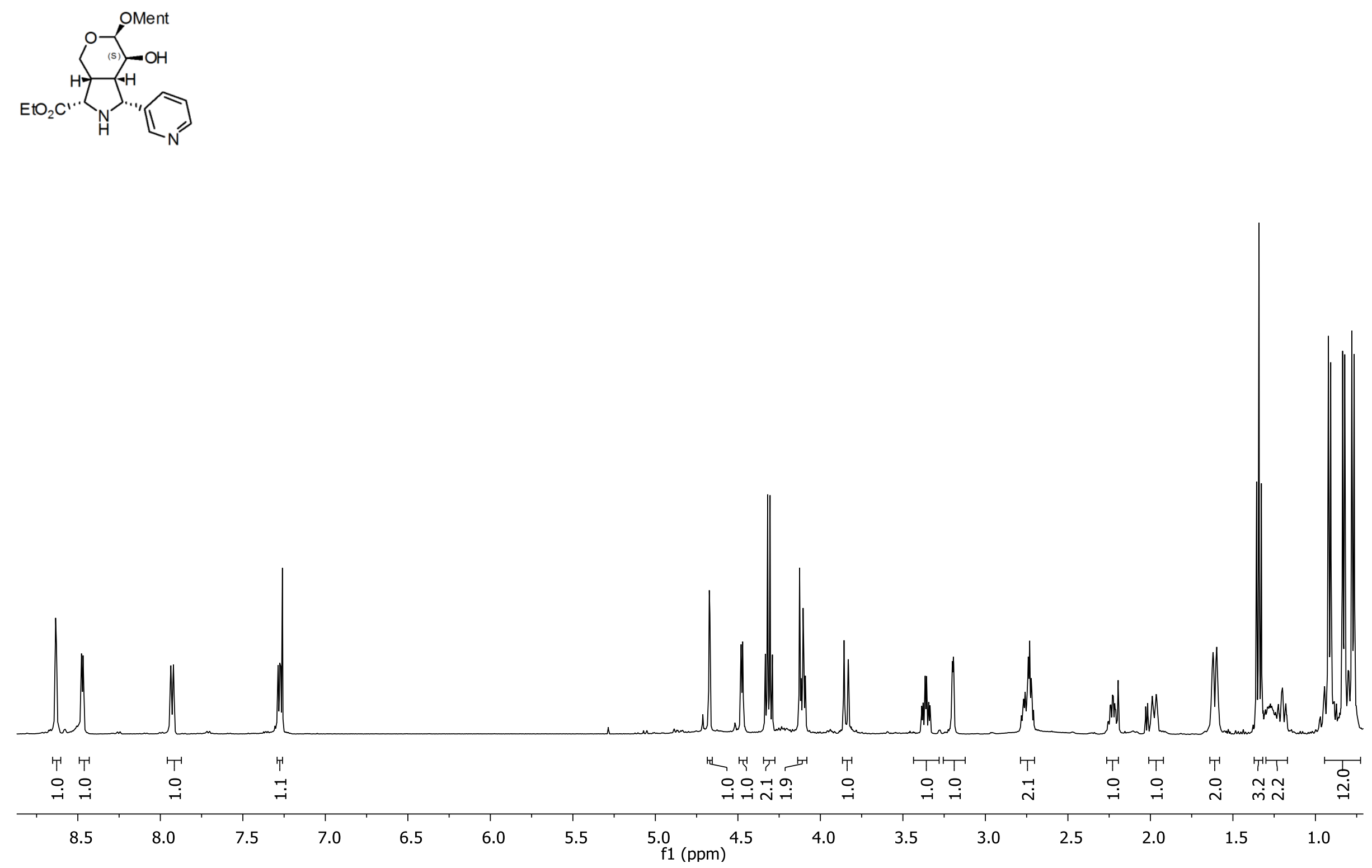
${ }^{13} \mathrm{C} \mathrm{NMR}\left(\mathrm{CDCl}_{3}, 125.7 \mathrm{MHz}\right)$ of compound $\mathbf{3 c}$

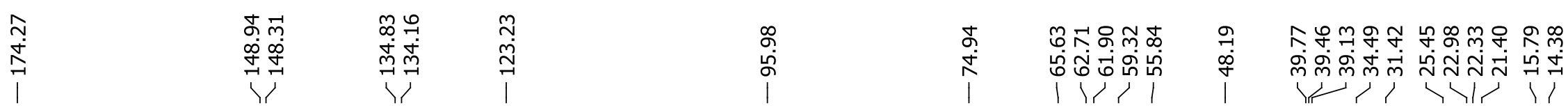

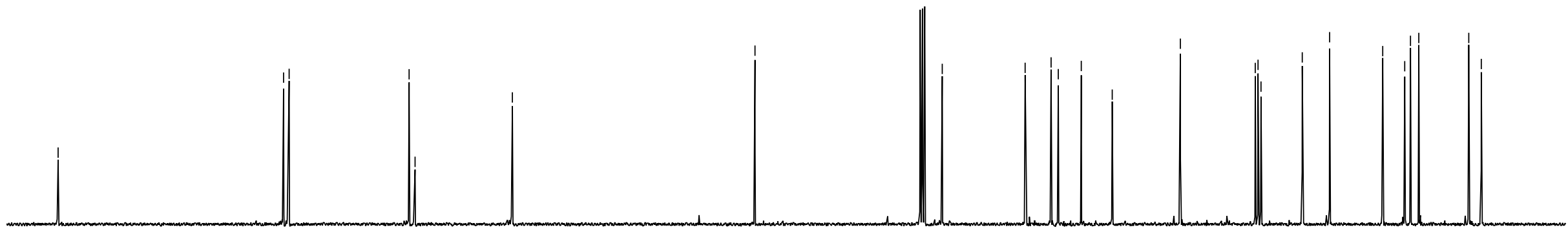

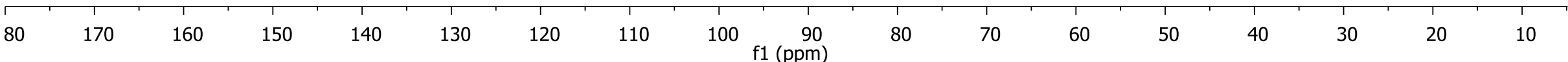


${ }^{1} \mathrm{H}$ NMR $\left(\mathrm{CDCl}_{3}, 500 \mathrm{MHz}\right)$ of compound $\mathbf{3 d}$
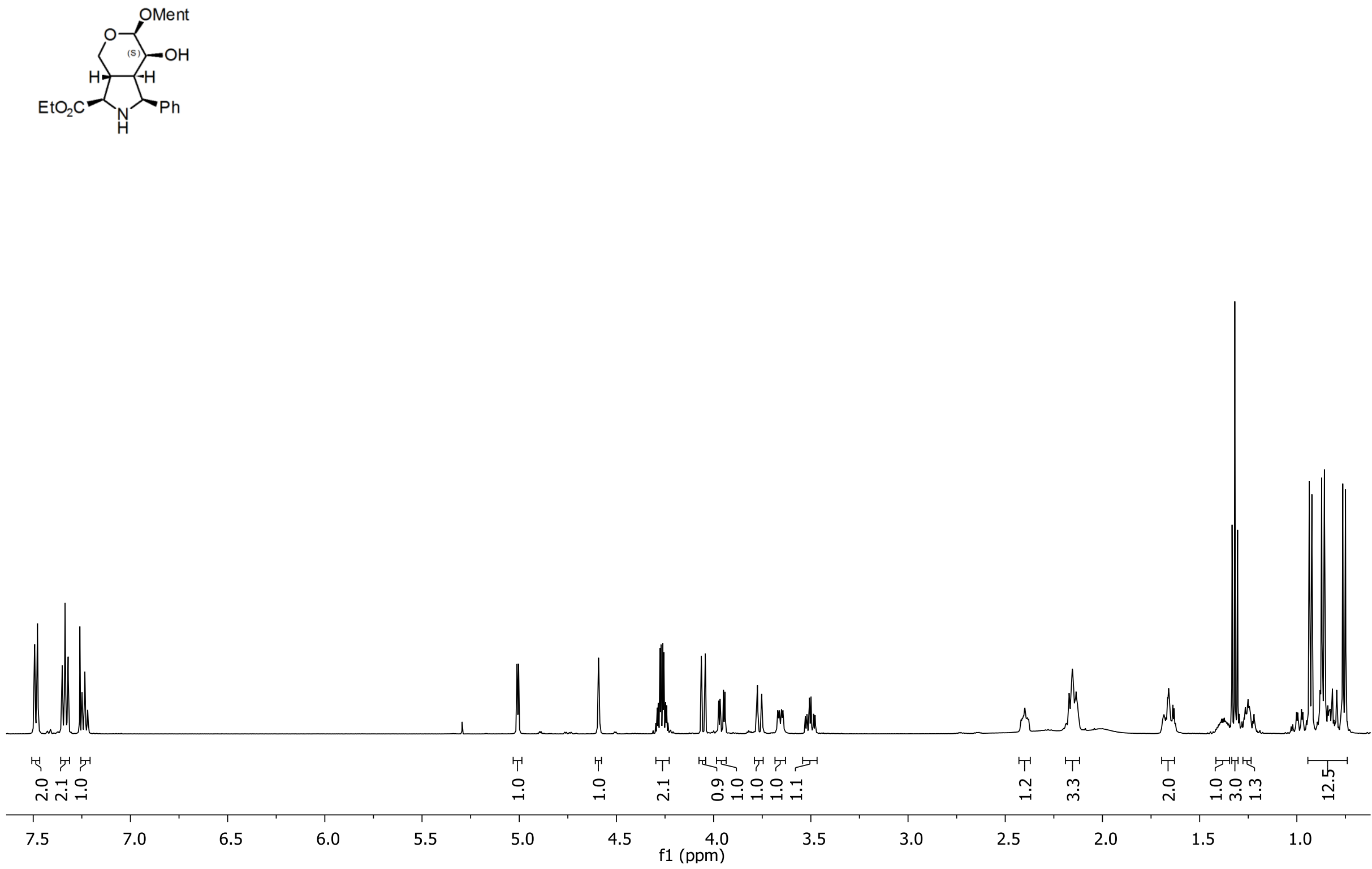
${ }^{13} \mathrm{C} \mathrm{NMR}\left(\mathrm{CDCl}_{3}, 125.7 \mathrm{MHz}\right)$ of compound 3d

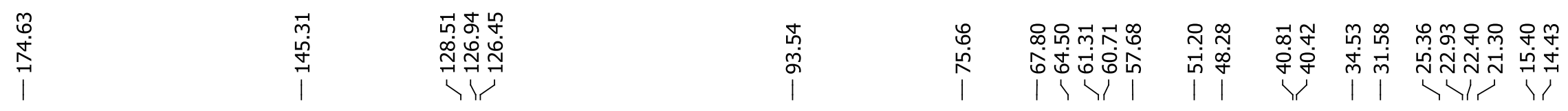

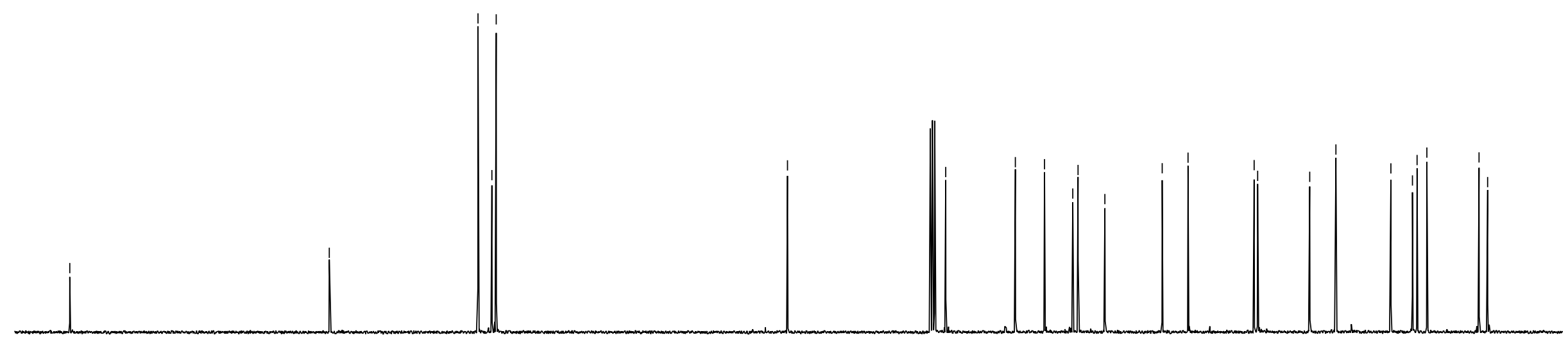

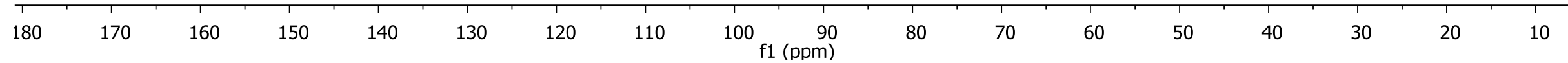


${ }^{1} \mathrm{H}$ NMR $\left(\mathrm{CDCl}_{3}, 500 \mathrm{MHz}\right)$ of compound $\mathbf{3 e}$
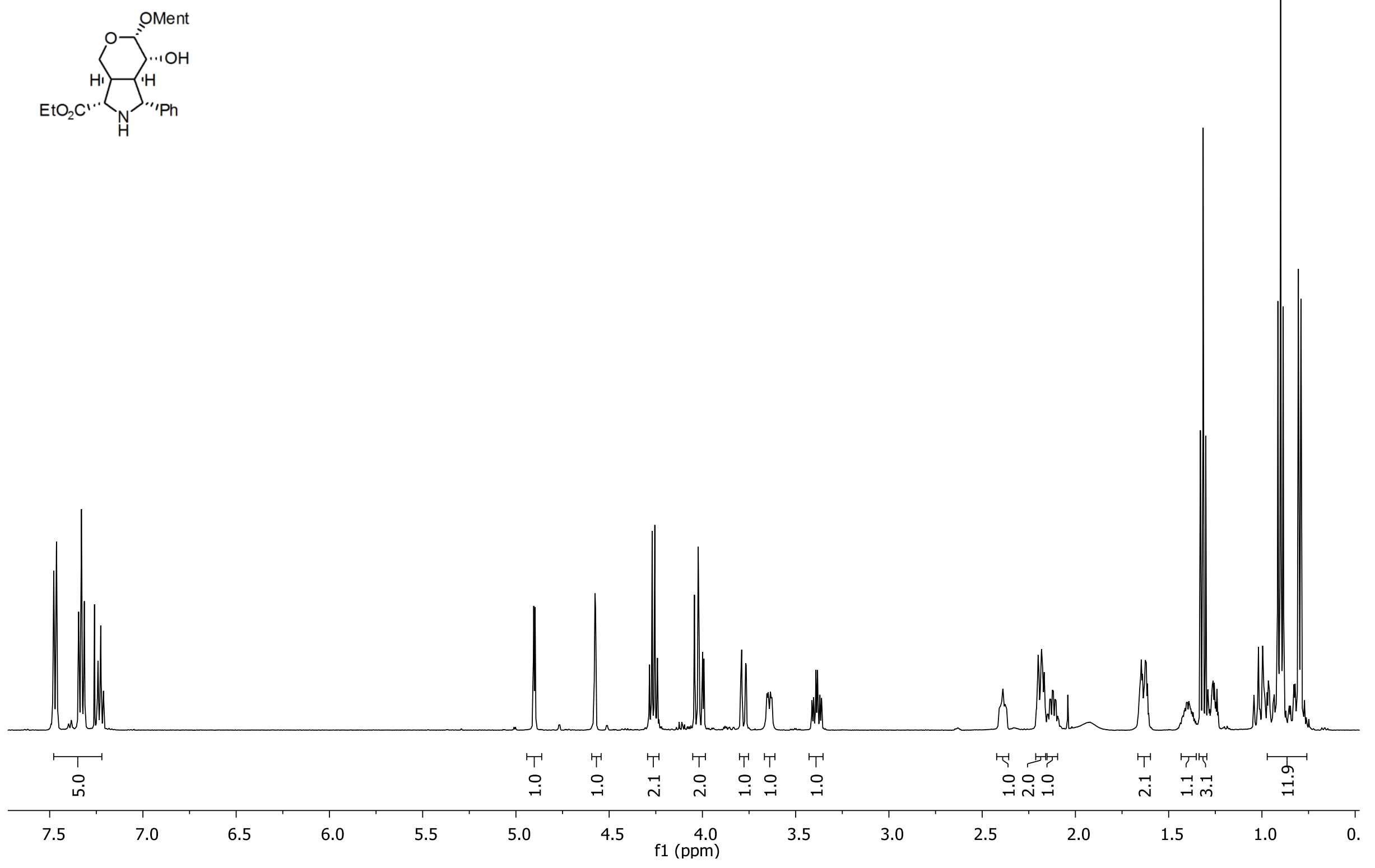
${ }^{13} \mathrm{C}$ NMR $(\mathrm{CDCl}, 125.7 \mathrm{MHz})$ of compound $3 \mathrm{e}$

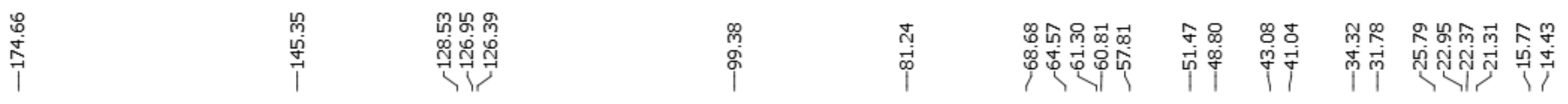

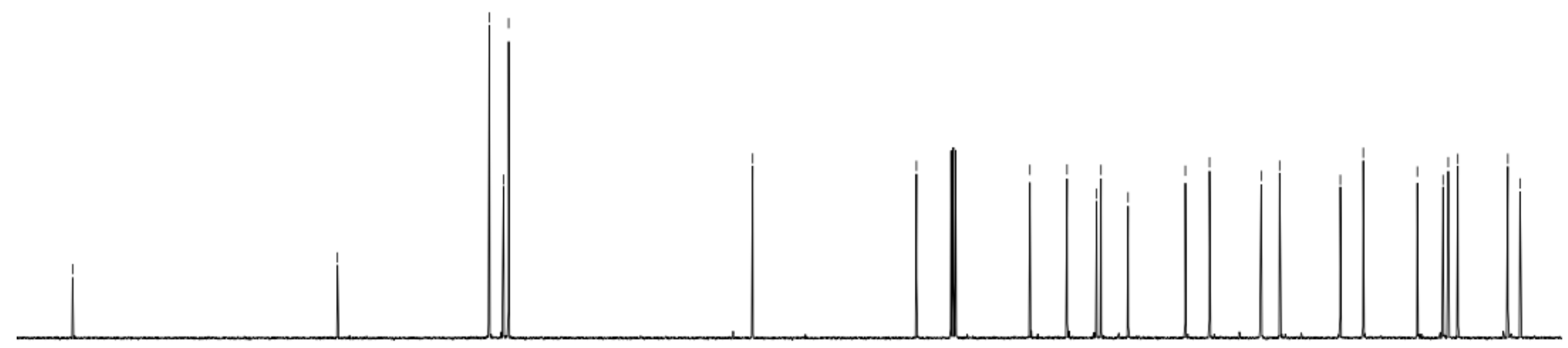


${ }^{1} \mathrm{H} \mathrm{NMR}\left(\mathrm{CDCl}_{3}, 500 \mathrm{MHz}\right)$ of compound $\mathbf{3 f}$
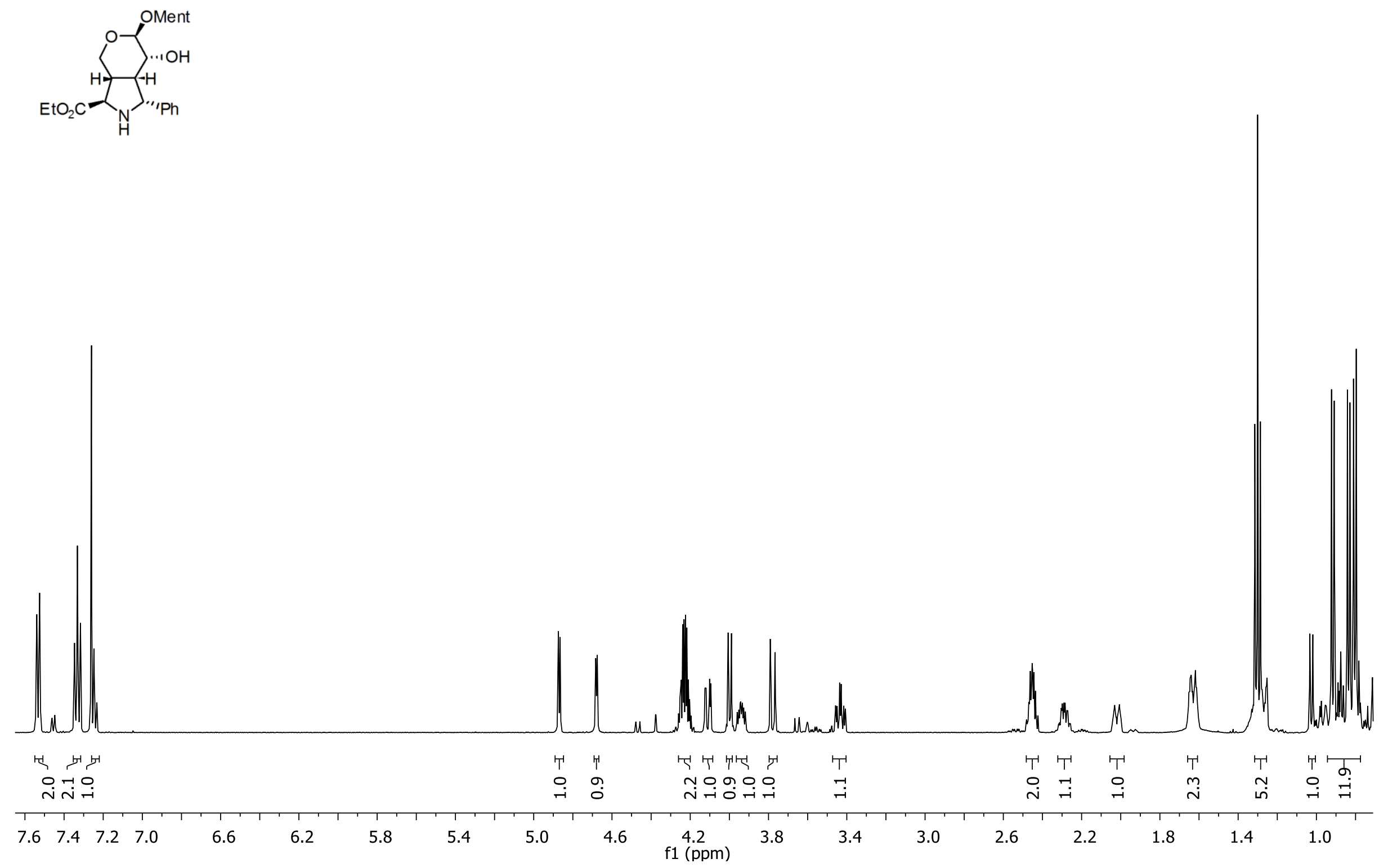
${ }^{13} \mathrm{C}$ NMR $\left(\mathrm{CDCl}_{3}, 125.7 \mathrm{MHz}\right)$ of compound $\mathbf{3 f}$

\begin{tabular}{|c|c|c|c|c|c|c|c|c|}
\hline $\begin{array}{l}5 \\
\vdots \\
\stackrel{0}{0} \\
1\end{array}$ & & 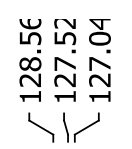 & 负 & 量 & 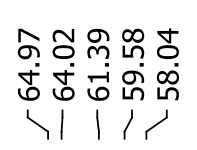 & 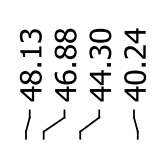 & 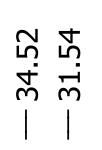 & 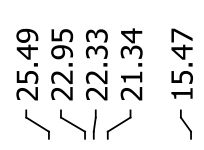 \\
\hline
\end{tabular}

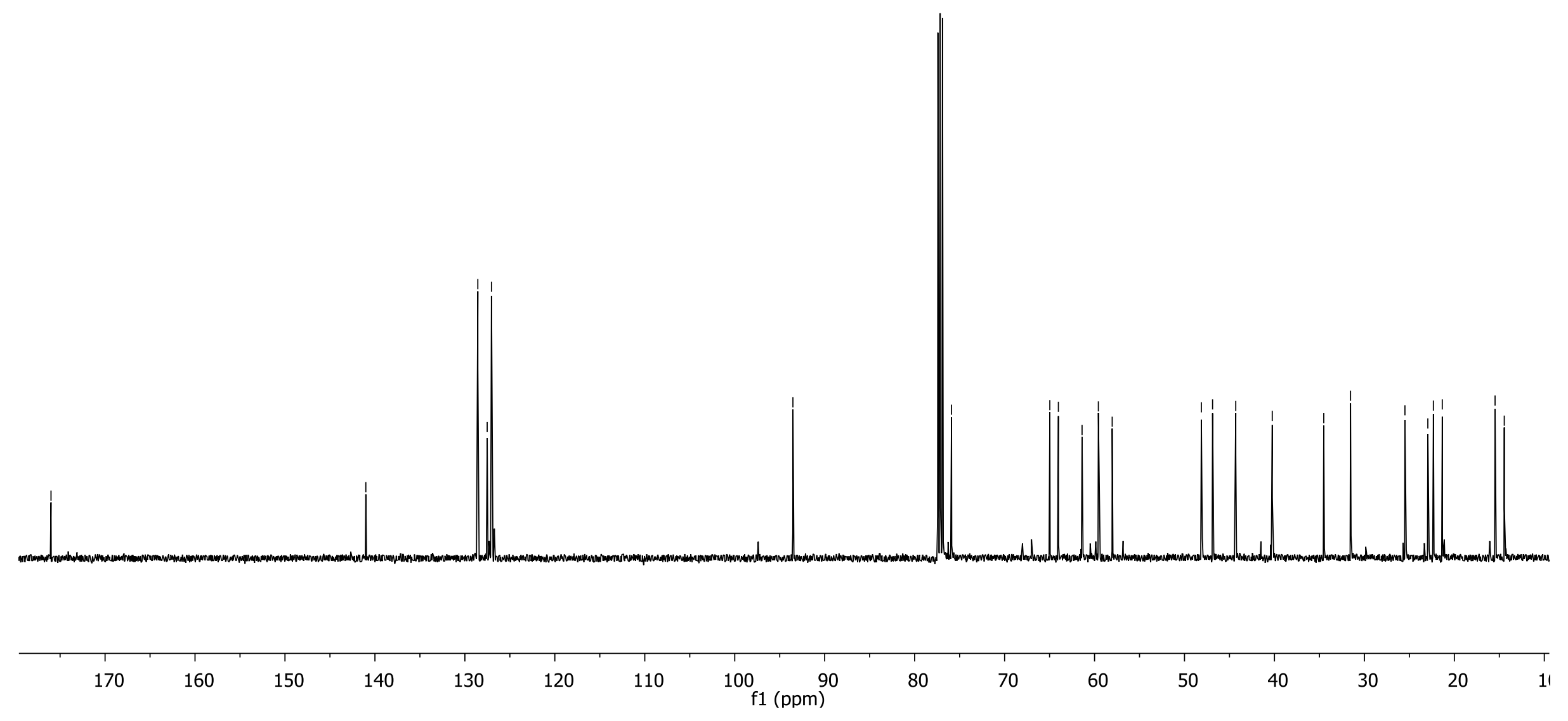


${ }^{1} \mathrm{H} \mathrm{NMR}\left(\mathrm{CDCl}_{3}, 500 \mathrm{MHz}\right)$ of compound $\mathbf{4 f}$
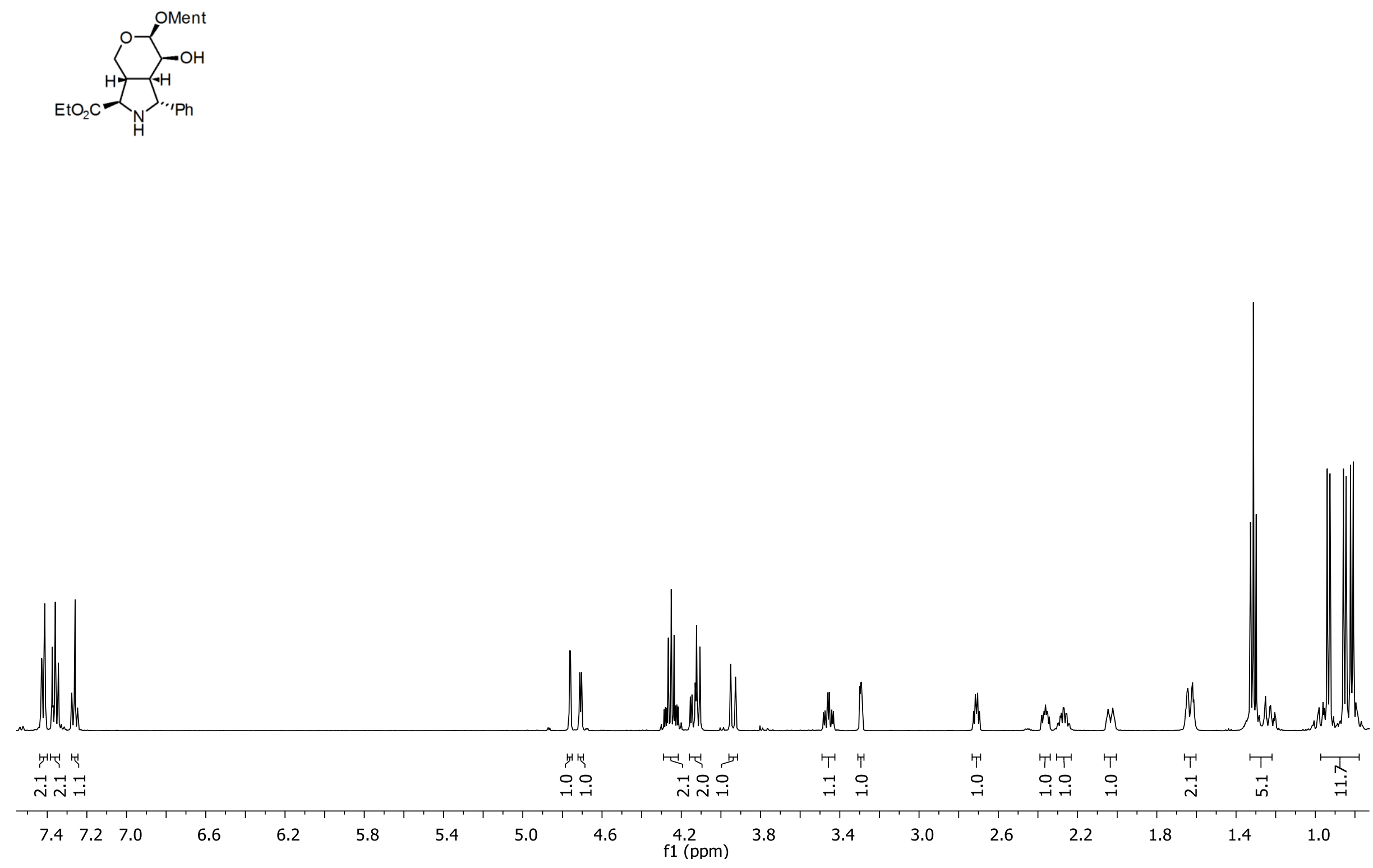
${ }^{13} \mathrm{C} \mathrm{NMR}\left(\mathrm{CDCl}_{3}, 125.7 \mathrm{MHz}\right)$ of compound $\mathbf{4 f}$

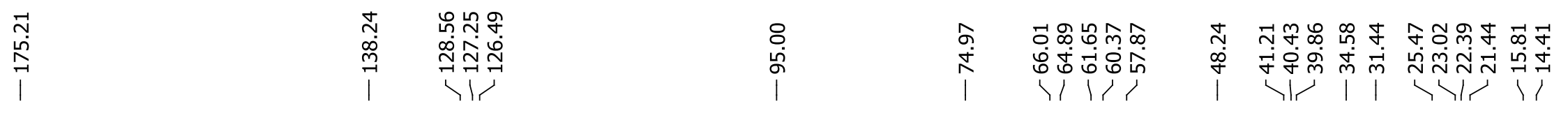

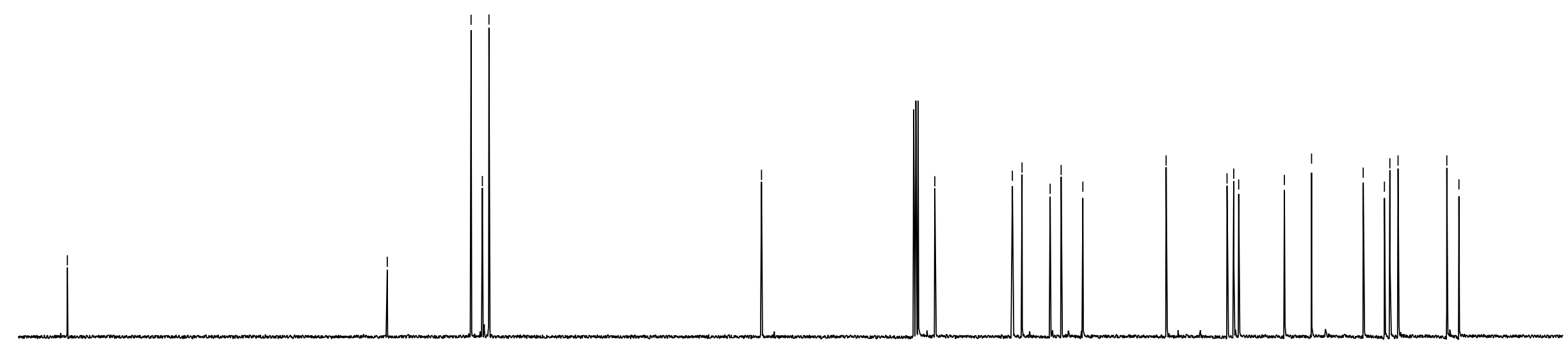

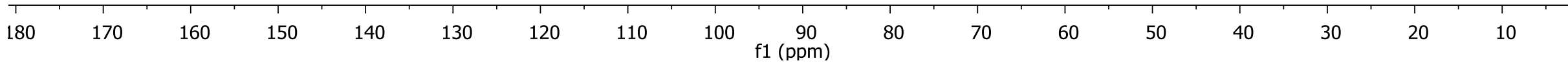


${ }^{1} \mathrm{H} \mathrm{NMR}\left(\mathrm{CDCl}_{3}, 500 \mathrm{MHz}\right)$ of compound $\mathbf{5 a}$
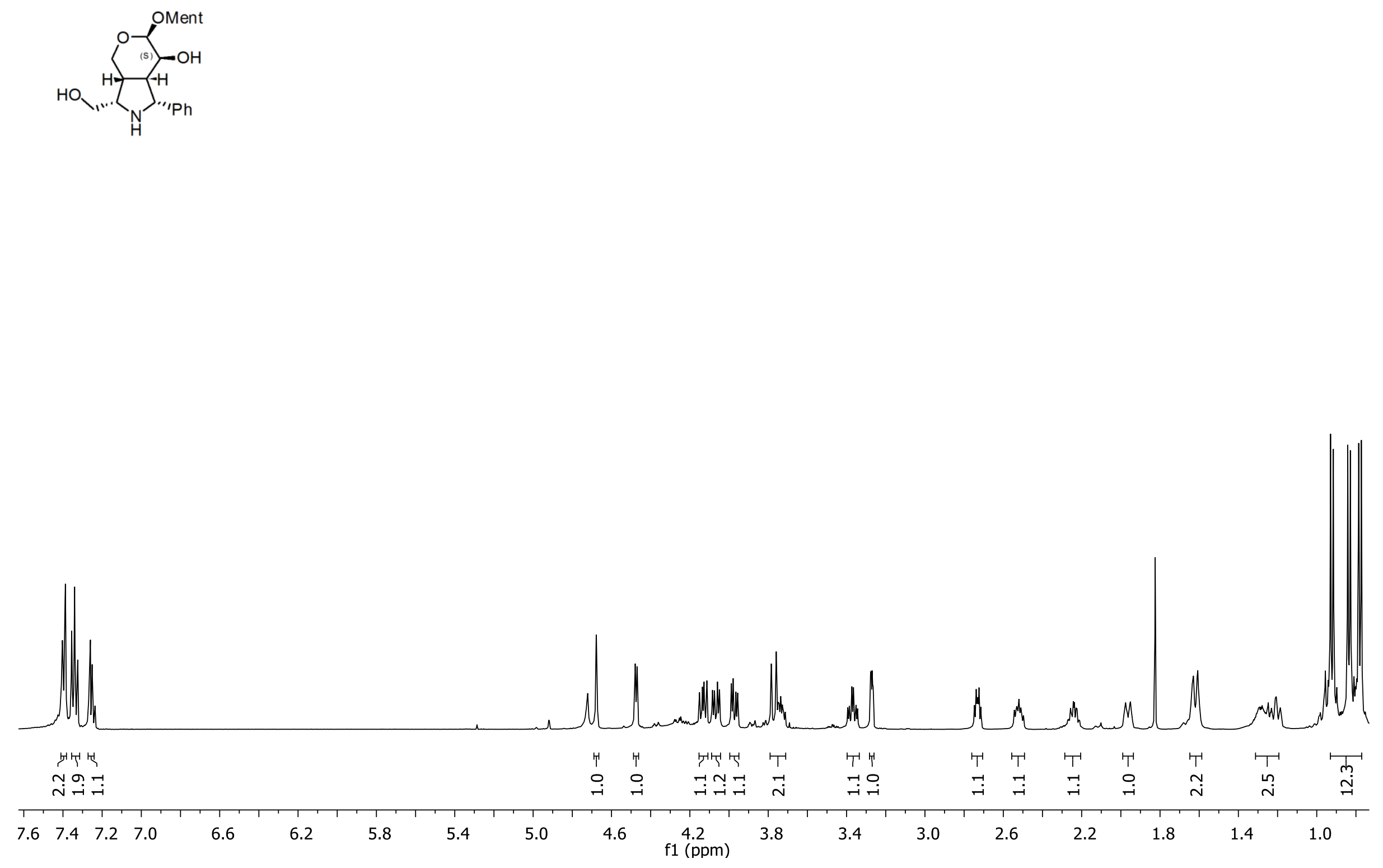
${ }^{13} \mathrm{C}$ NMR $\left(\mathrm{CDCl}_{3}, 125.7 \mathrm{MHz}\right)$ of compound $\mathbf{5 a}$

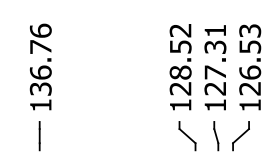

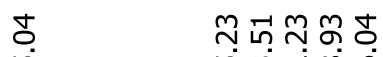

年

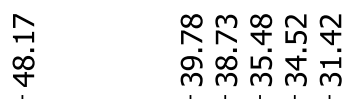

뭉ํํㅇ

ผั่ํำ

।रा रूथा ।

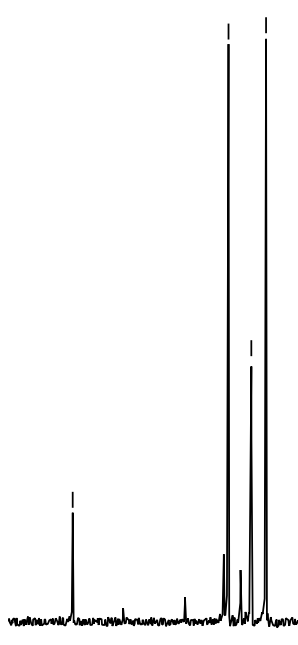

$40 \quad 135$

130

125

120

115

$110 \quad 105 \quad 100$

9590

85

$\begin{array}{lc}80 & 75 \\ \mathrm{f} 1(\mathrm{ppm})\end{array}$

$65 \quad 60$

55

$50 \quad 45$

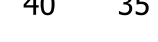

30

$\begin{array}{lll}25 & 20 \quad 15\end{array}$ 
${ }^{1} \mathrm{H} \mathrm{NMR}\left(\mathrm{CDCl}_{3}, 500 \mathrm{MHz}\right)$ of compound $\mathbf{5 c}$
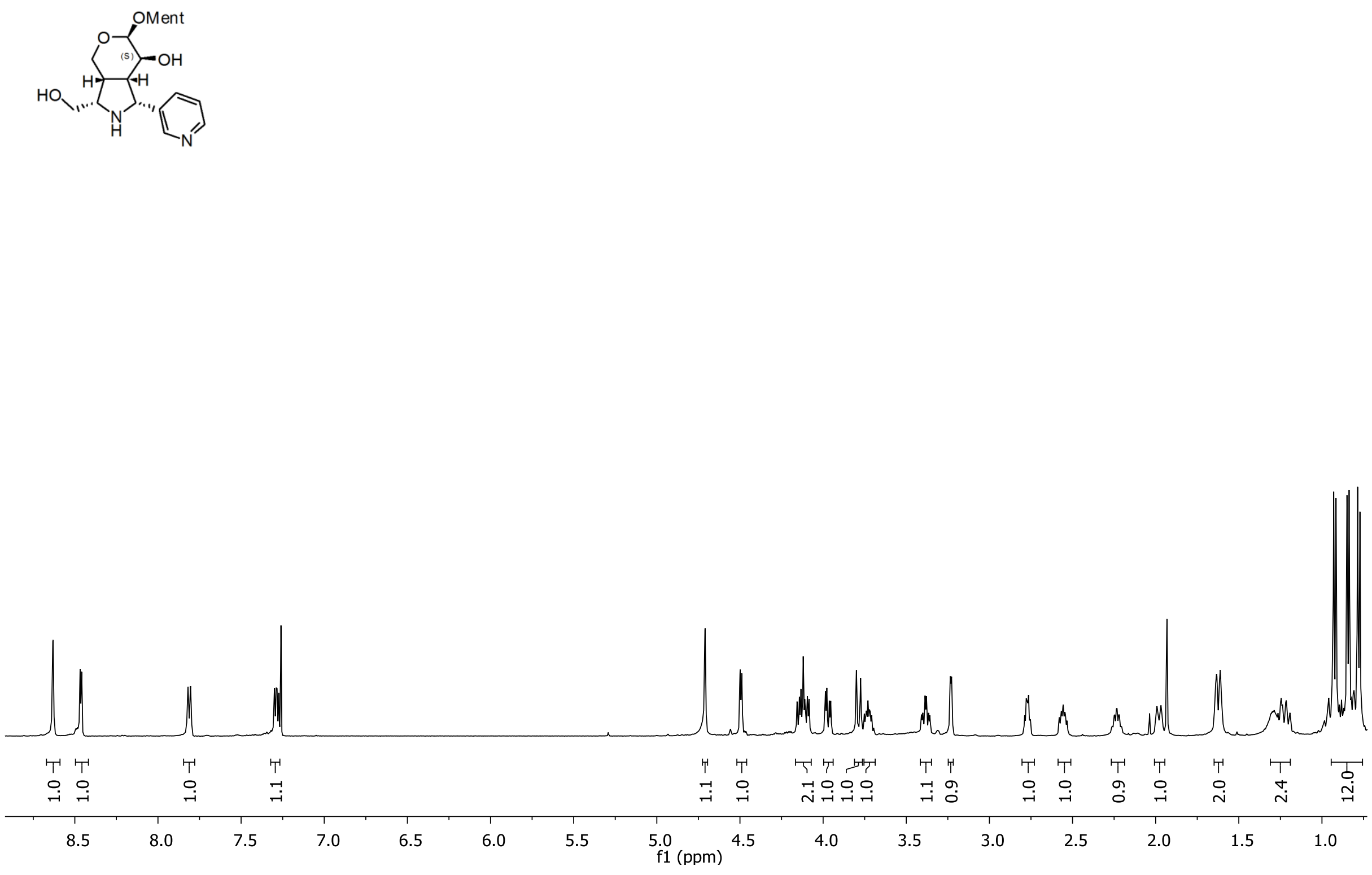
${ }^{13} \mathrm{C} \mathrm{NMR}\left(\mathrm{CDCl}_{3}, 125.7 \mathrm{MHz}\right)$ of compound $\mathbf{5 c}$

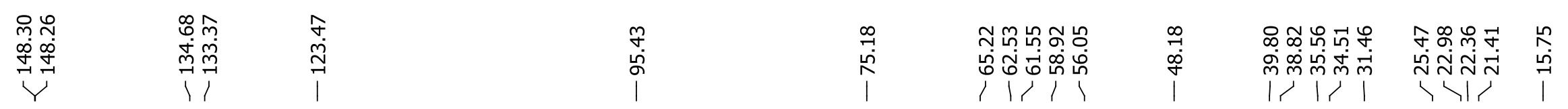

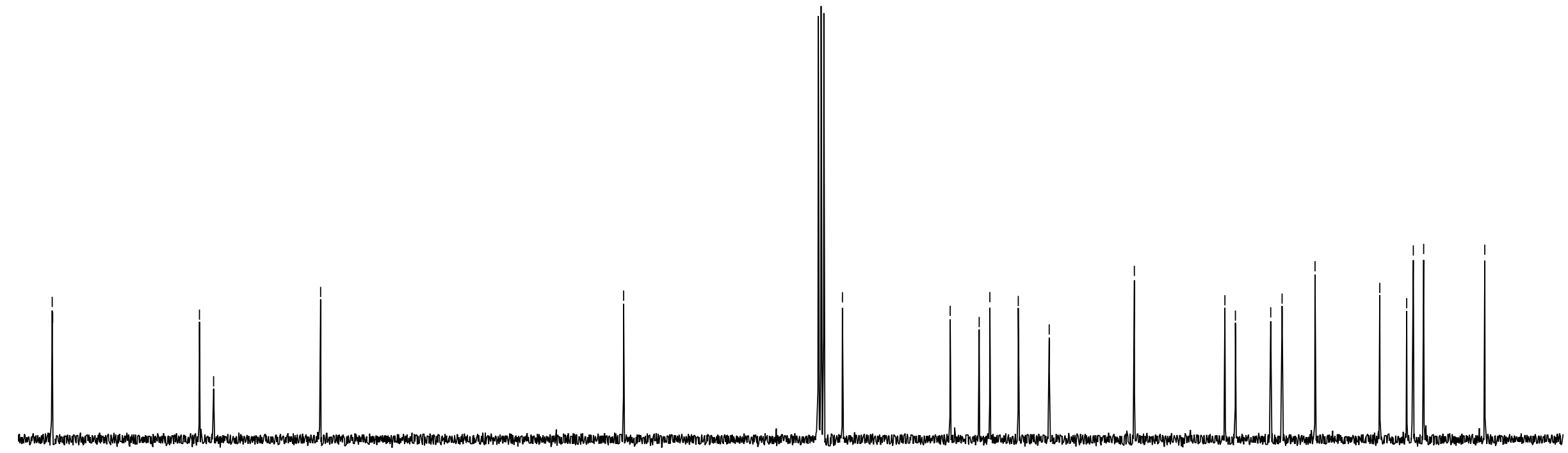

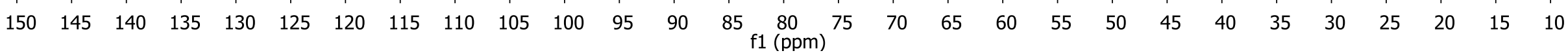


${ }^{1} \mathrm{H}$ NMR (pyridine- $d_{5}, 500 \mathrm{MHz}$ ) of compound 6a

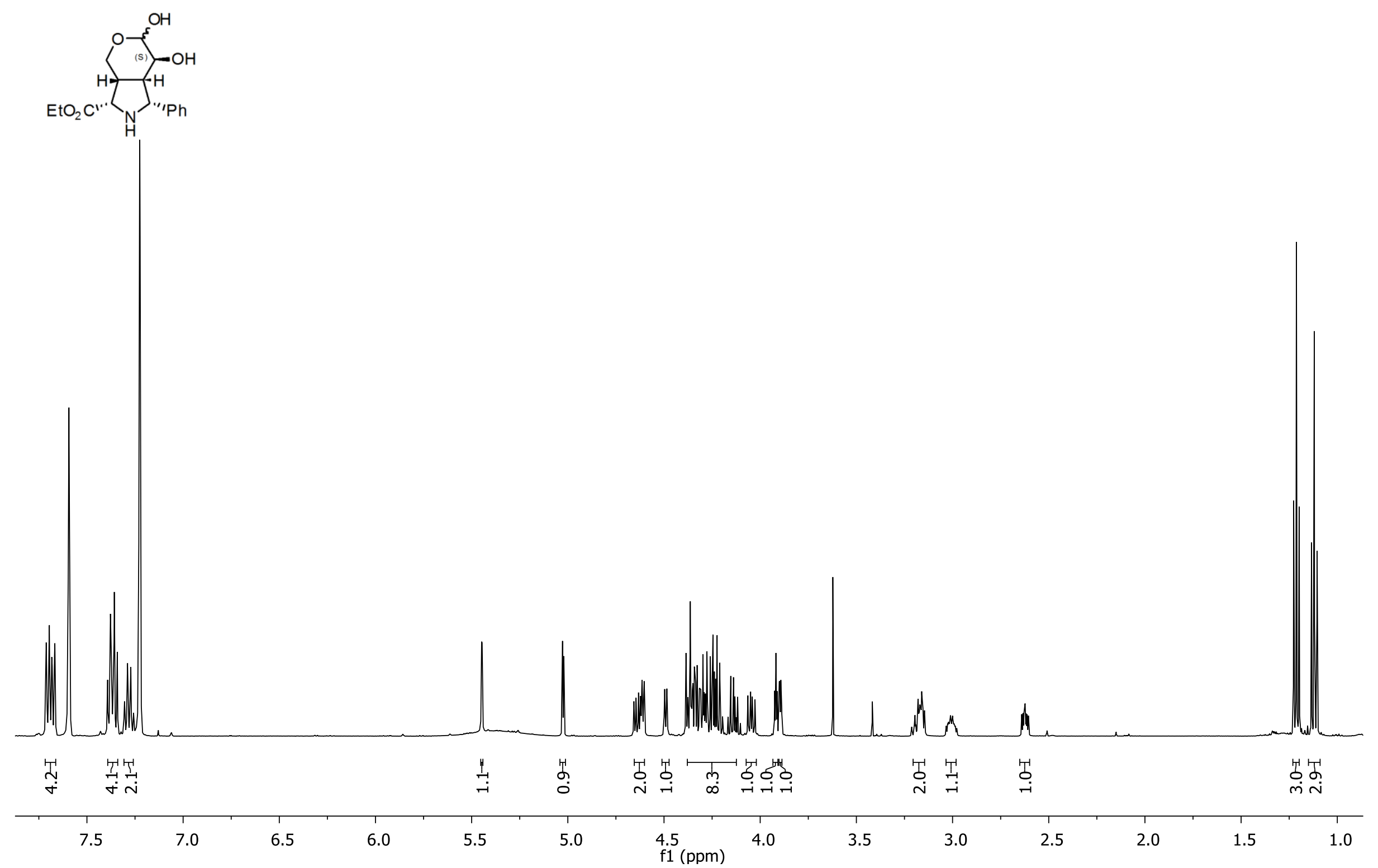


${ }^{13} \mathrm{C}$ NMR (pyridine- $d_{5}, 125.7 \mathrm{MHz}$ ) of compound 6a

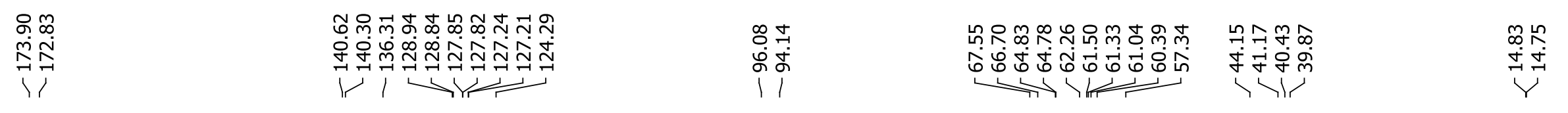

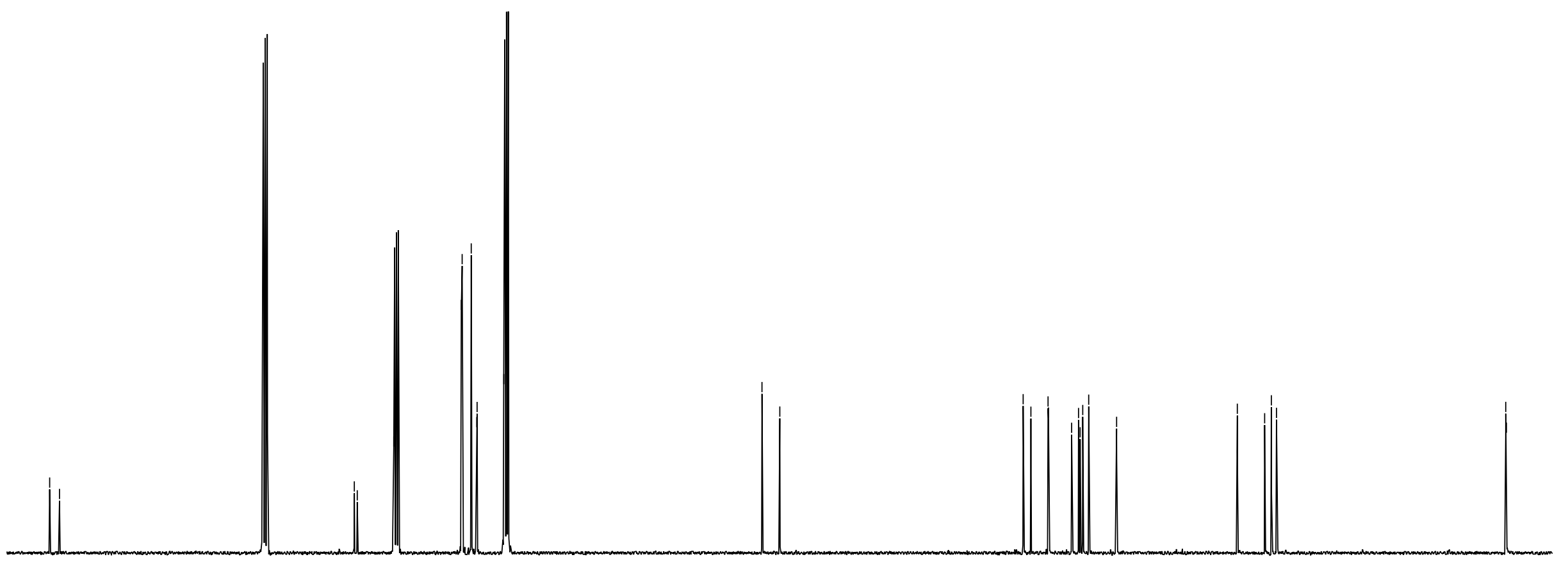




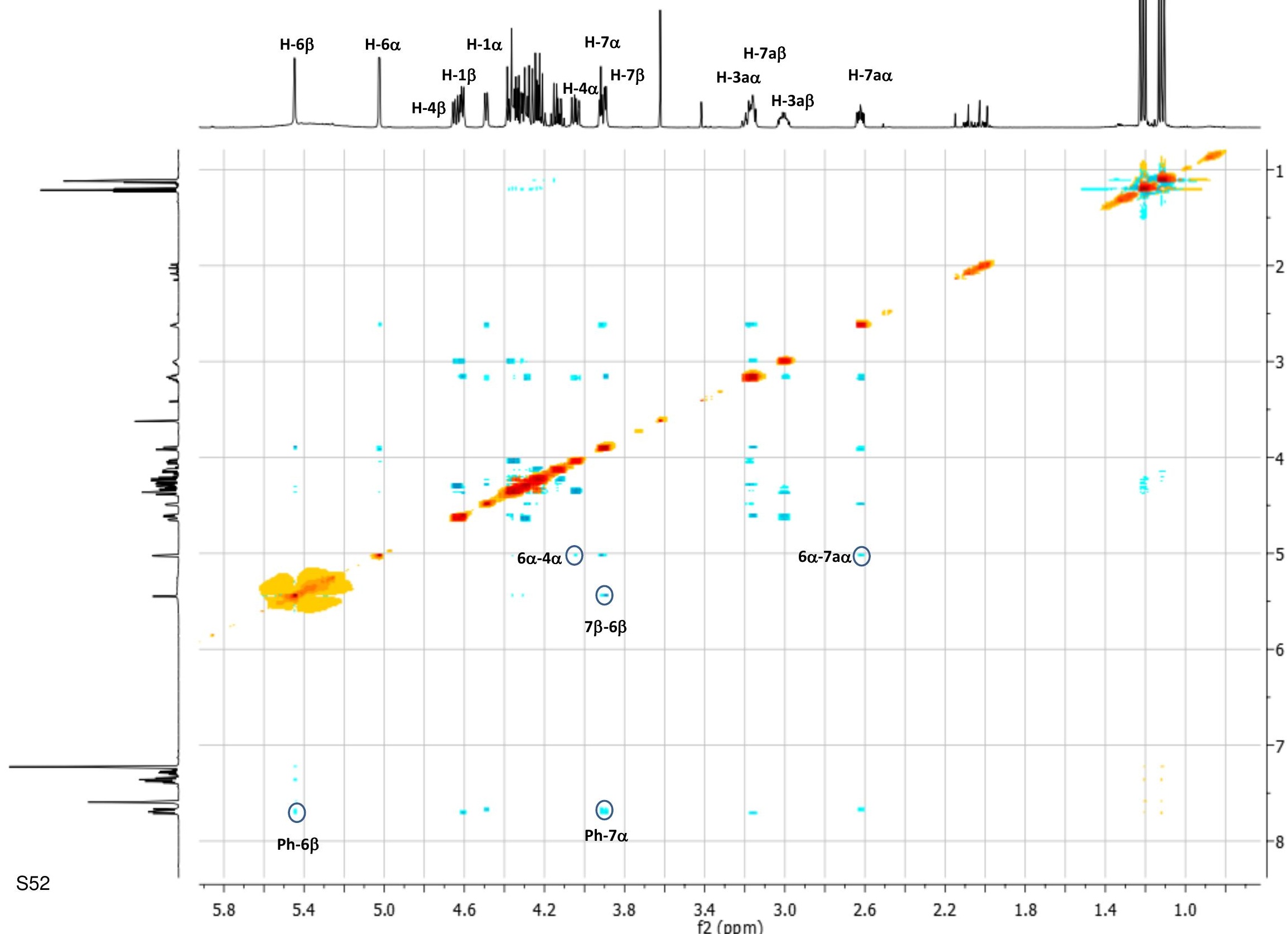


${ }^{1} \mathrm{H}$ NMR $\left(\mathrm{CDCl}_{3}, 500 \mathrm{MHz}\right)$ of compound 7a
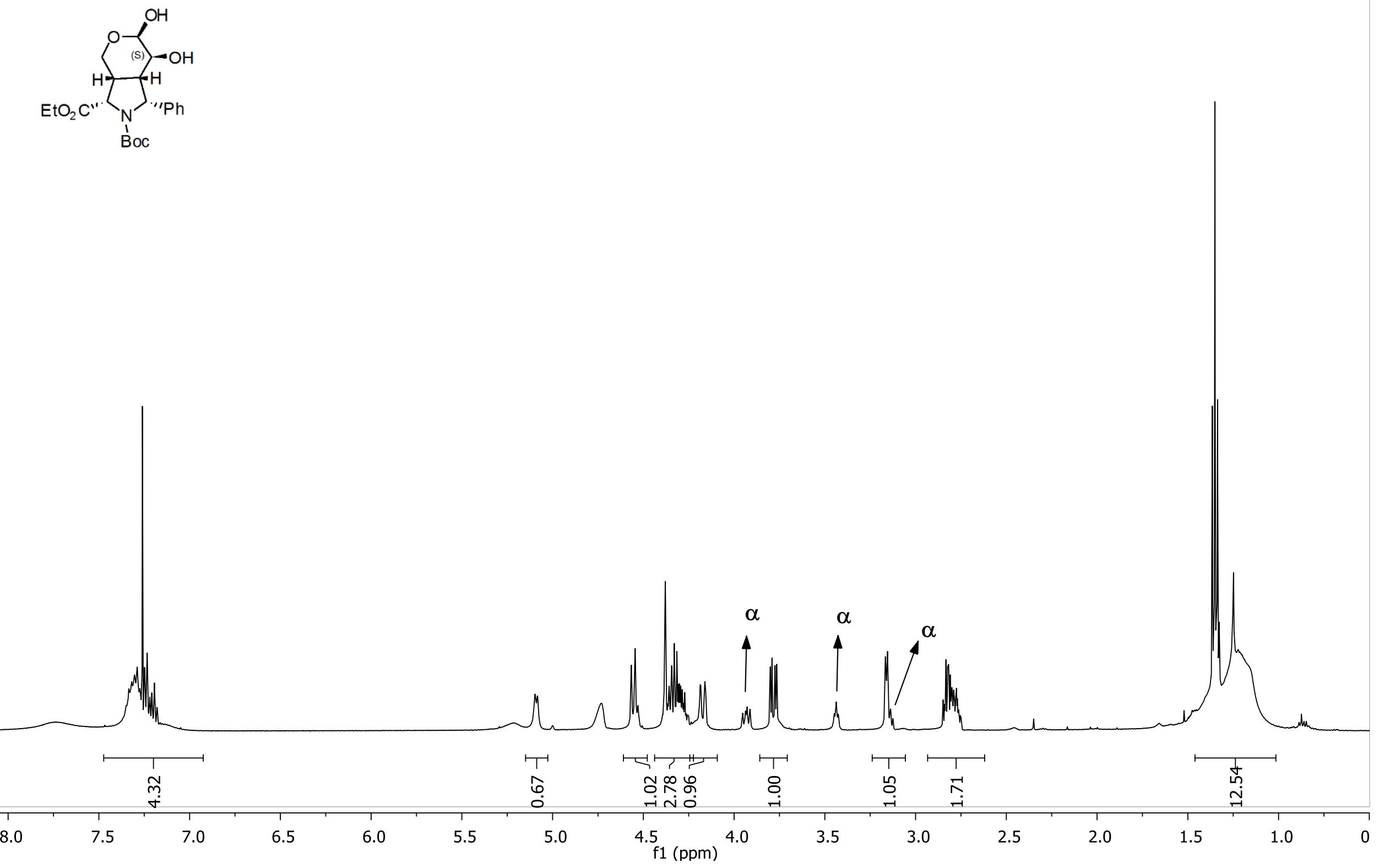
${ }^{13} \mathrm{C}$ NMR $\left(\mathrm{CDCl}_{3}, 125.7 \mathrm{MHz}\right)$ of compound 7a
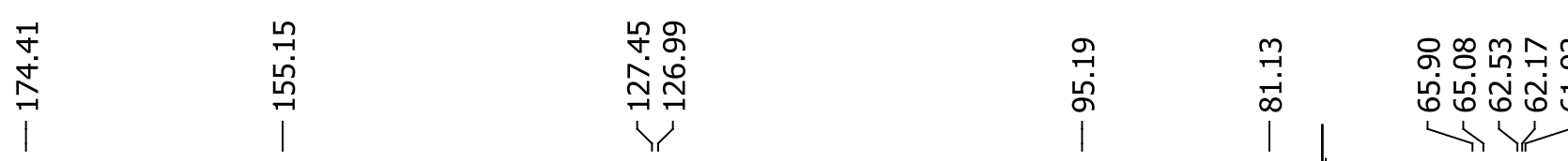

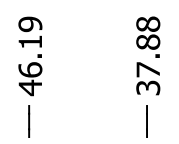

§ั

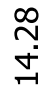

0

|

\begin{tabular}{|c|c|c|c|c|c|c|c|c|c|c|c|c|c|c|c|c|}
\hline 180 & 170 & 160 & 150 & 140 & 130 & 120 & 110 & $\begin{array}{c}100 \\
\mathrm{f} 1(\mathrm{ppm})\end{array}$ & 80 & 70 & 60 & 50 & 40 & 30 & 20 & 10 \\
\hline & & & & & & & & S54 & & & & & & & & \\
\hline
\end{tabular}


${ }^{1} \mathrm{H} \mathrm{NMR}\left(\mathrm{CDCl}_{3}, 500 \mathrm{MHz}\right)$ of compound 10a

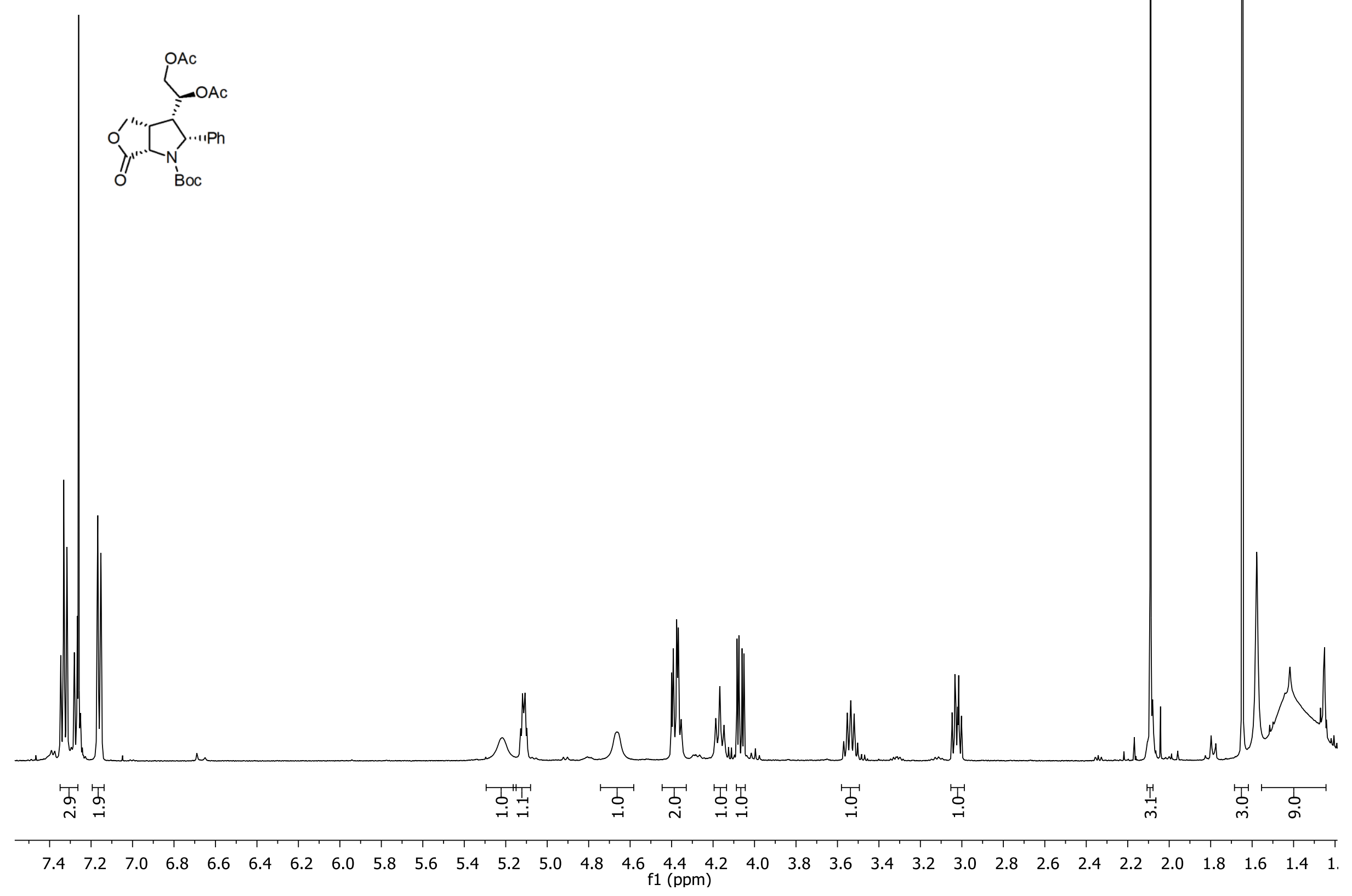


${ }^{13} \mathrm{C} \mathrm{NMR}\left(\mathrm{CDCl}_{3}, 125.7 \mathrm{MHz}\right)$ of compound 10a

\begin{tabular}{|c|c|}
\hline 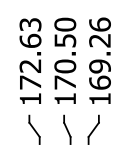 & \\
\hline
\end{tabular}

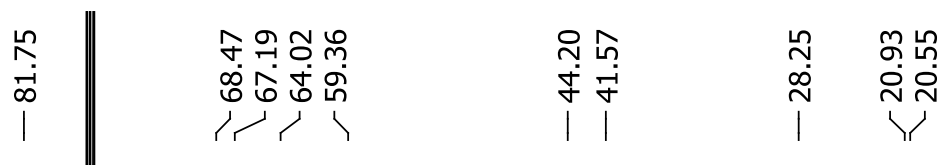

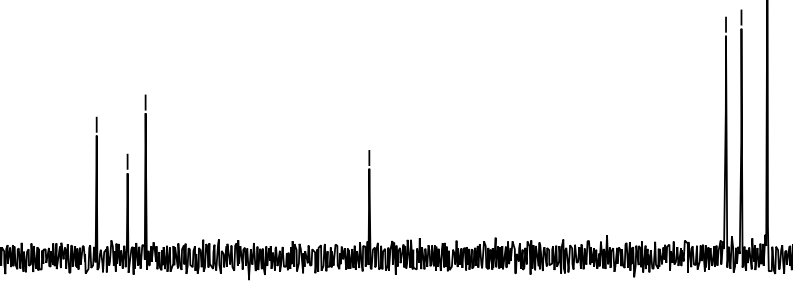

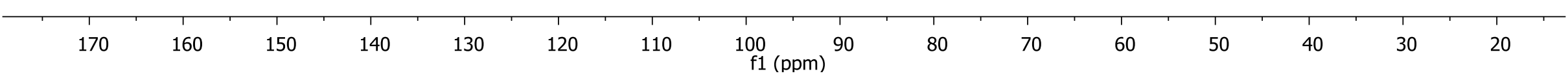




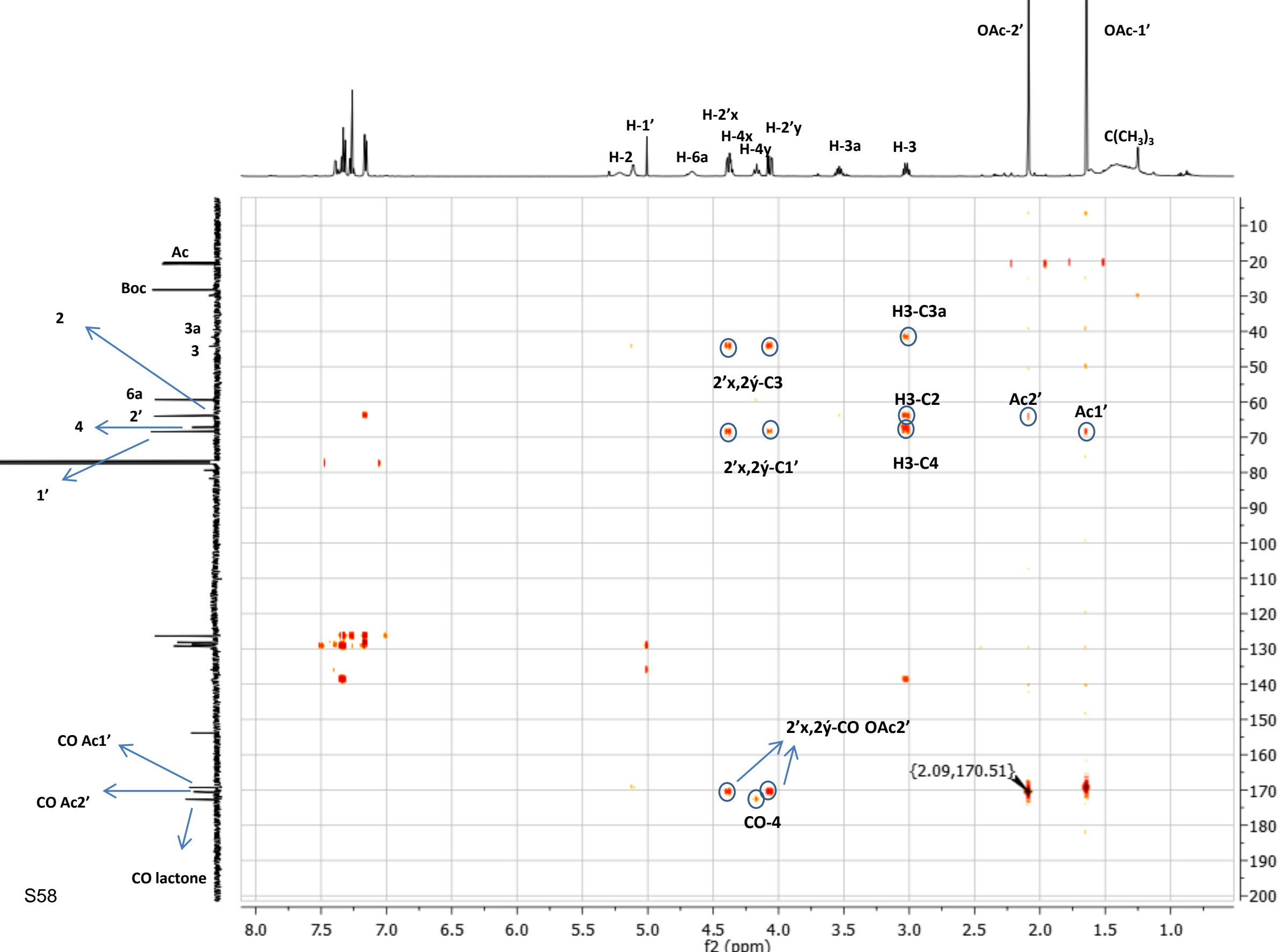


${ }^{1} \mathrm{H}$ NMR $\left(\mathrm{CDCl}_{3}, 500 \mathrm{MHz}\right)$ of compound 11 a
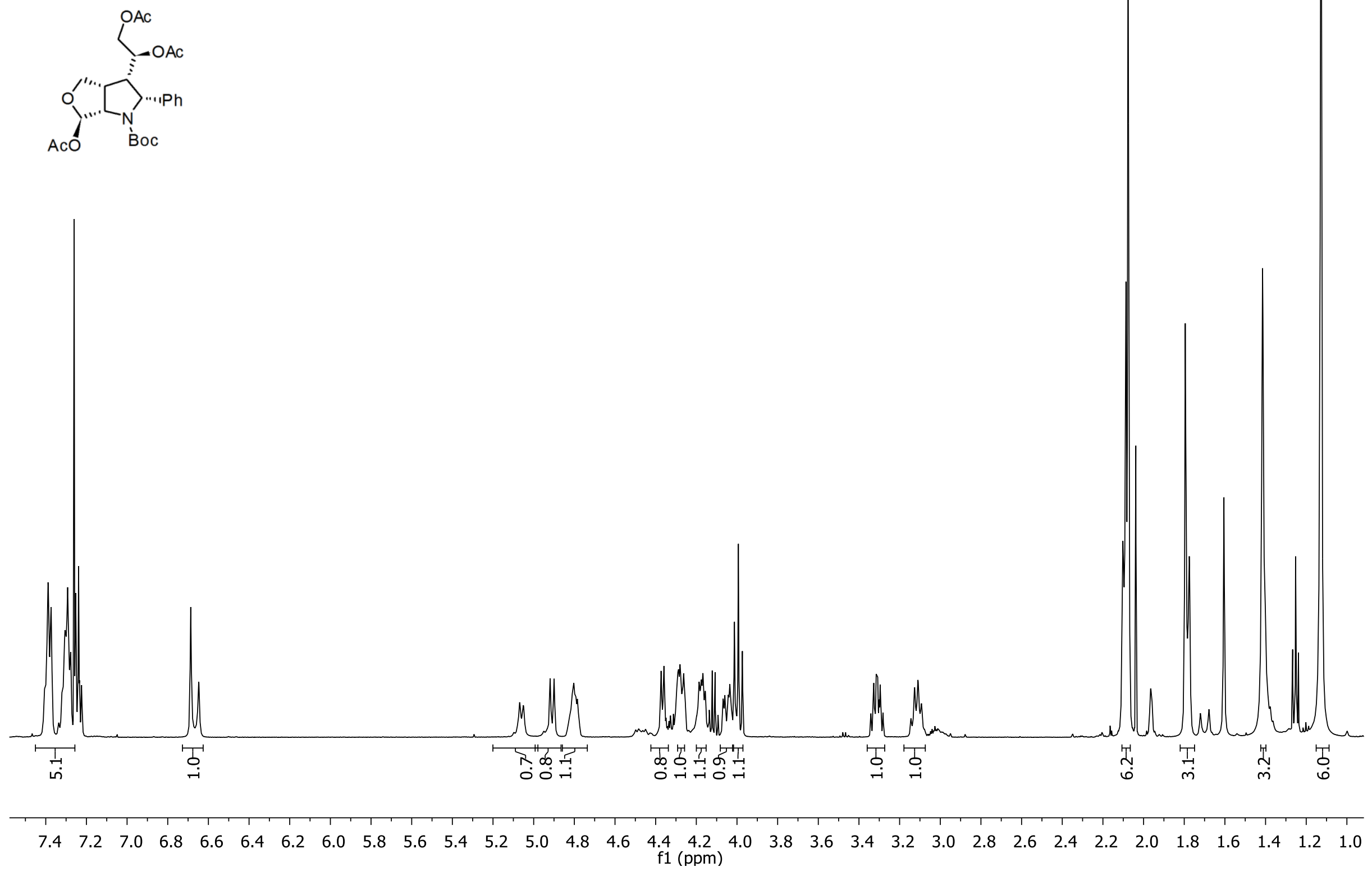

S59 


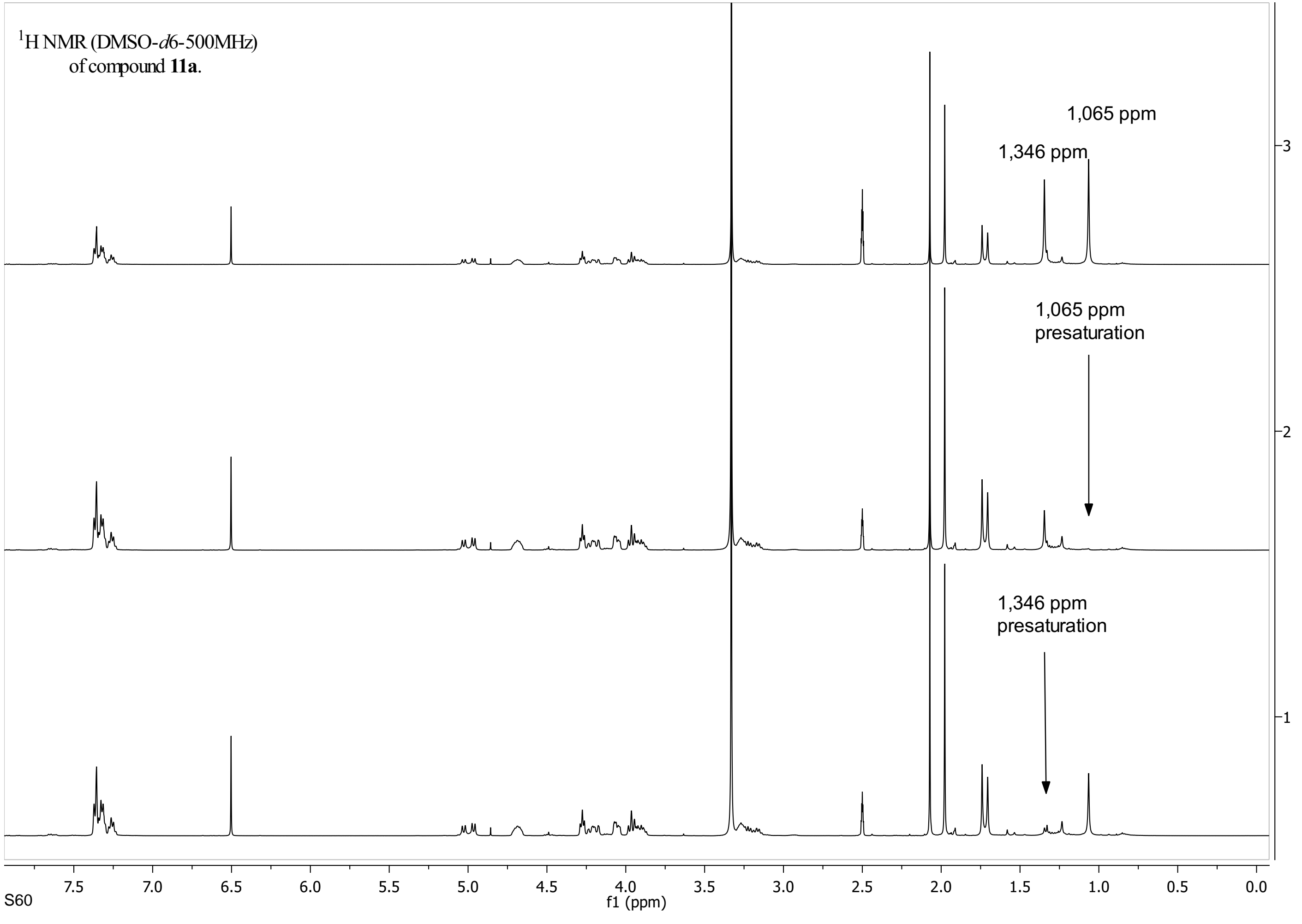


${ }^{13} \mathrm{C} \mathrm{NMR}\left(\mathrm{CDCl}_{3}, 125.7 \mathrm{MHz}\right)$ of compound 11a

\begin{tabular}{|c|c|c|}
\hline 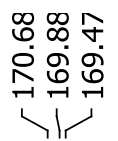 & 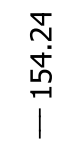 & 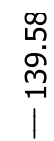 \\
\hline
\end{tabular}

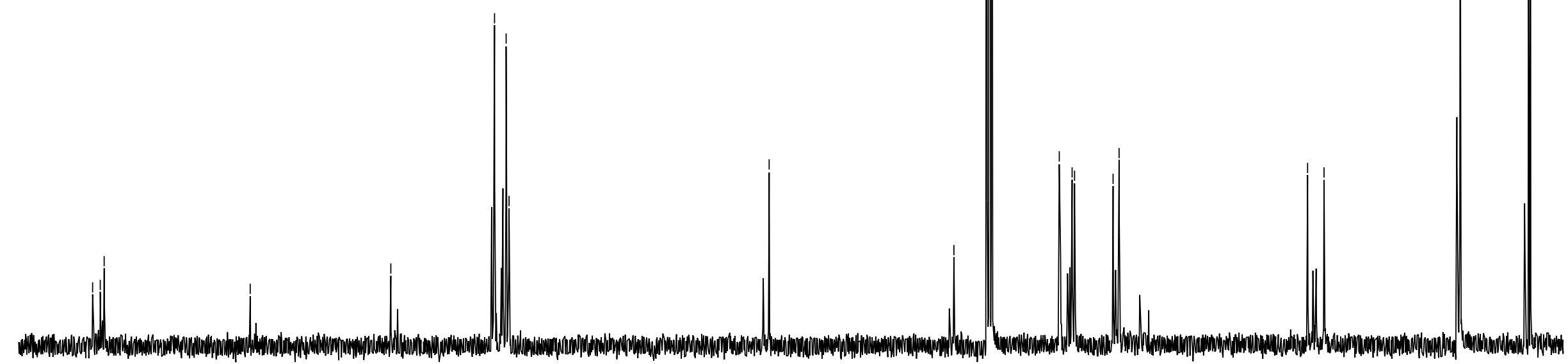




\section{H-6major}

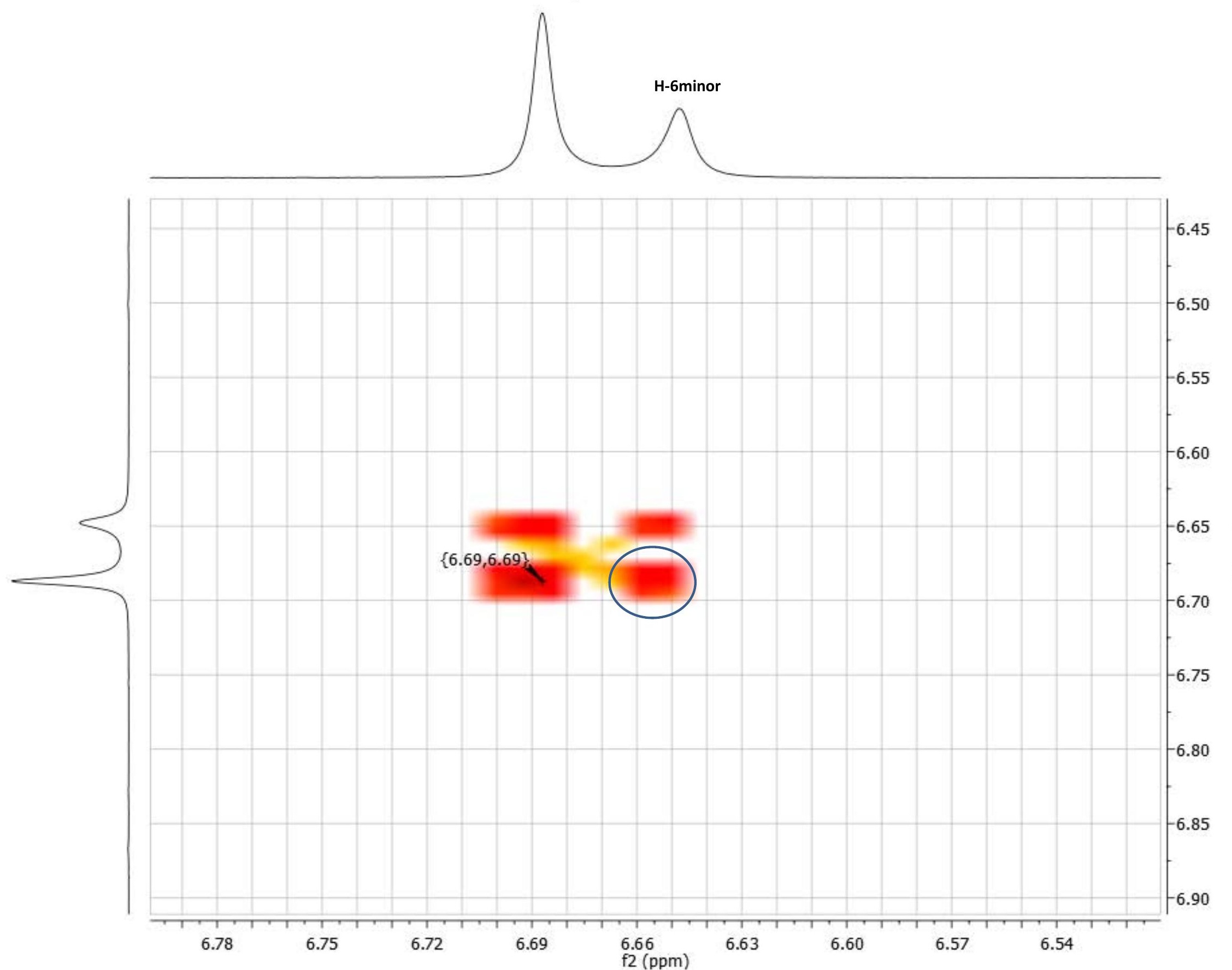




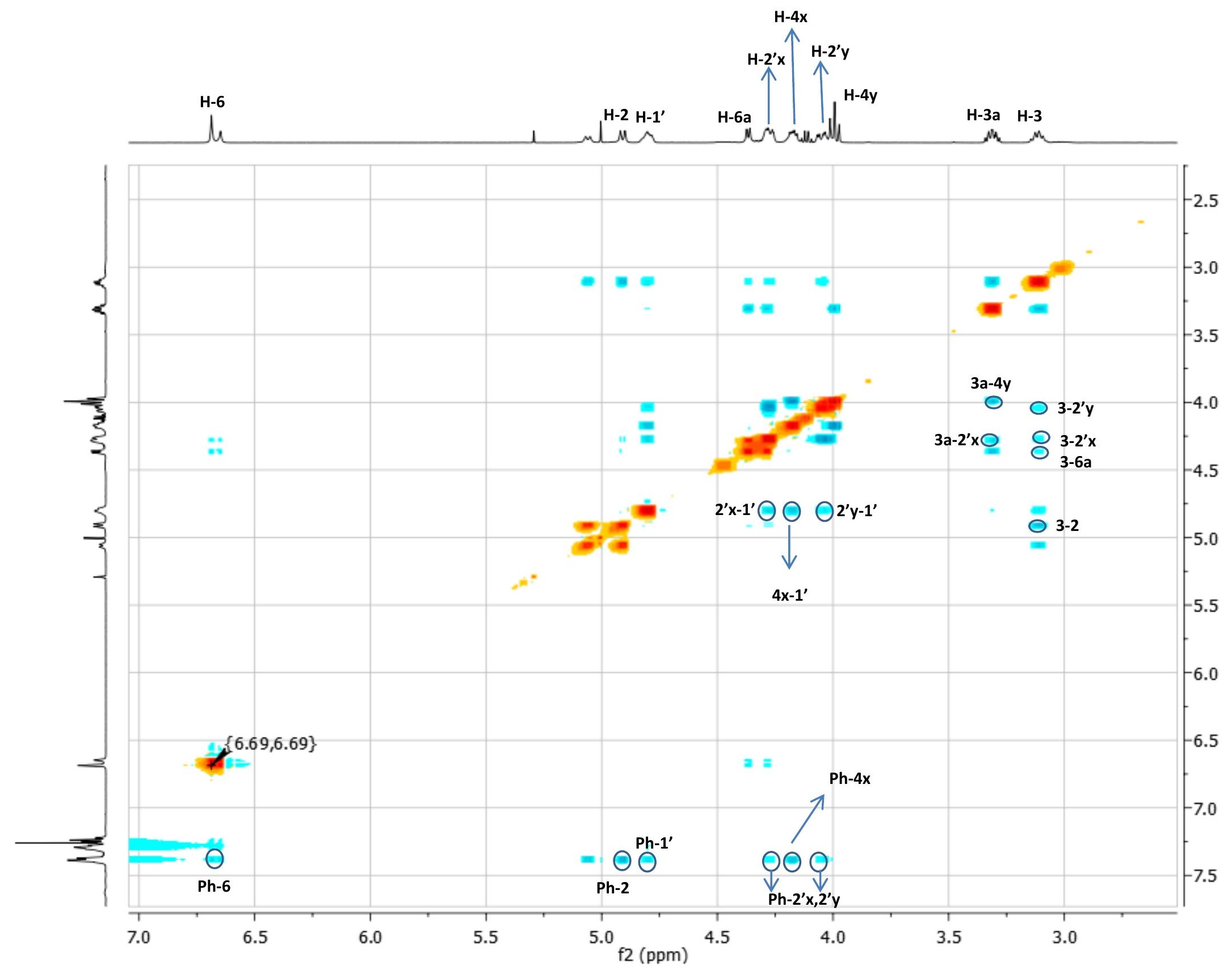




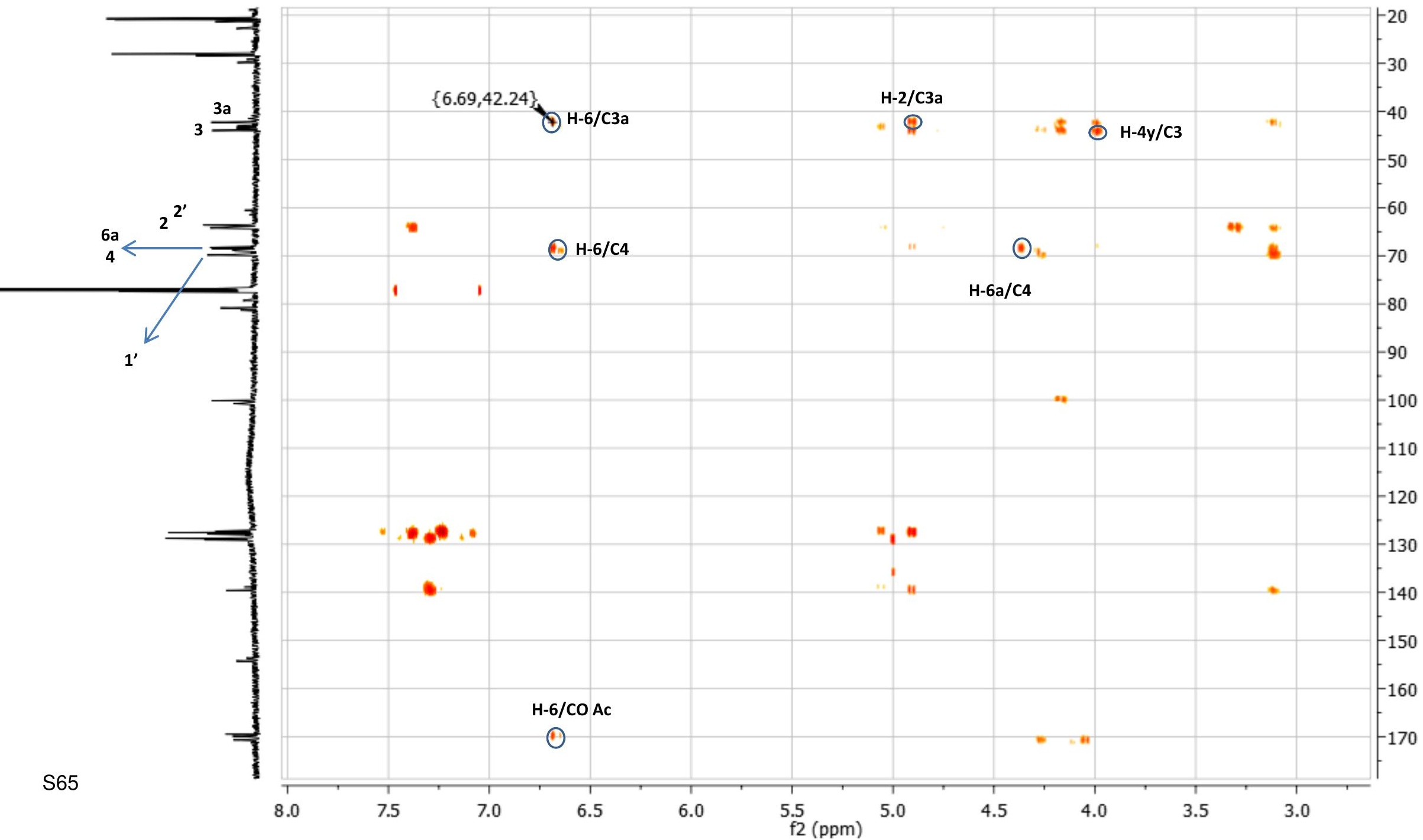

H-4x

H-2'x H-2'y

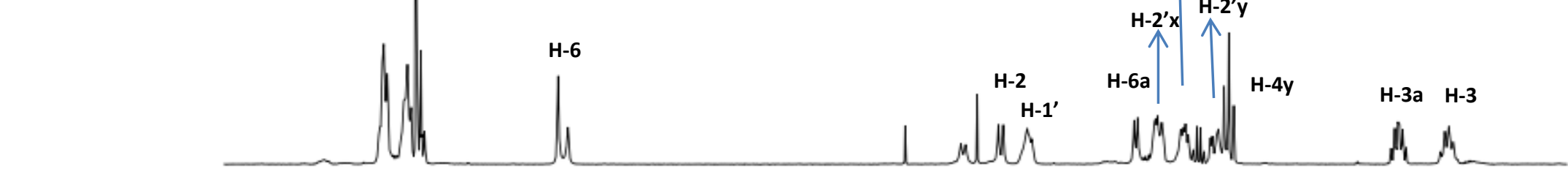


${ }^{1} \mathrm{H}$ NMR (DMSO, $500 \mathrm{MHz}$ ) of compound 12a
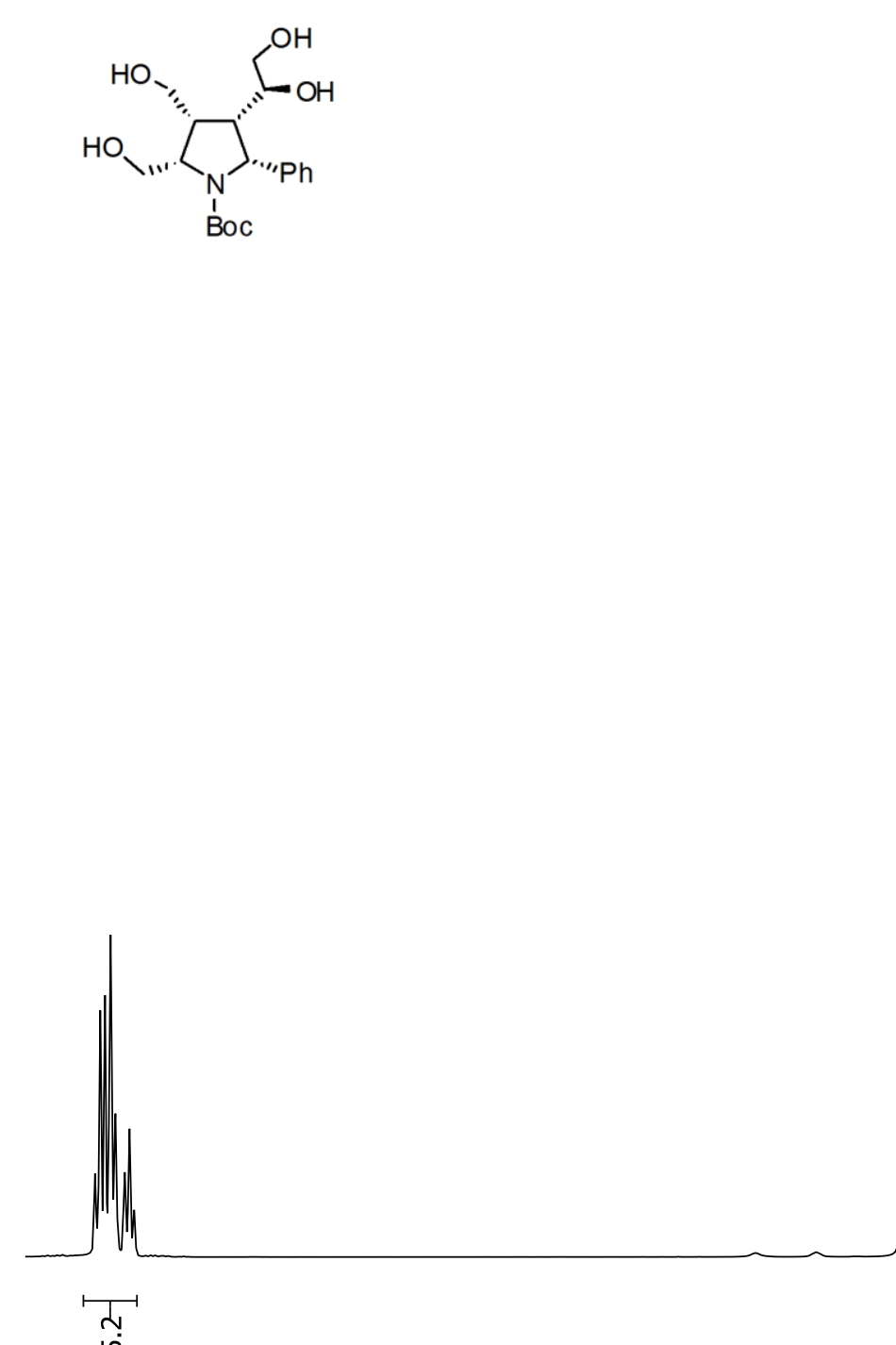

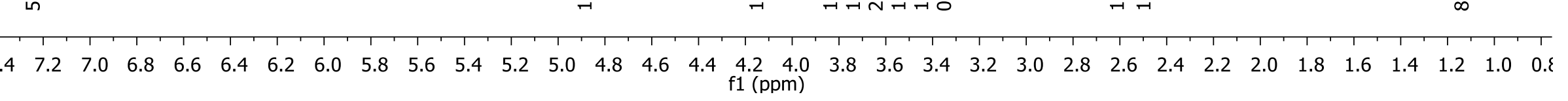


${ }^{13} \mathrm{C}$ NMR (DMSO, 125.7 MHz) of compound 12a

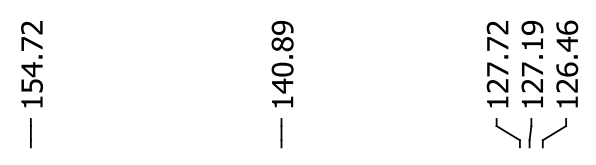

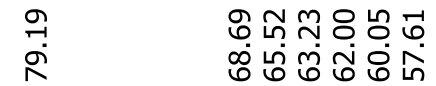

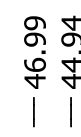

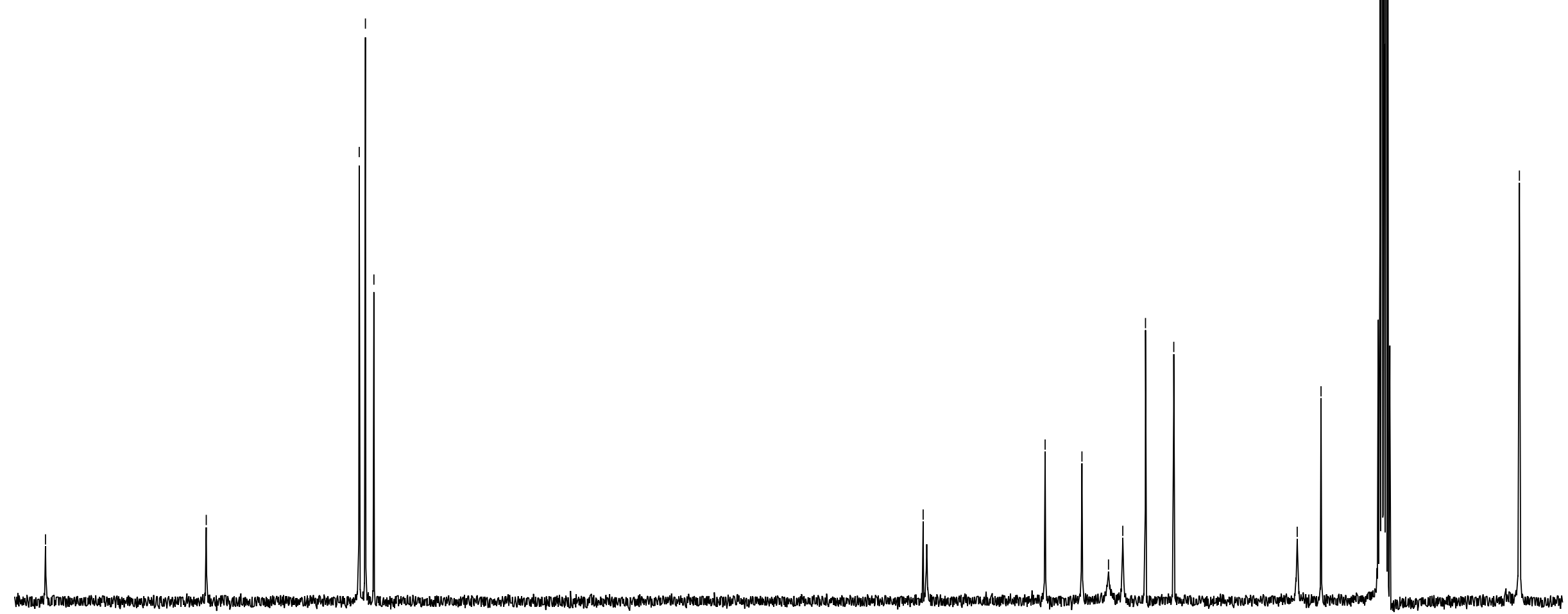


${ }^{1} \mathrm{H} \mathrm{NMR}\left(\mathrm{CDCl}_{3}, 500 \mathrm{MHz}\right)$ of compound $13 \mathbf{a}$
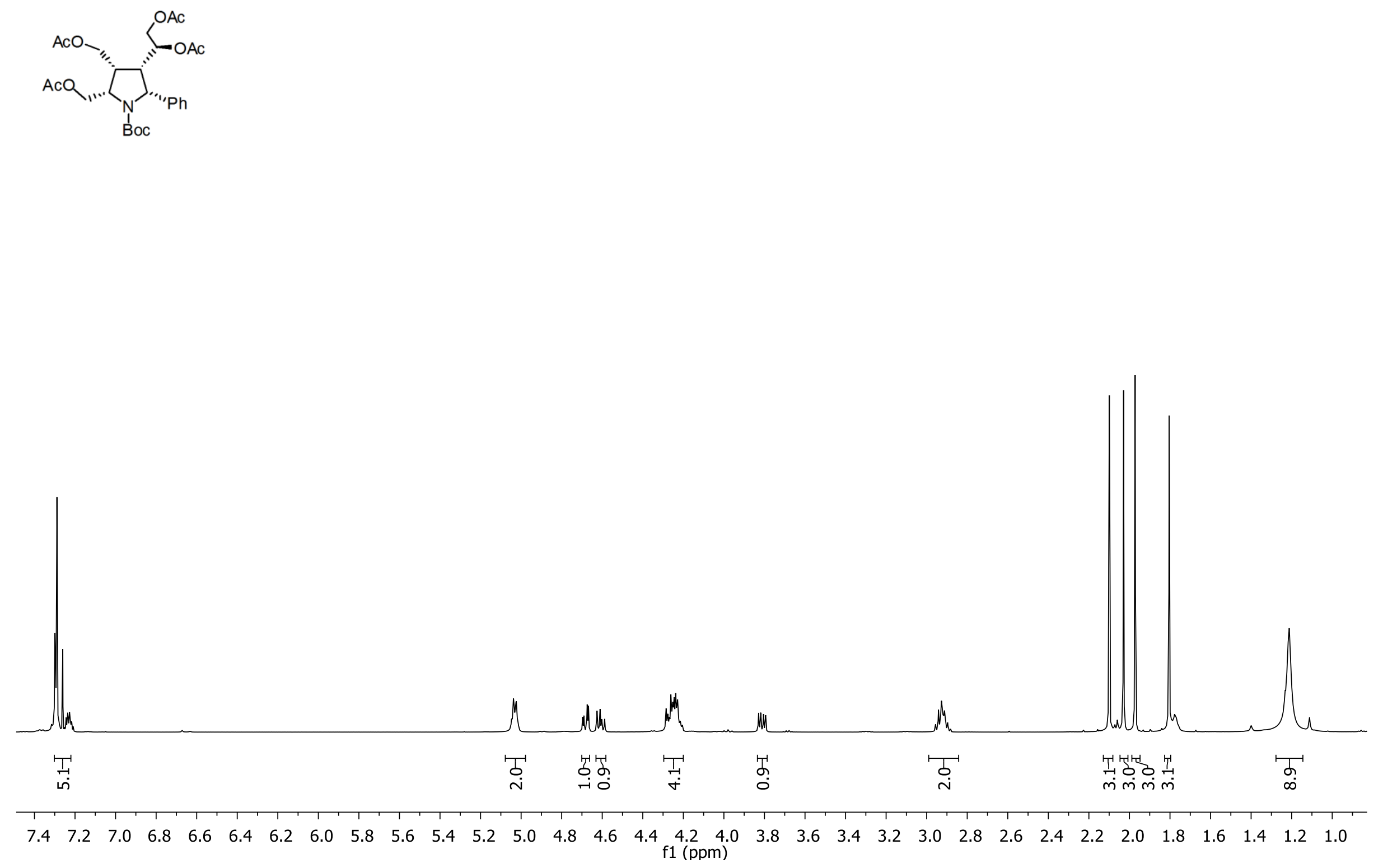
${ }^{13} \mathrm{C} \mathrm{NMR}\left(\mathrm{CDCl}_{3}, 125.7 \mathrm{MHz}\right)$ of compound 13a

\begin{tabular}{|c|c|c|c|c|c|c|c|c|}
\hline 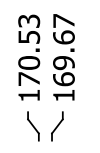 & 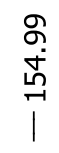 & 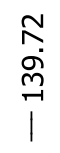 & 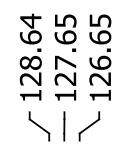 & $\begin{array}{l}N \\
\infty \\
\infty\end{array}$ & 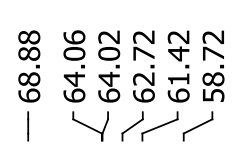 & 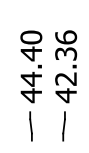 & $\begin{array}{l}\text { O } \\
\stackrel{\infty}{N} \\
1\end{array}$ & 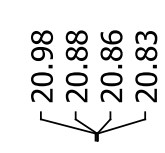 \\
\hline
\end{tabular}

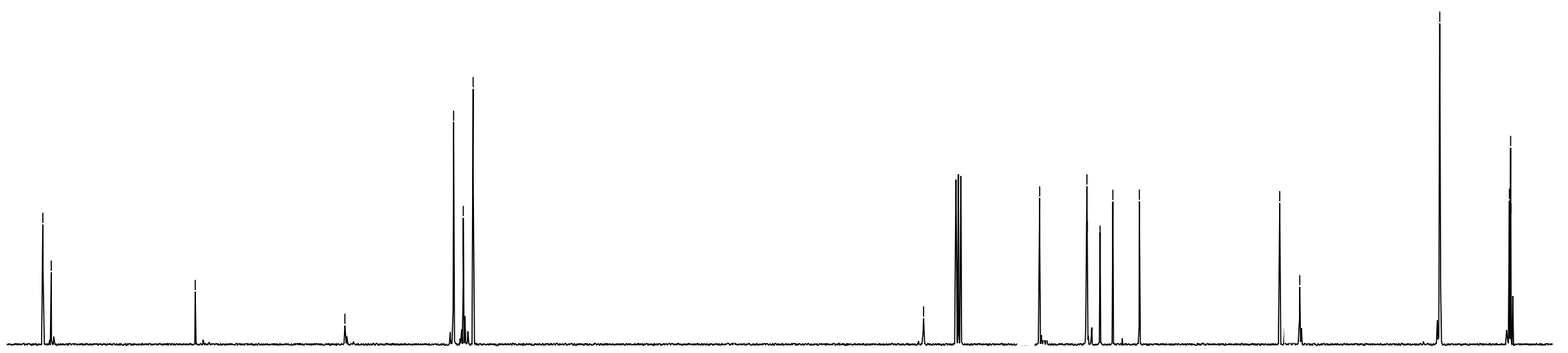

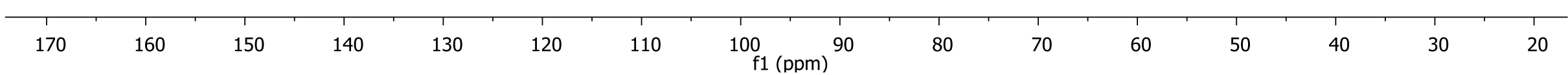




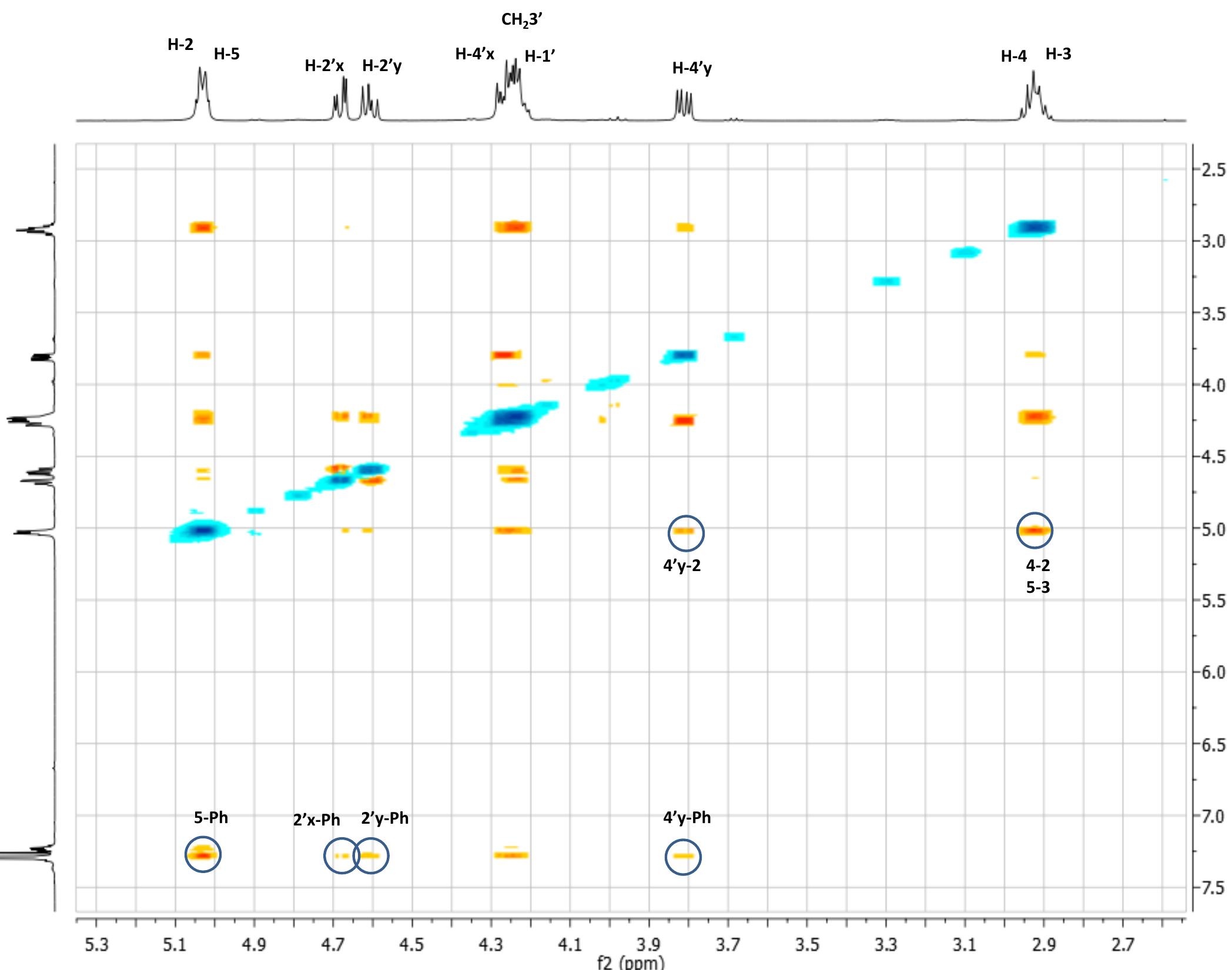

$\overparen{E}$
$\overrightarrow{0}$
$\vec{b}$ 
${ }^{1} \mathrm{H}$ NMR (MeOD, $500 \mathrm{MHz}$ ) of compound 14
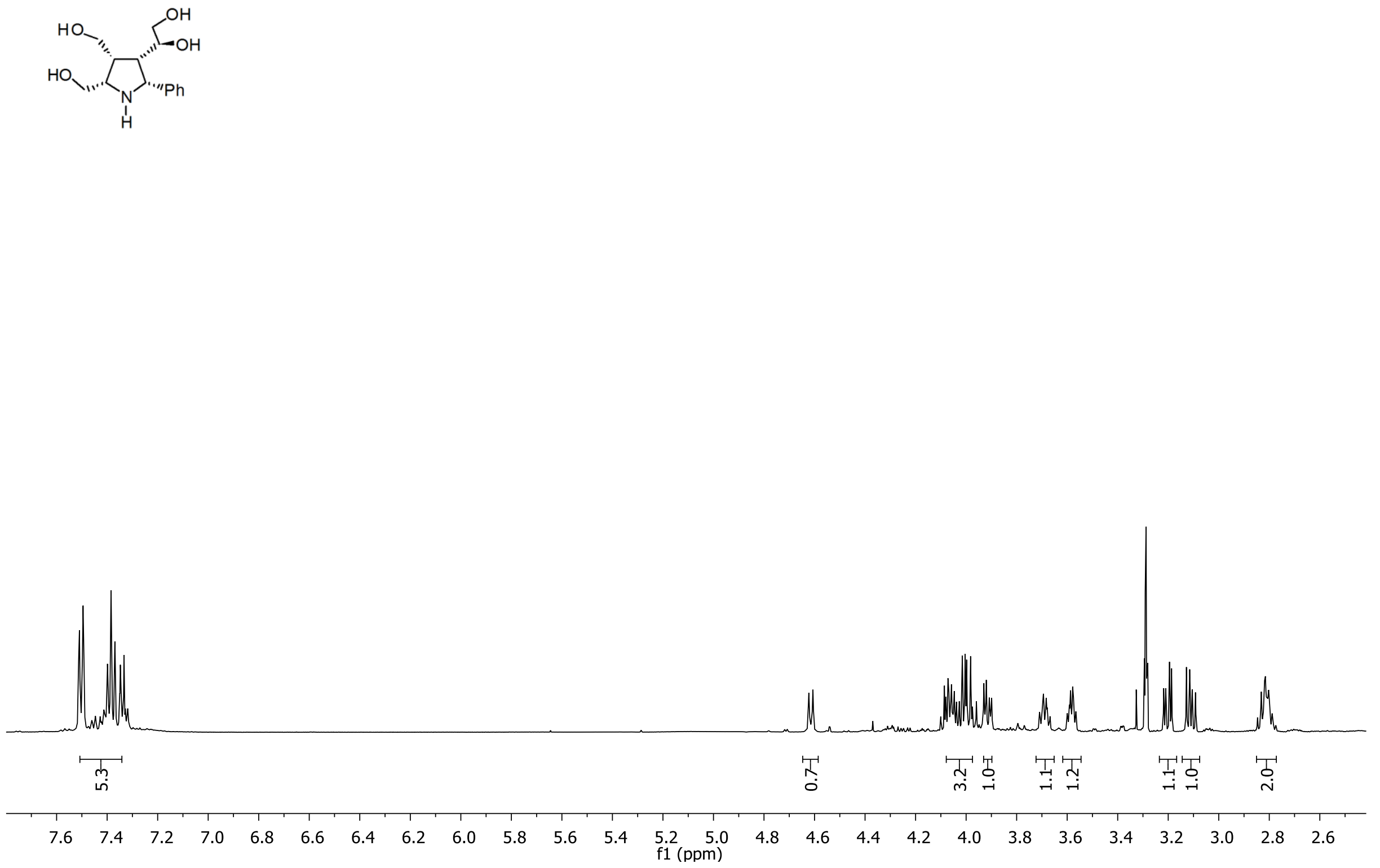
${ }^{13} \mathrm{C}$ NMR (MeOD, $125.7 \mathrm{MHz}$ ) of compound 14
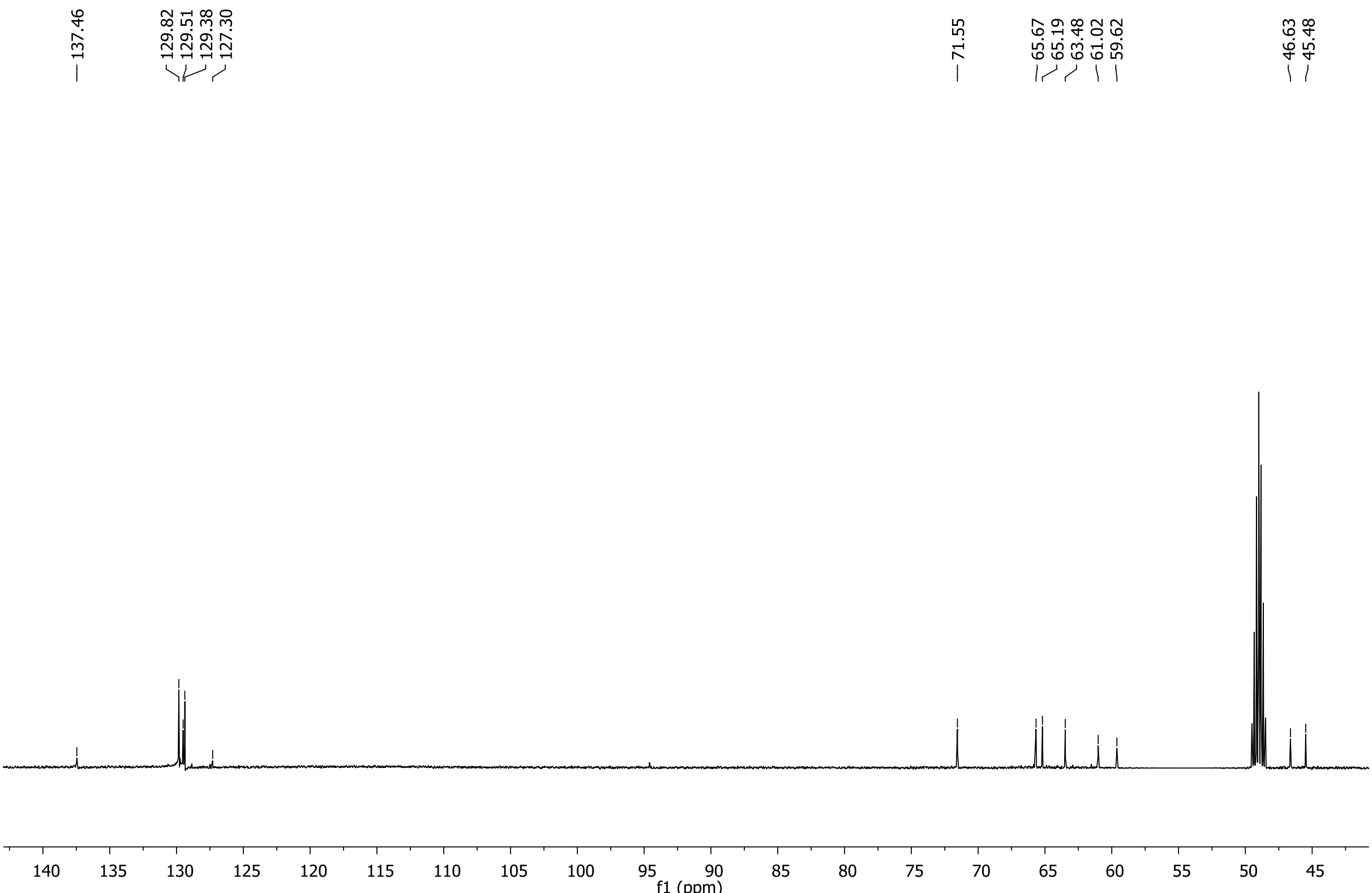
${ }^{1} \mathrm{H}$ NMR $\left(\mathrm{D}_{2} \mathrm{O}, 500 \mathrm{MHz}\right)$ of compound 15a
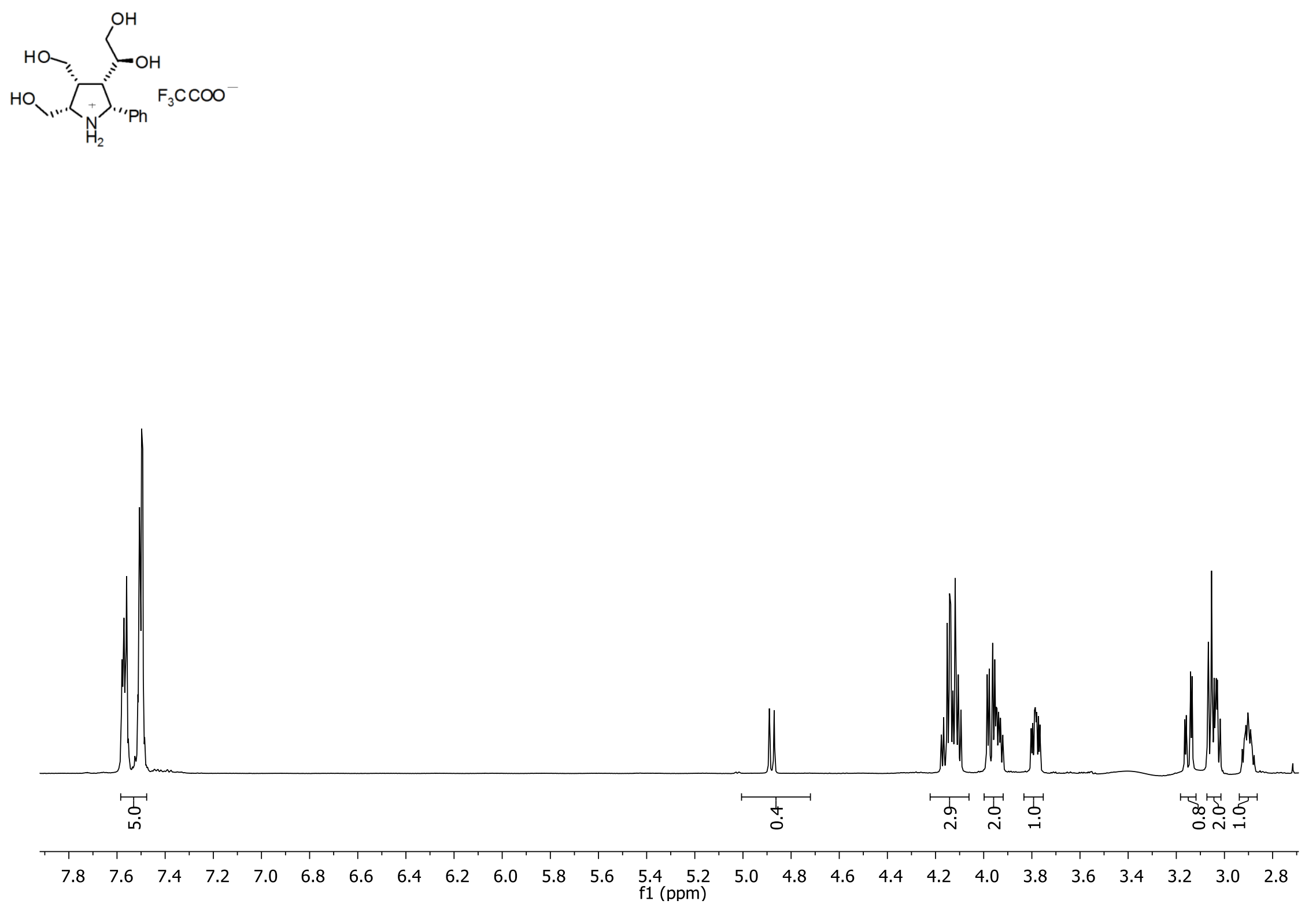
${ }^{13} \mathrm{C}$ NMR $\left(\mathrm{D}_{2} \mathrm{O}, 125.7 \mathrm{MHz}\right)$ of compound 15a

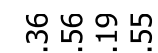

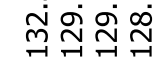

।

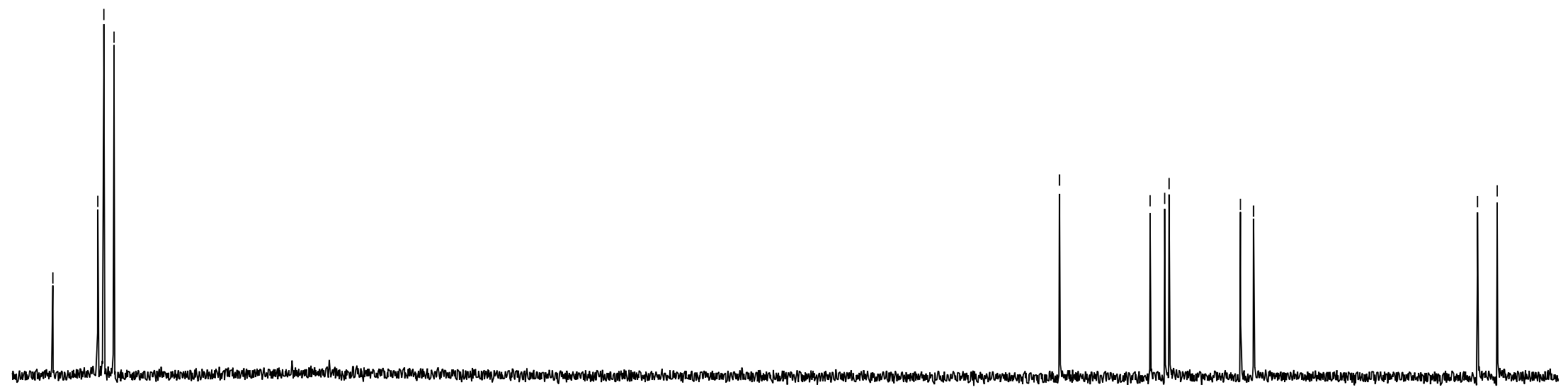


${ }^{1} \mathrm{H} \mathrm{NMR}\left(\mathrm{CDCl}_{3}, 500 \mathrm{MHz}\right)$ of compound 16a
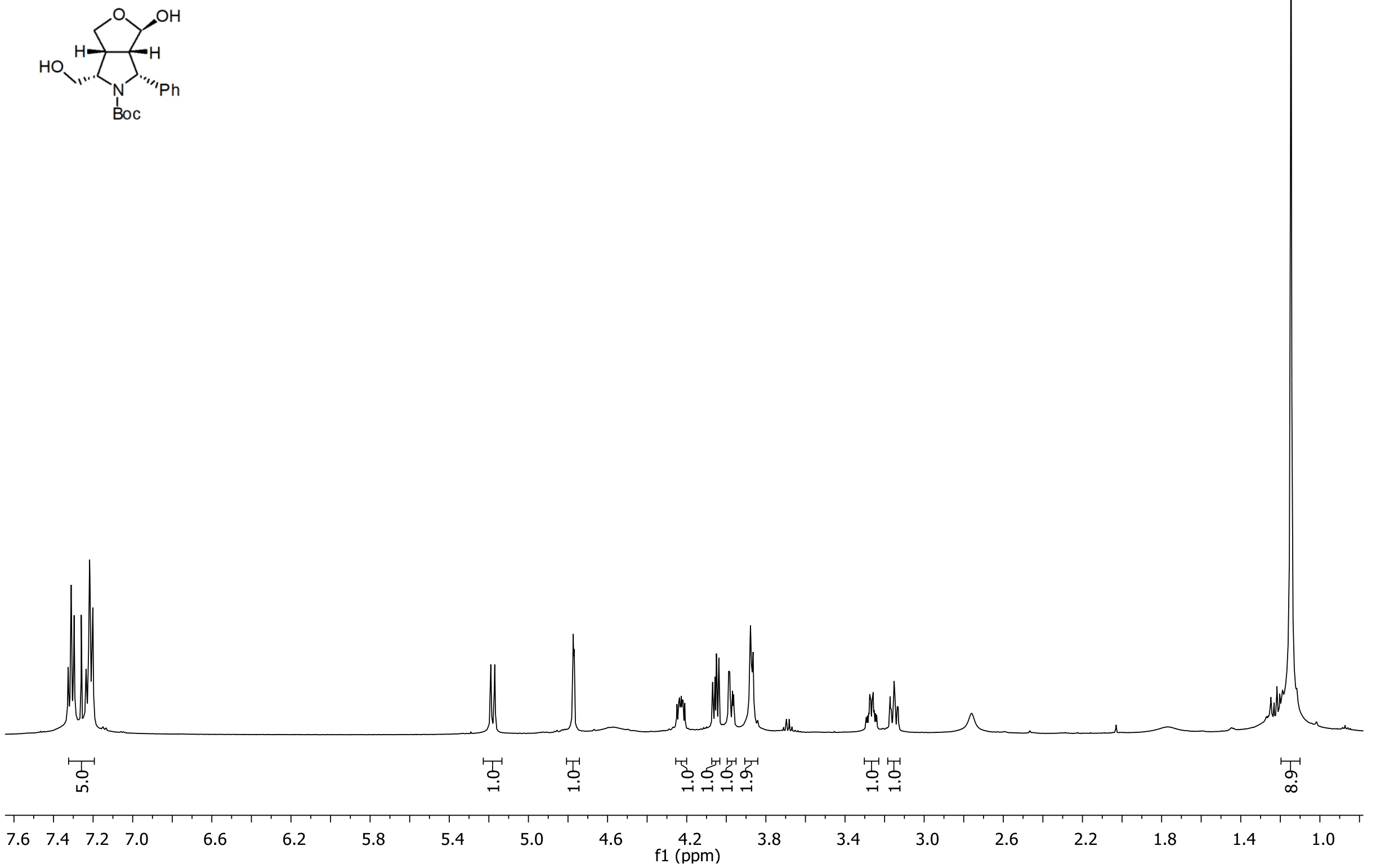
${ }^{3} \mathrm{C} \mathrm{NMR}\left(\mathrm{CDCl}_{3}, 125.7 \mathrm{MHz}\right)$ of compound 16a

\begin{tabular}{|c|c|c|c|c|c|c|c|c|}
\hline & \begin{tabular}{l}
$\stackrel{0}{\circ}$ \\
$\stackrel{0}{+}$ \\
\multirow{1}{|}{}
\end{tabular} & 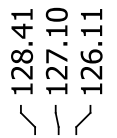 & $\begin{array}{l}\hat{\infty} \\
\dot{\alpha} \\
\text { oे }\end{array}$ & 今. & 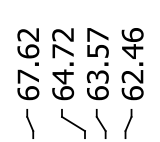 & $\begin{array}{l}\stackrel{8}{0} \\
\text { in } \\
\text { in }\end{array}$ & $\begin{array}{l}\stackrel{+}{m} \\
\stackrel{n}{q}\end{array}$ & $\underset{\substack{o \\
\infty}}{\stackrel{\infty}{1}}$ \\
\hline
\end{tabular}
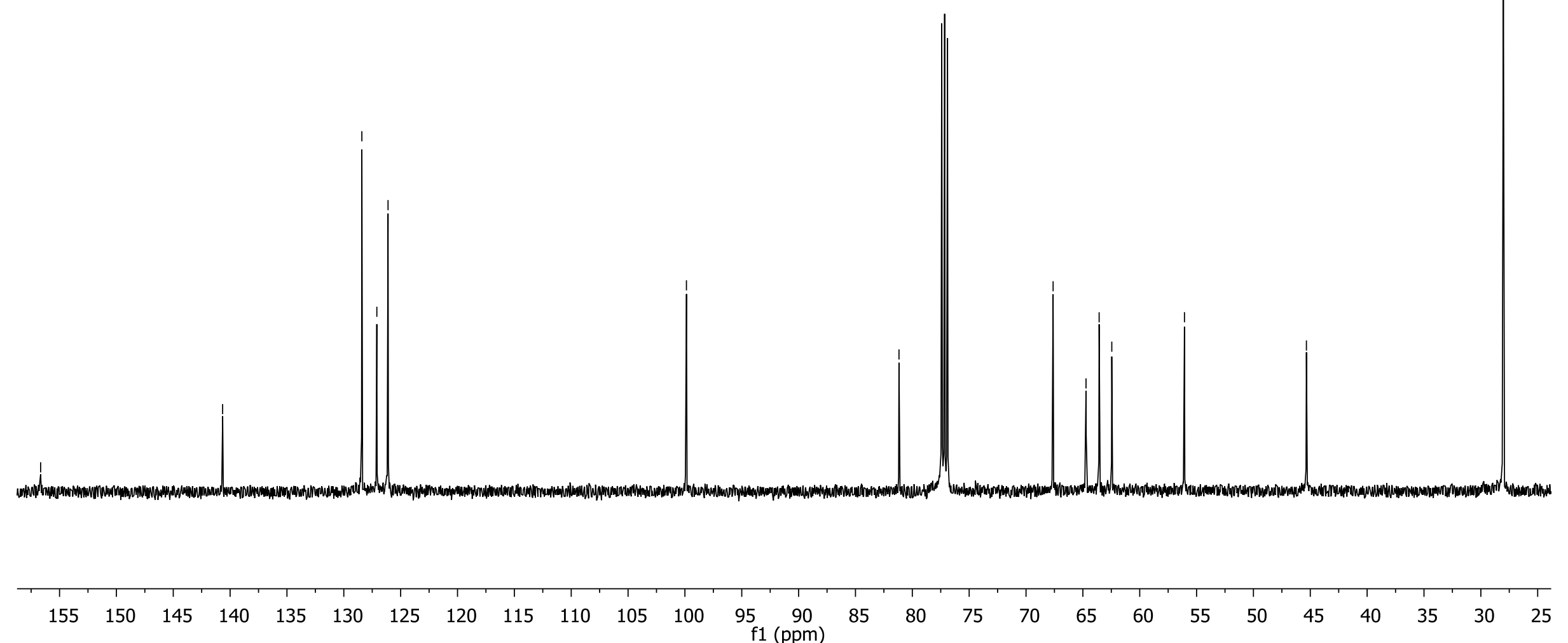


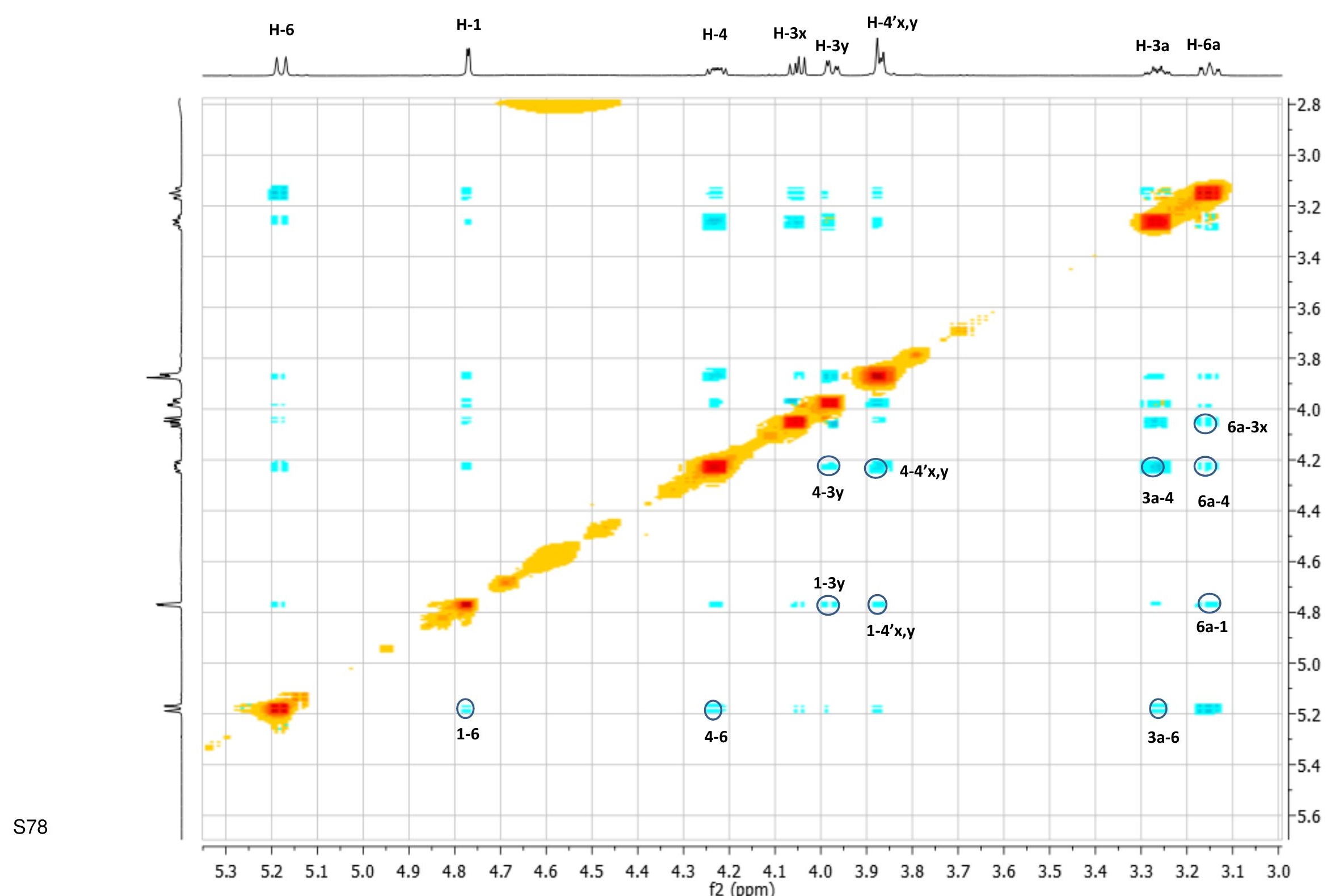




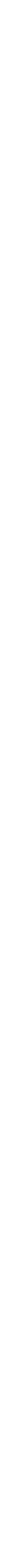


${ }^{1} \mathrm{H}$ NMR $\left(\mathrm{CDCl}_{3}, 500 \mathrm{MHz}\right)$ of compound $17 \mathbf{a}$
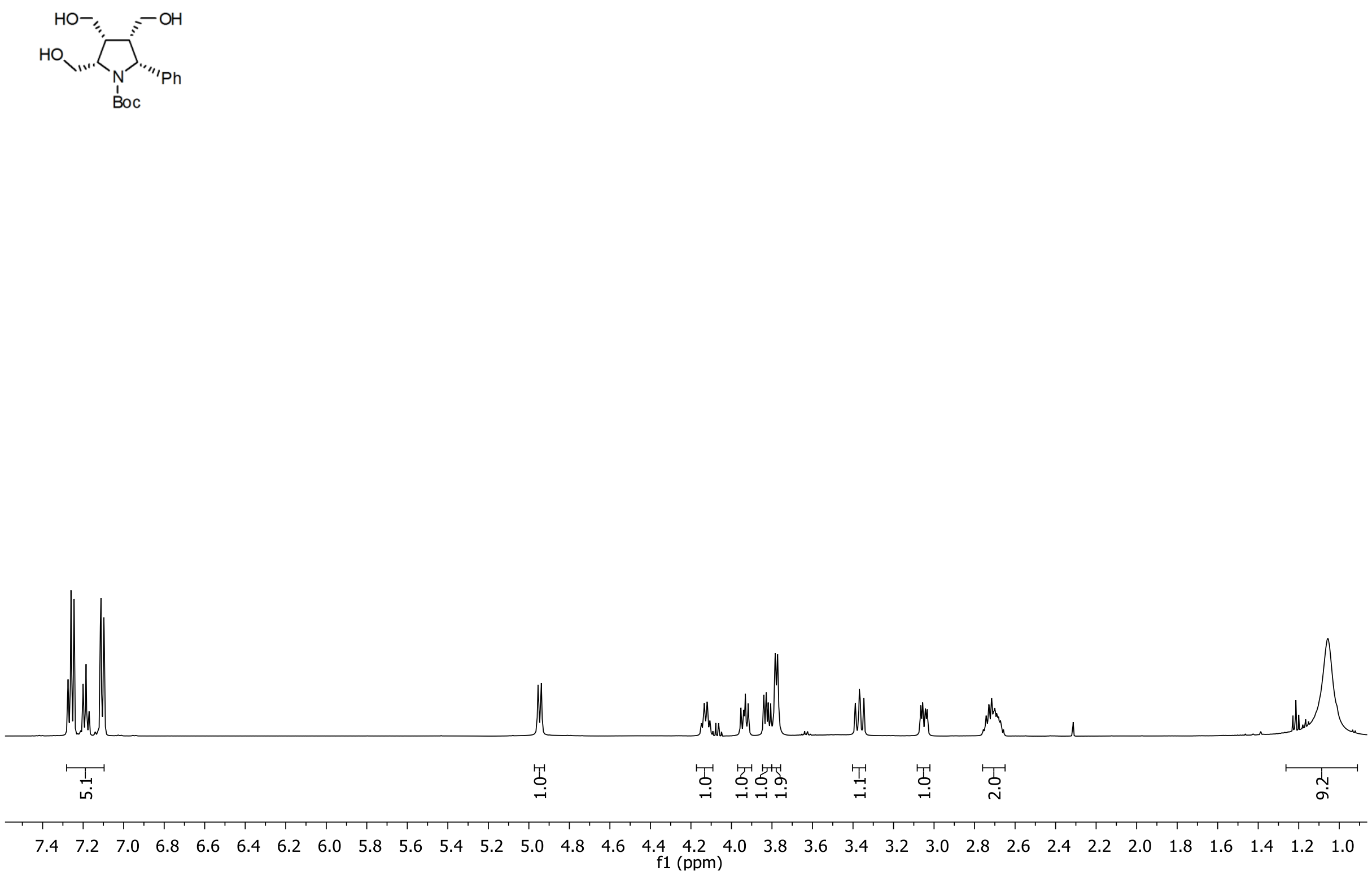
${ }^{13} \mathrm{C} \mathrm{NMR}\left(\mathrm{CDCl}_{3}, 125.7 \mathrm{MHz}\right)$ of compound 17a

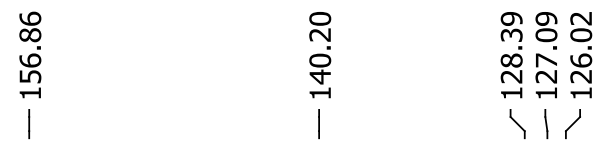

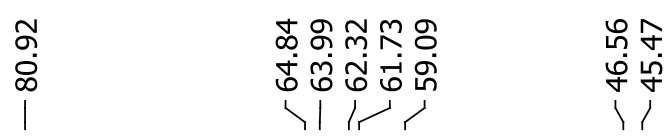

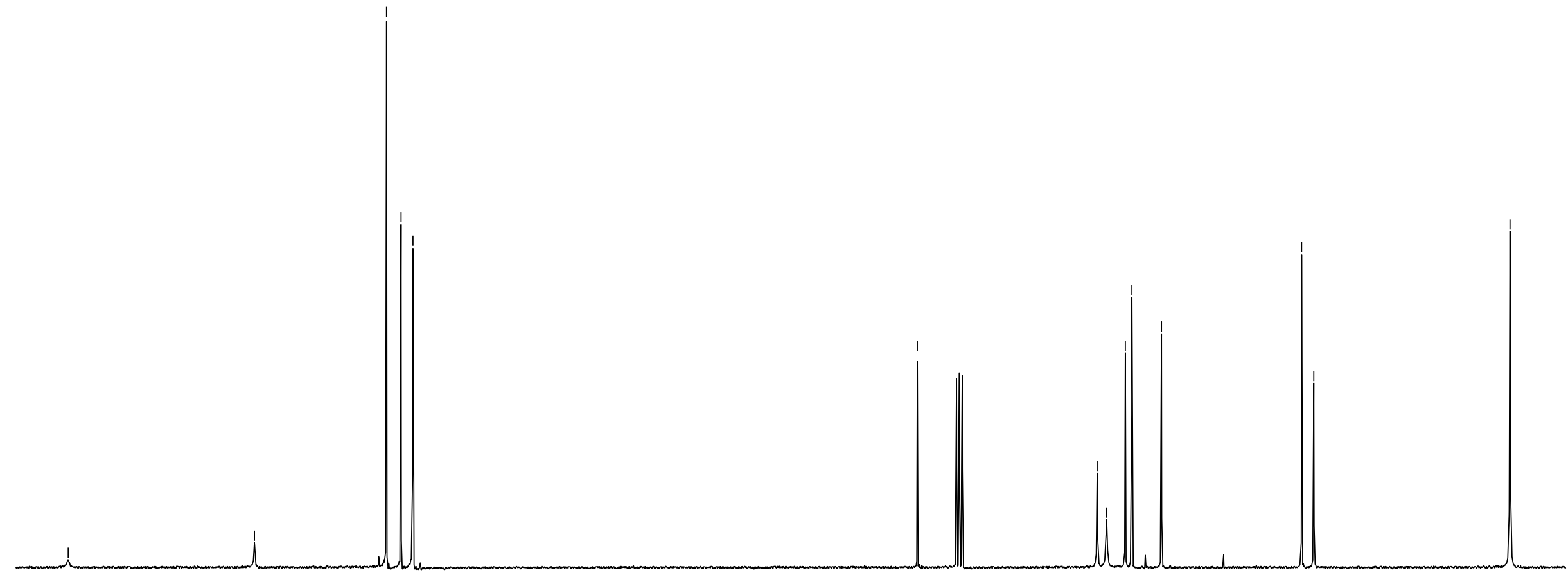

160 


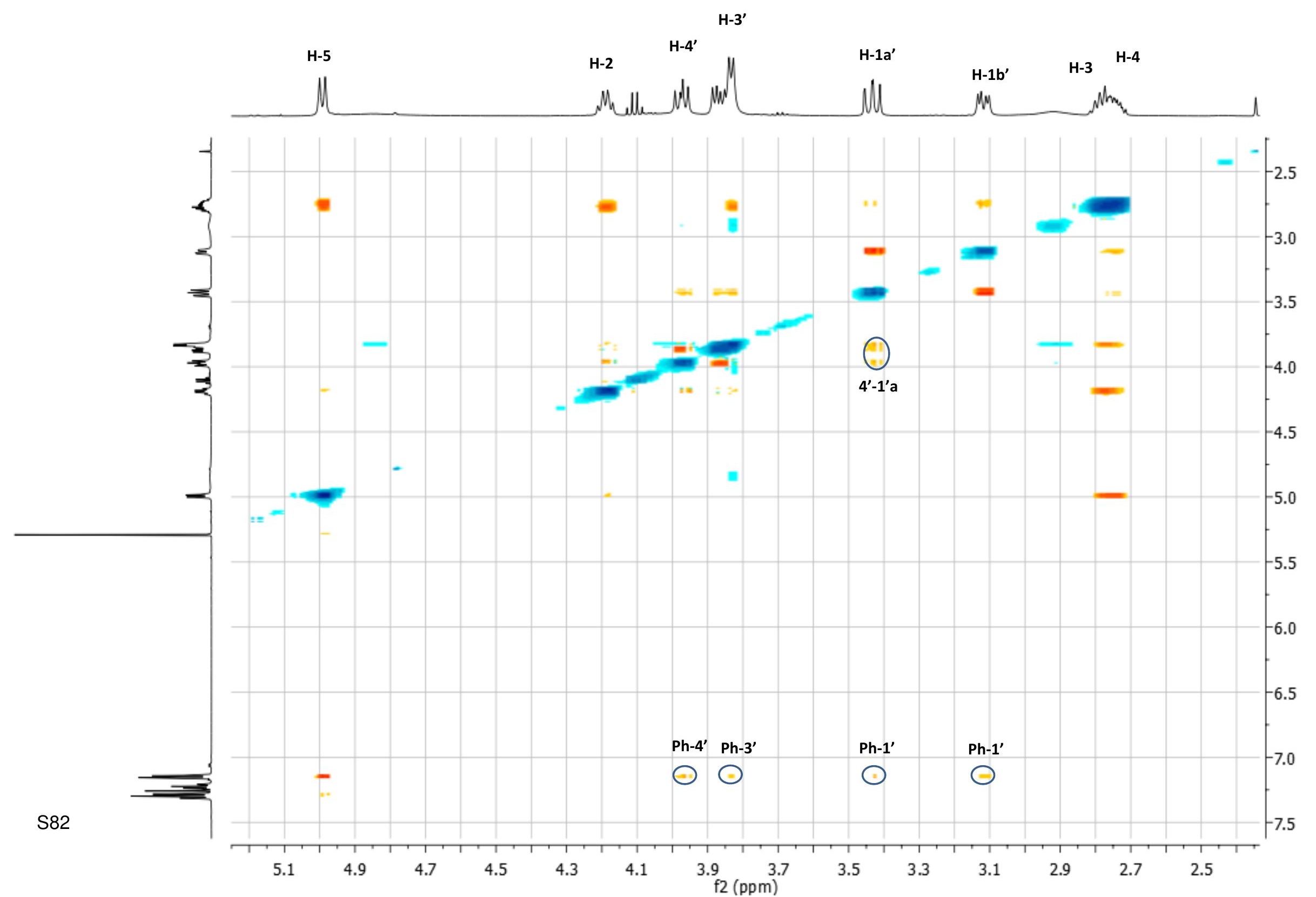


${ }^{1} \mathrm{H}$ NMR $\left(\mathrm{D}_{2} \mathrm{O}, 500 \mathrm{MHz}\right)$ of compound 18a

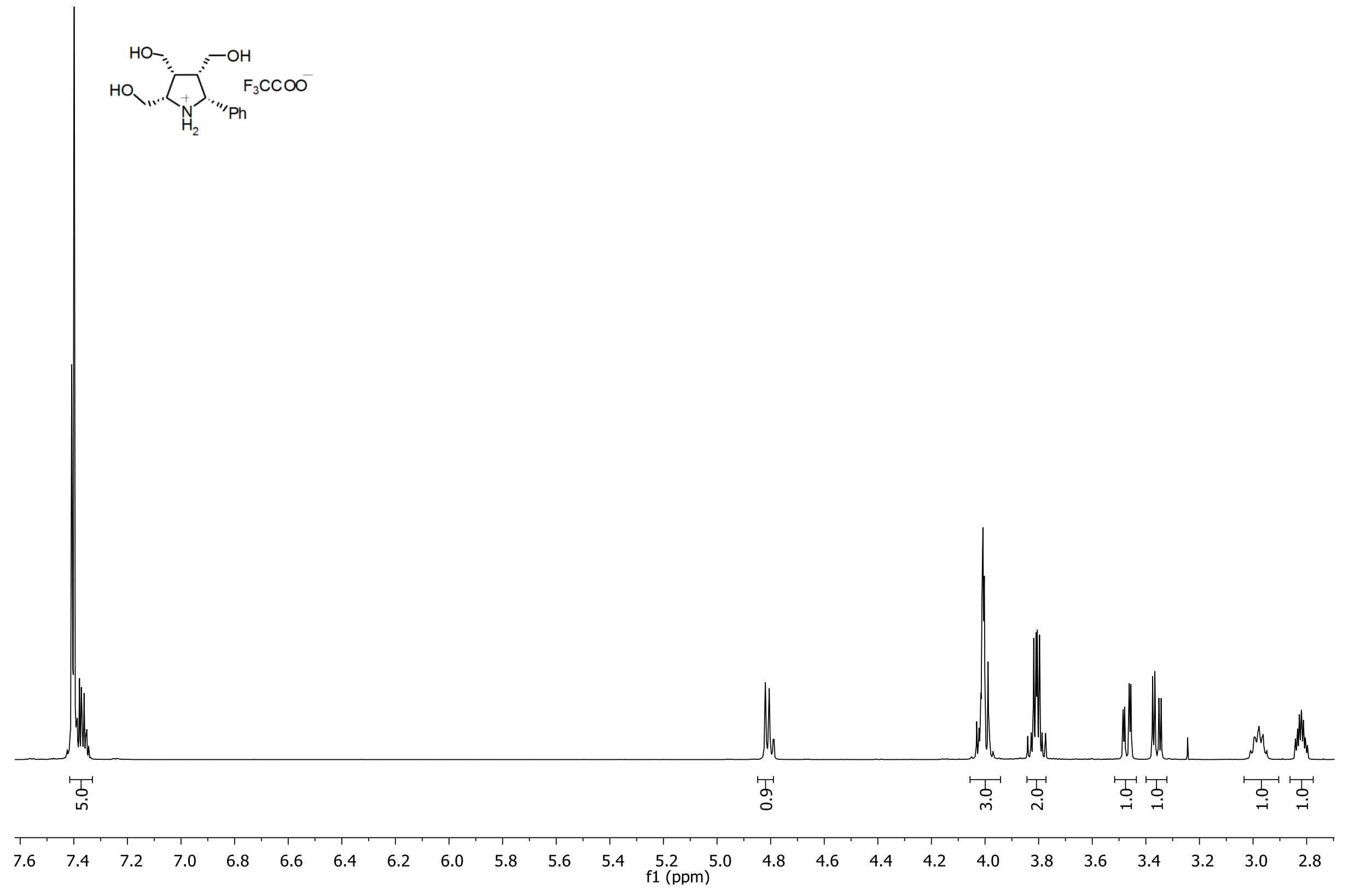


${ }^{1} \mathrm{H}-\mathrm{NMR}$ (pyridine- $d_{5}, 500 \mathrm{MHz}$ ) of compound $\mathbf{6 b}$
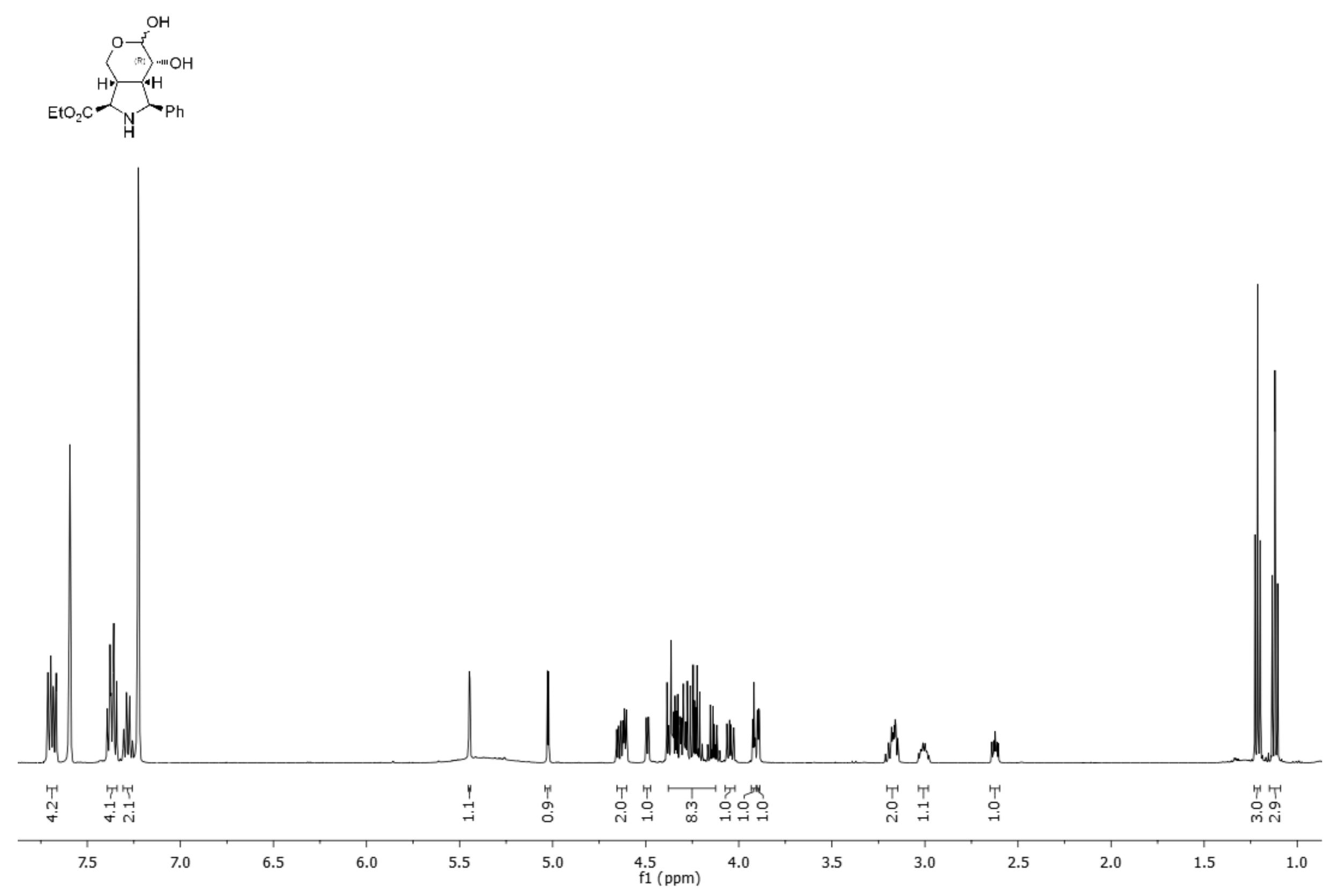
${ }^{13} \mathrm{C}-\mathrm{NMR}$ (pyridine- $d_{5}, 125.7 \mathrm{MHz}$ ) of compound $\mathbf{6 b}$

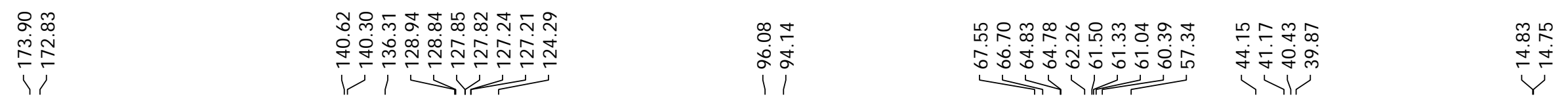

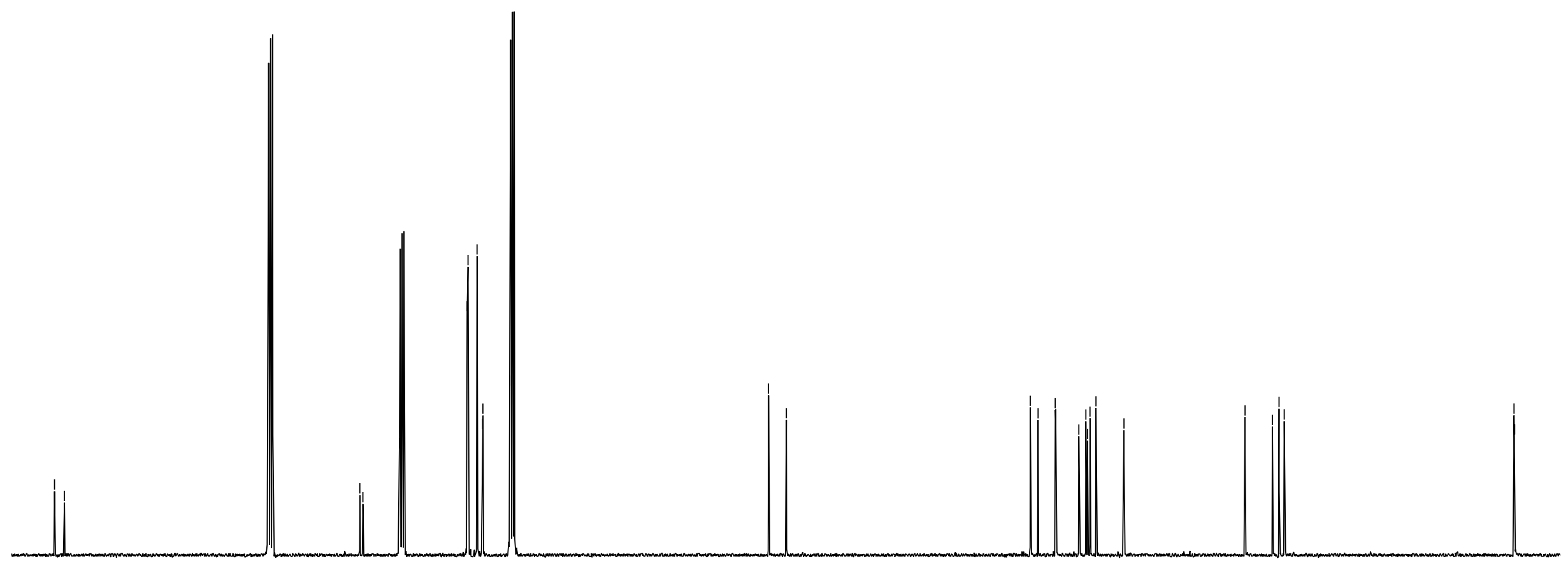


${ }^{1} \mathrm{H}-\mathrm{NMR}\left(\mathrm{CDCl}_{3}, 500 \mathrm{MHz}\right)$ of compound $\mathbf{7 b}$

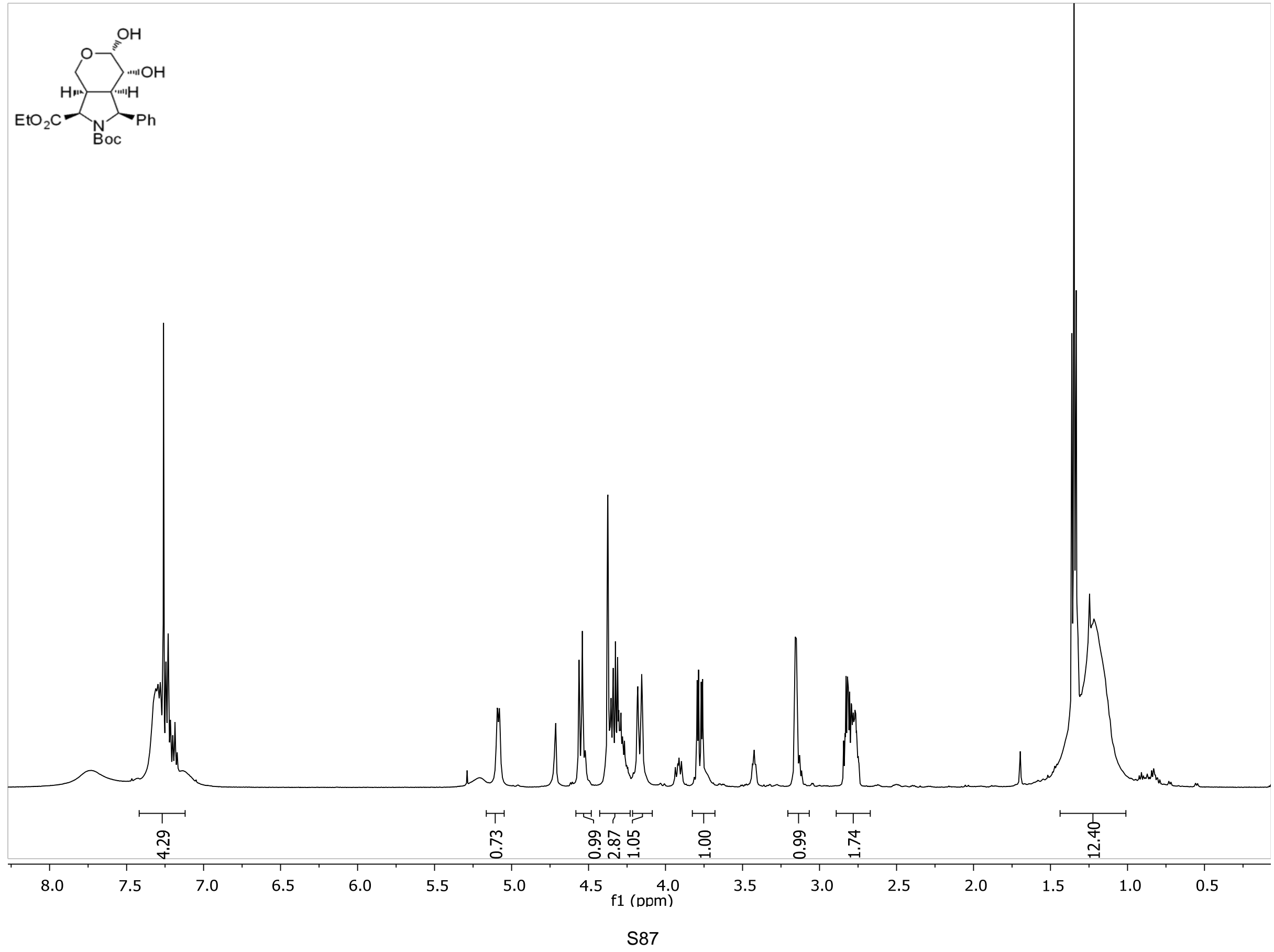


${ }^{13} \mathrm{C}-\mathrm{NMR}\left(\mathrm{CDCl}_{3}, 125.7 \mathrm{MHz}\right)$ of compound $\mathbf{7 b}$

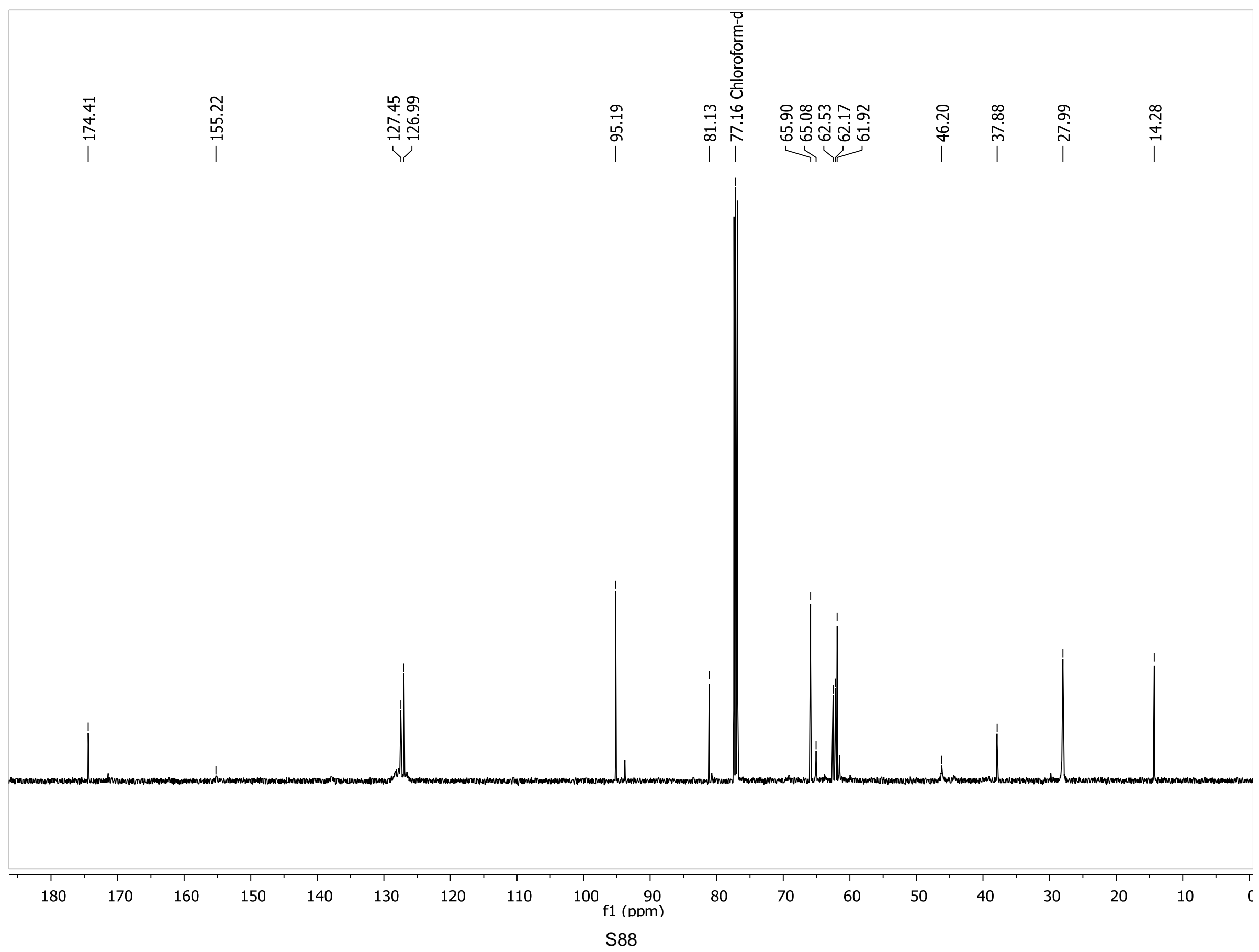


${ }^{1} \mathrm{H}-\mathrm{NMR}\left(\mathrm{CDCl}_{3}, 500 \mathrm{MHz}\right)$ of compound 10b

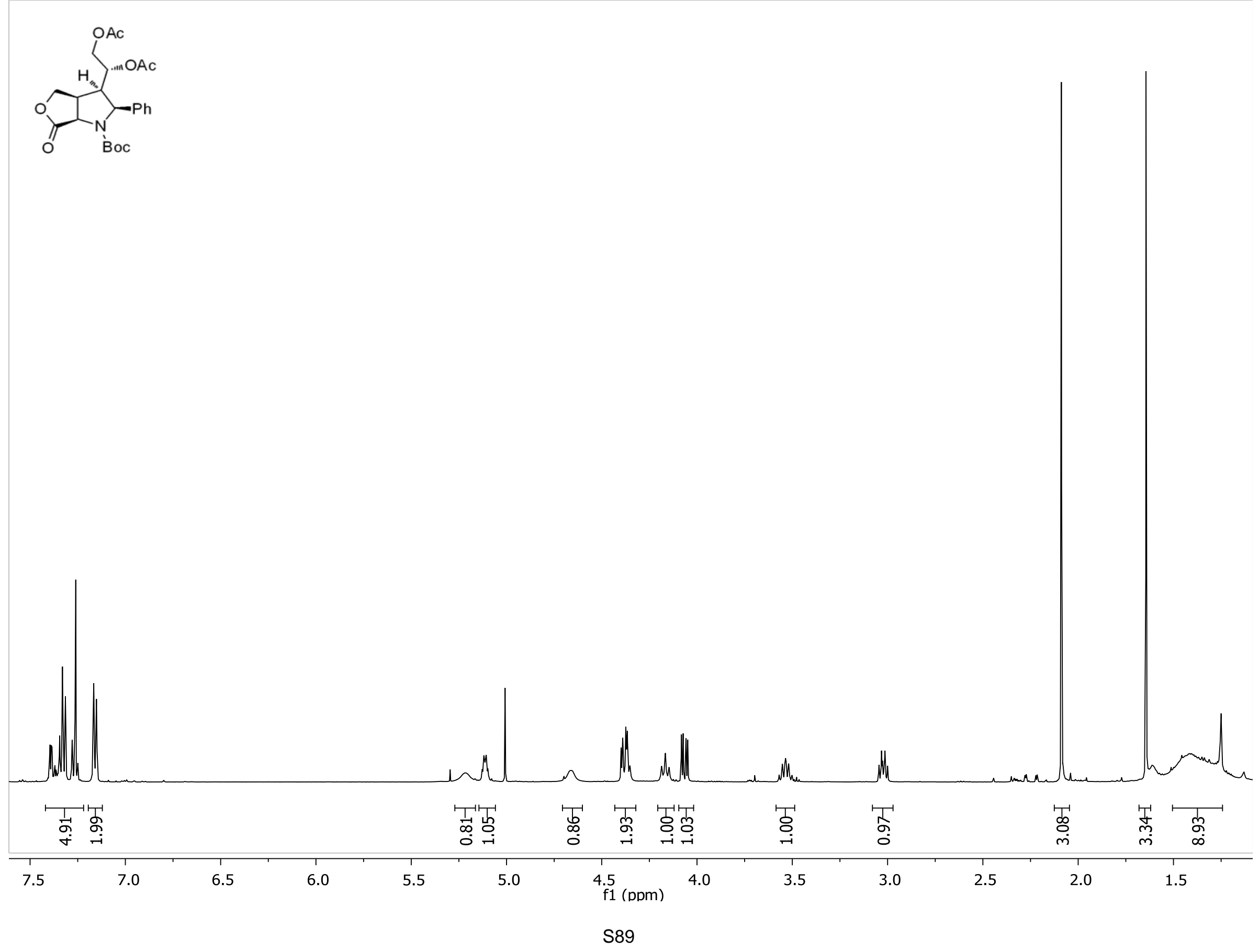


${ }^{13} \mathrm{C}-\mathrm{NMR}\left(\mathrm{CDCl}_{3}, 125.7 \mathrm{MHz}\right)$ of compound 10b

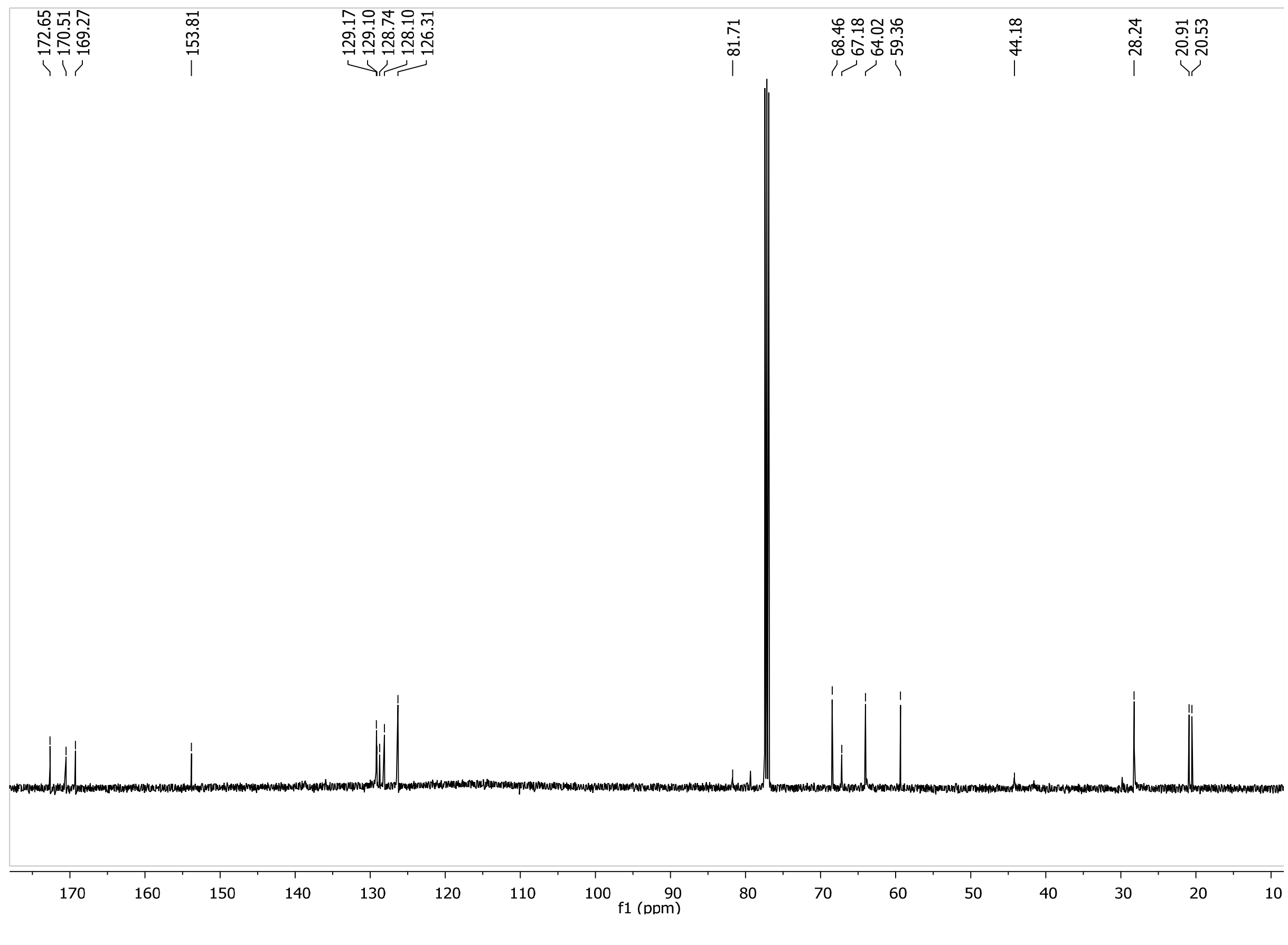


${ }^{1} \mathrm{H}-\mathrm{NMR}\left(\mathrm{CDCl}_{3}, 500 \mathrm{MHz}\right)$ of compound 11b

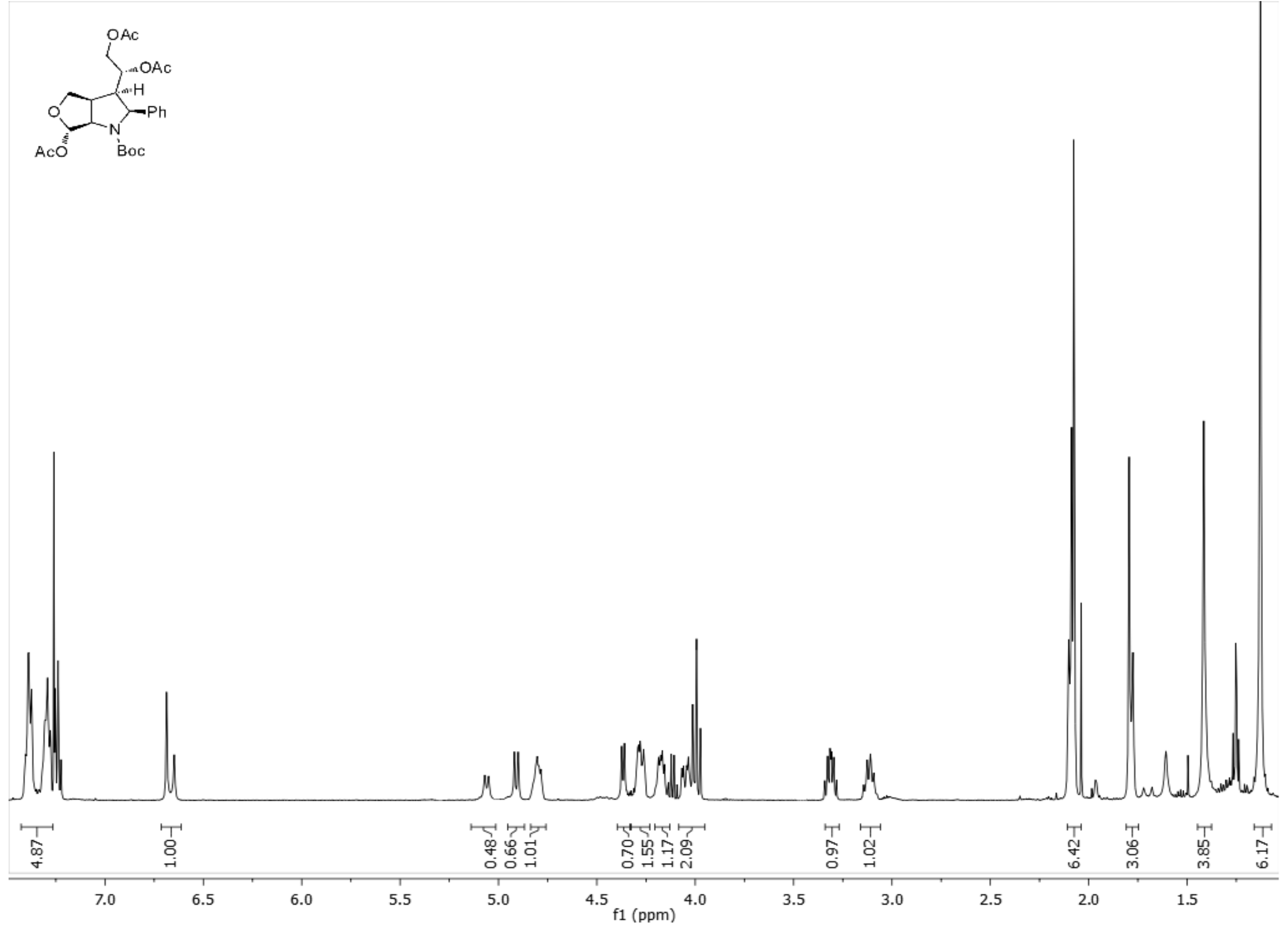


${ }^{13} \mathrm{C}-\mathrm{NMR}\left(\mathrm{CDCl}_{3}, 125.7 \mathrm{MHz}\right)$ of compound 11b

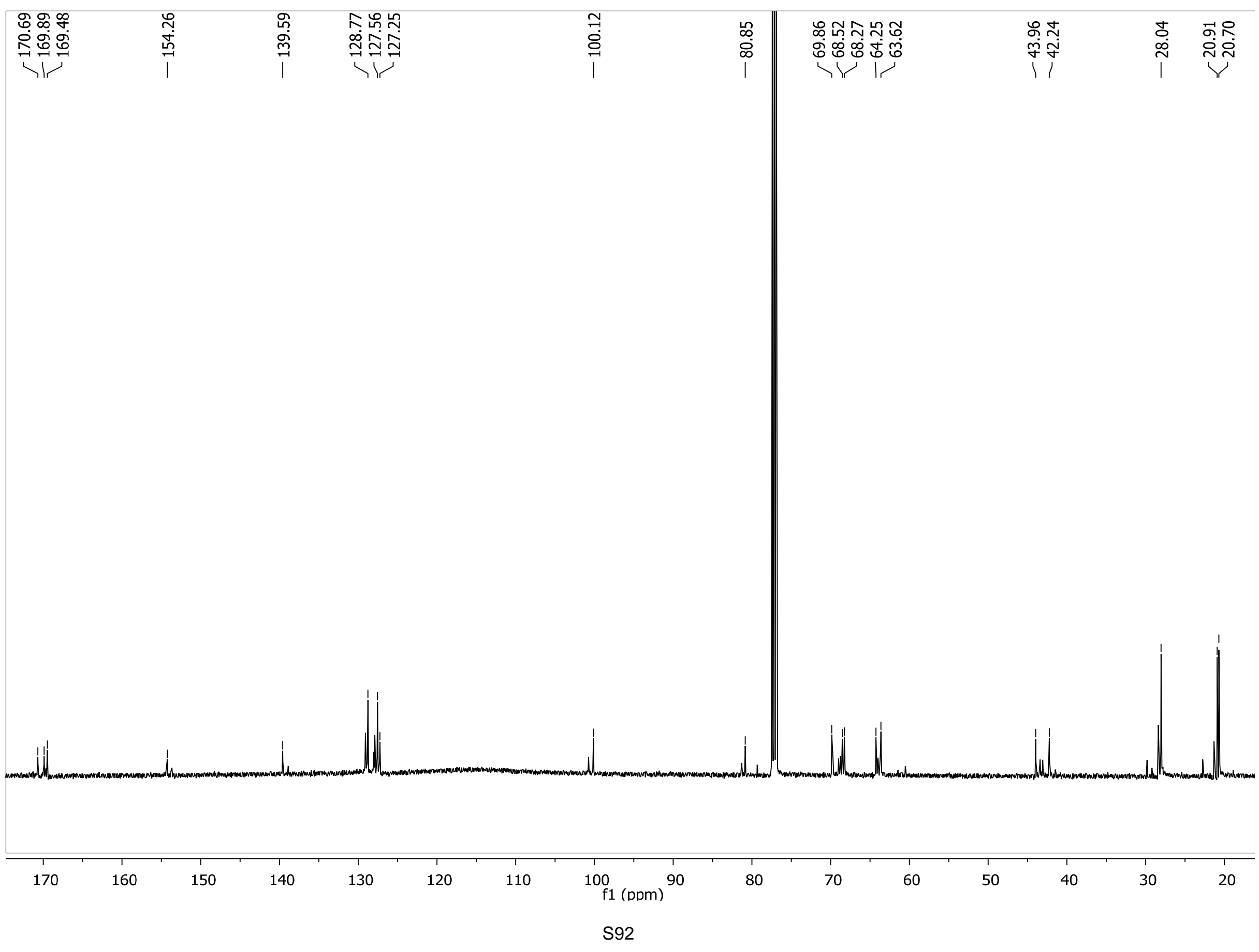


${ }^{1} \mathrm{H}-\mathrm{NMR}$ (DMSO, $500 \mathrm{MHz}$ ) of compound 12b
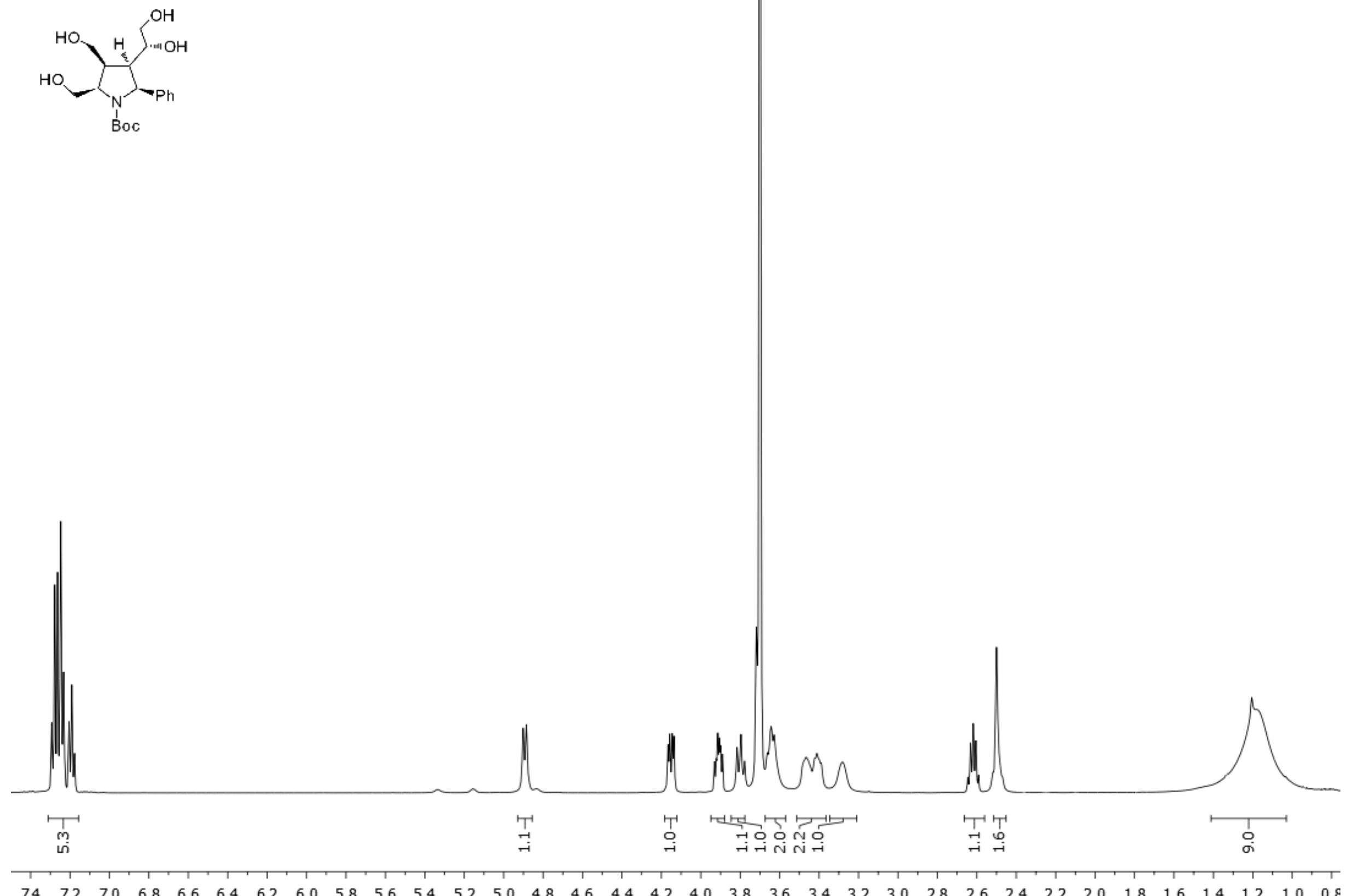

$\begin{array}{llllllllllllllllllllllllllllllllll}7.4 & 7.2 & 7.0 & 6.8 & 6.6 & 6.4 & 6.2 & 6.0 & 5.8 & 5.6 & 5.4 & 5.2 & 5.0 & 4.8 & 4.6 & 4.4 & 4.2 & 4.0 & 3.8 & 3.6 & 3.4 & 3.2 & 3.0 & 2.8 & 2.6 & 2.4 & 2.2 & 2.0 & 1.8 & 1.6 & 1.4 & 1.2 & 1.0 & 0 . \varepsilon\end{array}$ 
${ }^{13} \mathrm{C}-\mathrm{NMR}$ (DMSO, 125.7 MHz) of compound 12b

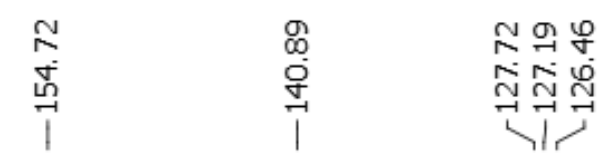

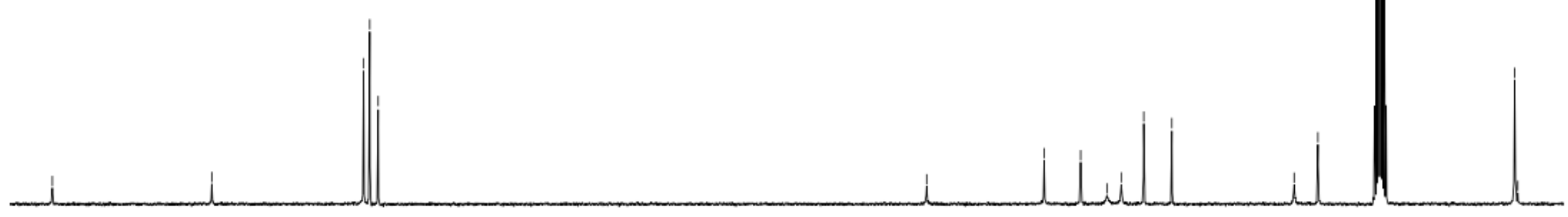


${ }^{1} \mathrm{H}-\mathrm{NMR}\left(\mathrm{CDCl}_{3}, 500 \mathrm{MHz}\right)$ of compound $\mathbf{1 3 b}$
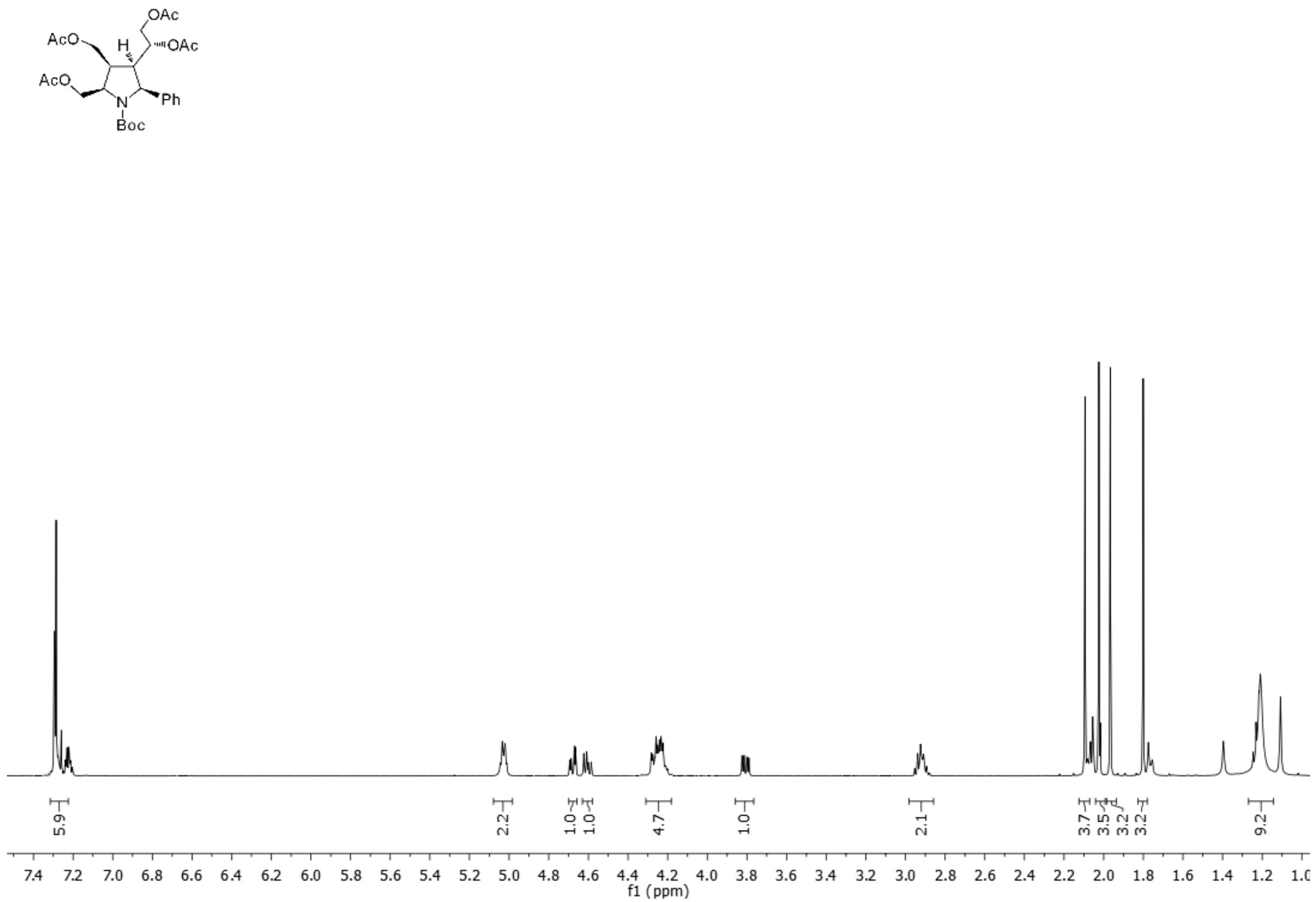
${ }^{13} \mathrm{C}-\mathrm{NMR}\left(\mathrm{CDCl}_{3}, 125.7 \mathrm{MHz}\right)$ of compound $\mathbf{1 3 b}$

\begin{tabular}{|c|}
\hline 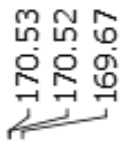 \\
\hline
\end{tabular}

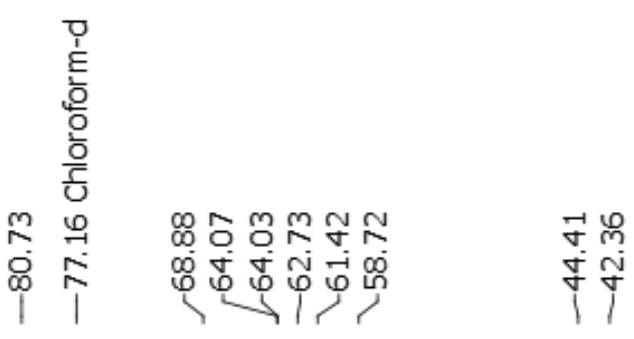

ஜำ

ขึ ํํำ
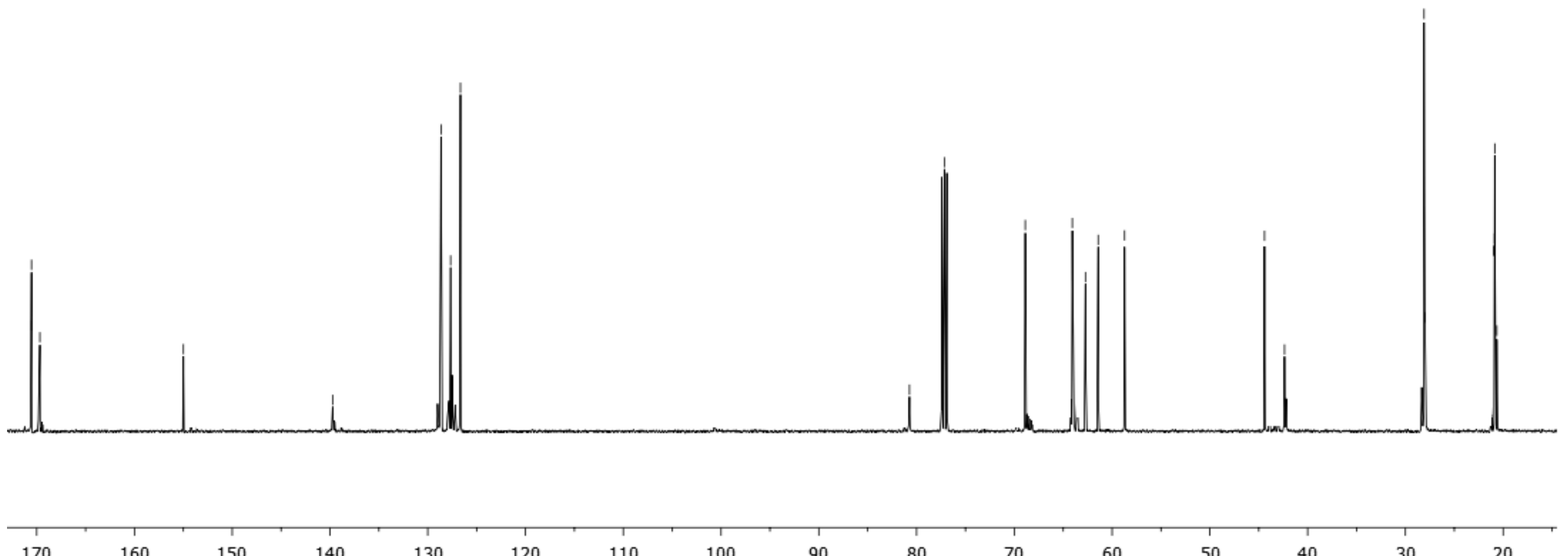

160

150

140

130

120

110

100

$\mathrm{f} 1(\mathrm{ppm})$

80

70

60

50

40

30

20 
${ }^{1} \mathrm{H}-\mathrm{NMR}\left(\mathrm{D}_{2} \mathrm{O}, 500 \mathrm{MHz}\right)$ of compound $\mathbf{1 5 b}$
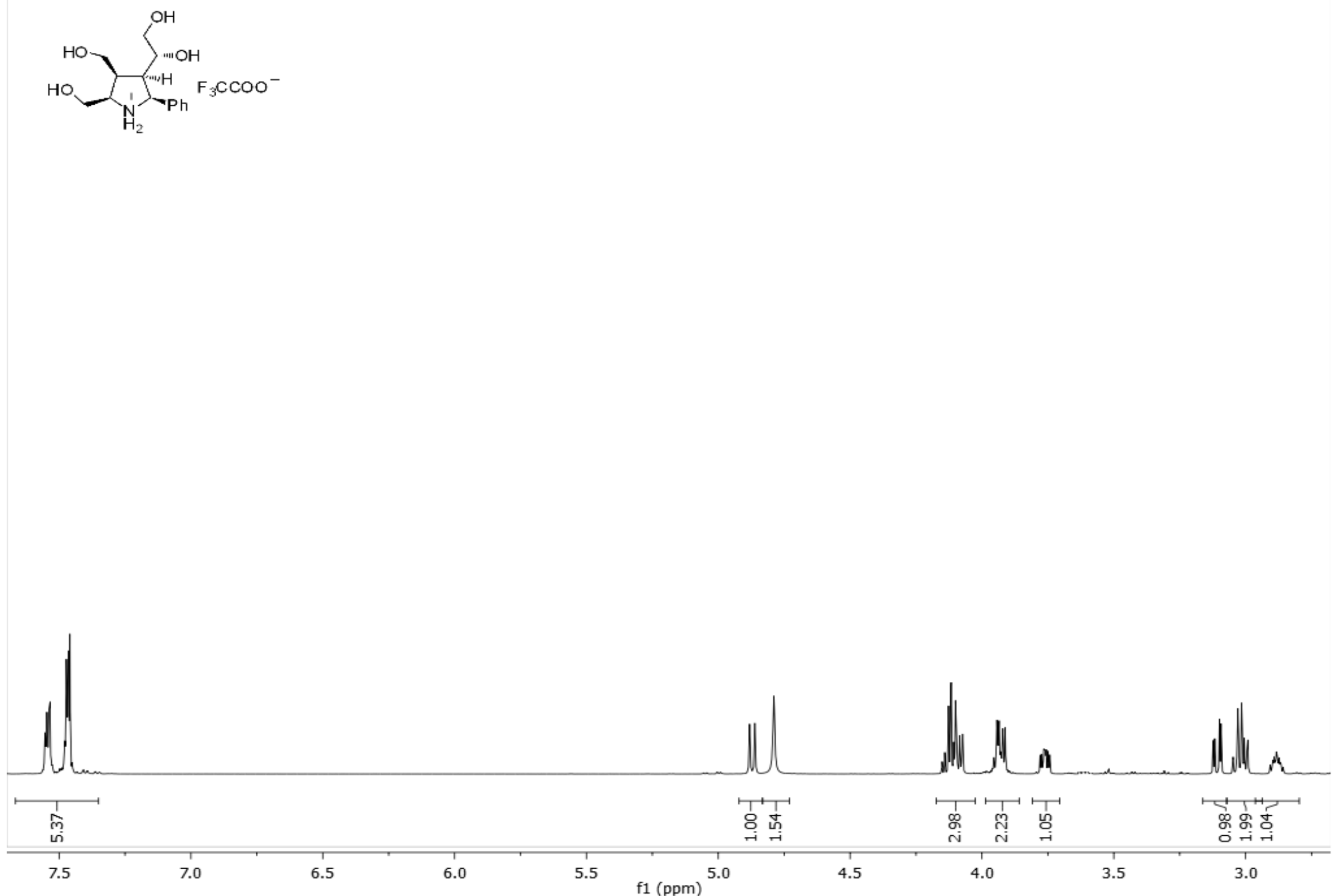
${ }^{13} \mathrm{C}-\mathrm{NMR}\left(\mathrm{D}_{2} \mathrm{O}, 125.7 \mathrm{MHz}\right)$ of compound $\mathbf{1 5 b}$

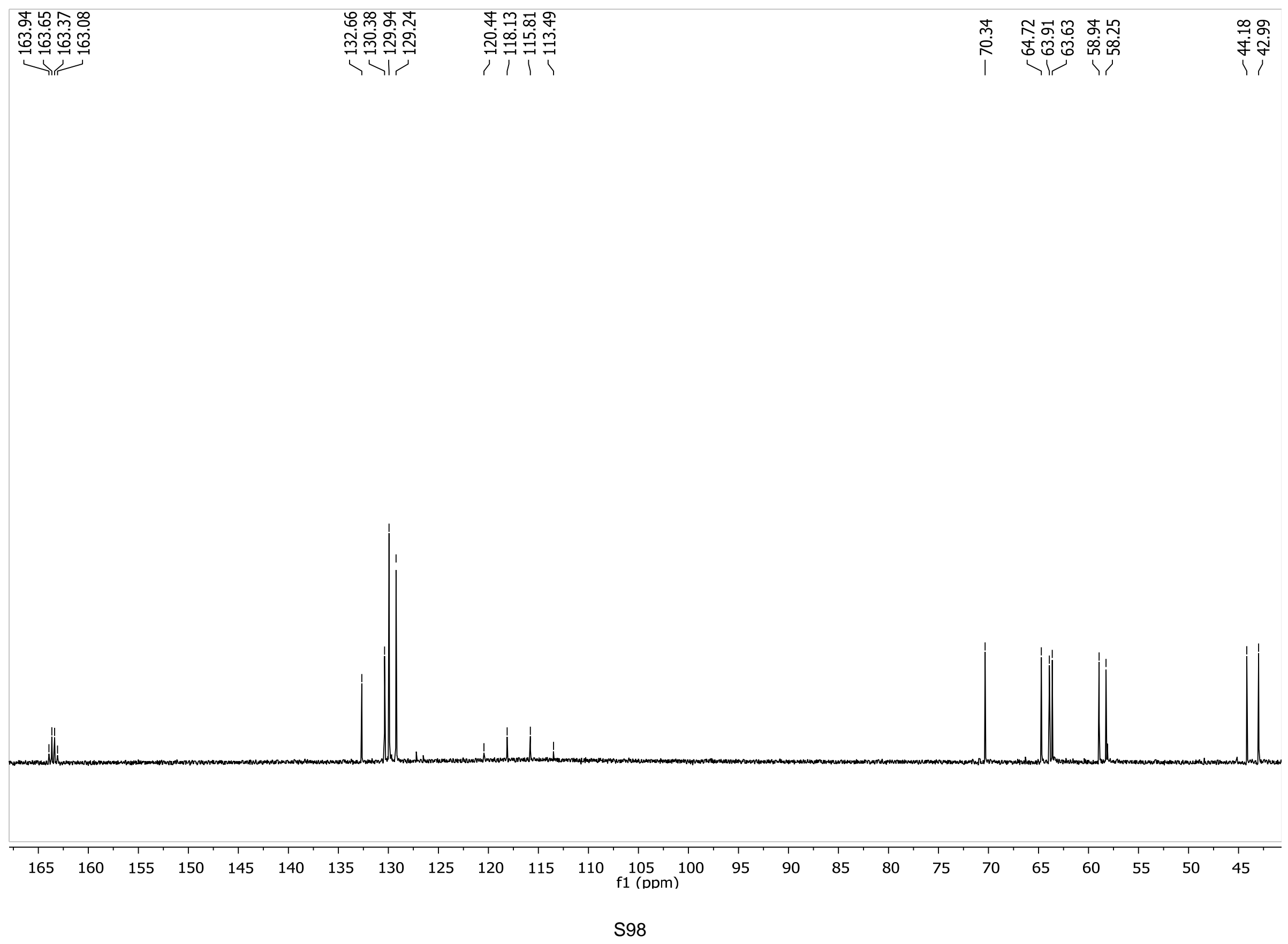


${ }^{1} \mathrm{H}-\mathrm{NMR}\left(\mathrm{CDCl}_{3}, 500 \mathrm{MHz}\right)$ of compound $\mathbf{1 6 b}$
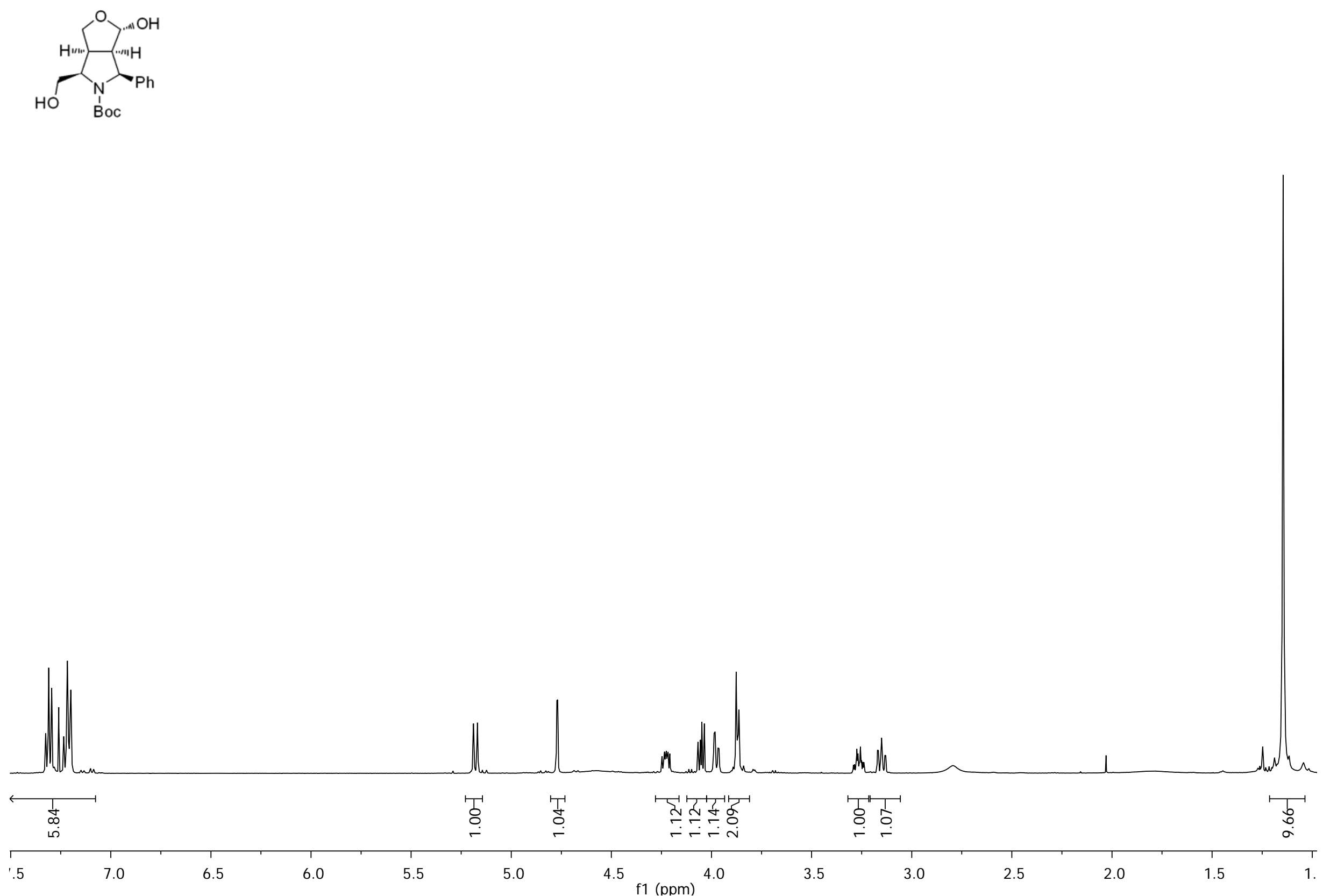
${ }^{13} \mathrm{C}-\mathrm{NMR}\left(\mathrm{CDCl}_{3}, 125.7 \mathrm{MHz}\right)$ of compound $\mathbf{1 6 b}$

$\widehat{o}$
0
$\stackrel{\circ}{0}$
1

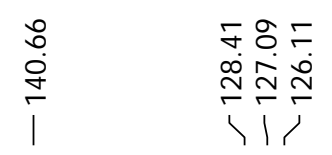

$\circ$
$\stackrel{\circ}{\circ}$
$\stackrel{+}{1}$

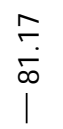

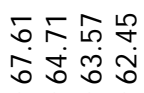

8
$\dot{0}$
$i$
1

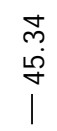

$\stackrel{\overrightarrow{0}}{\stackrel{0}{\infty}} \underset{1}{1}$

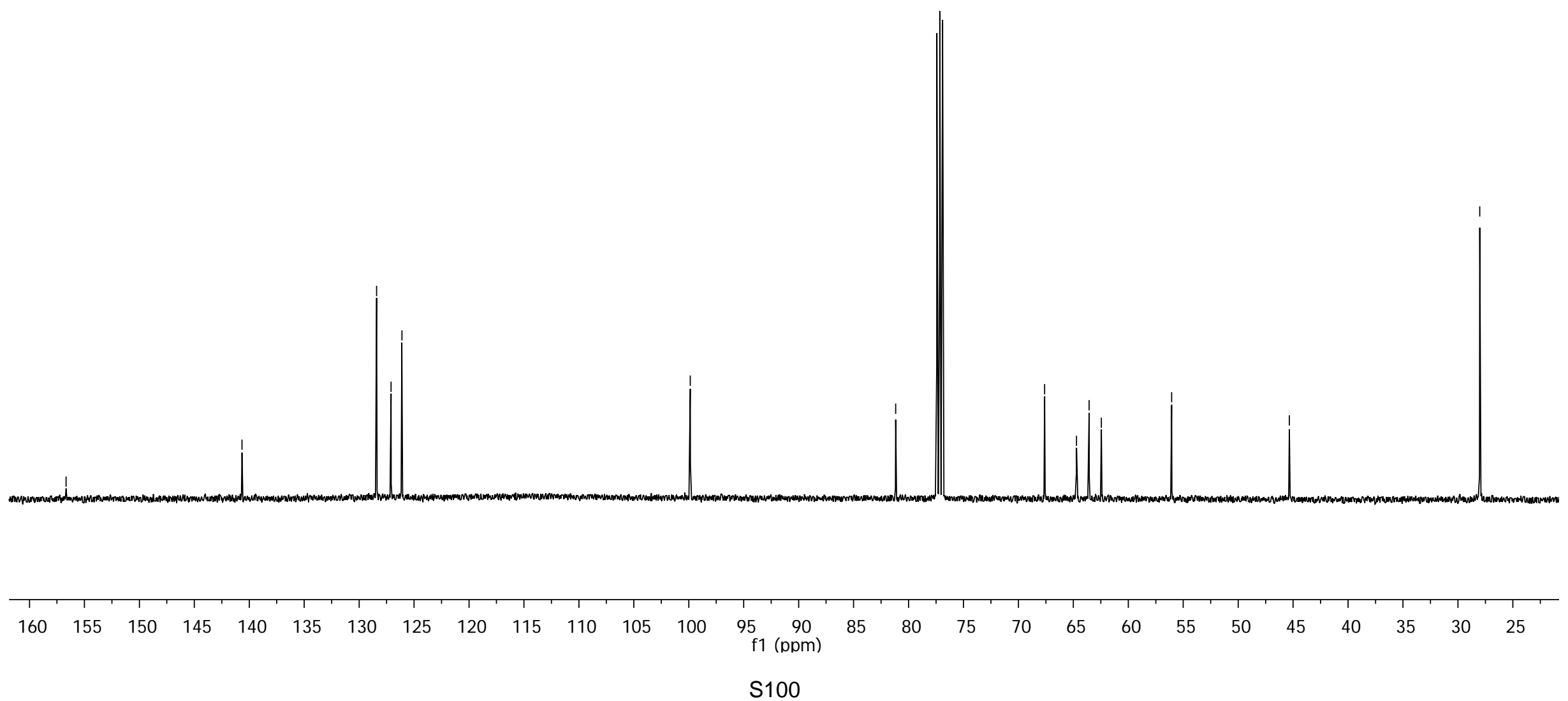


${ }^{1} \mathrm{H}-\mathrm{NMR}\left(\mathrm{CDCl}_{3}, 500 \mathrm{MHz}\right)$ of compound $\mathbf{1 7 b}$
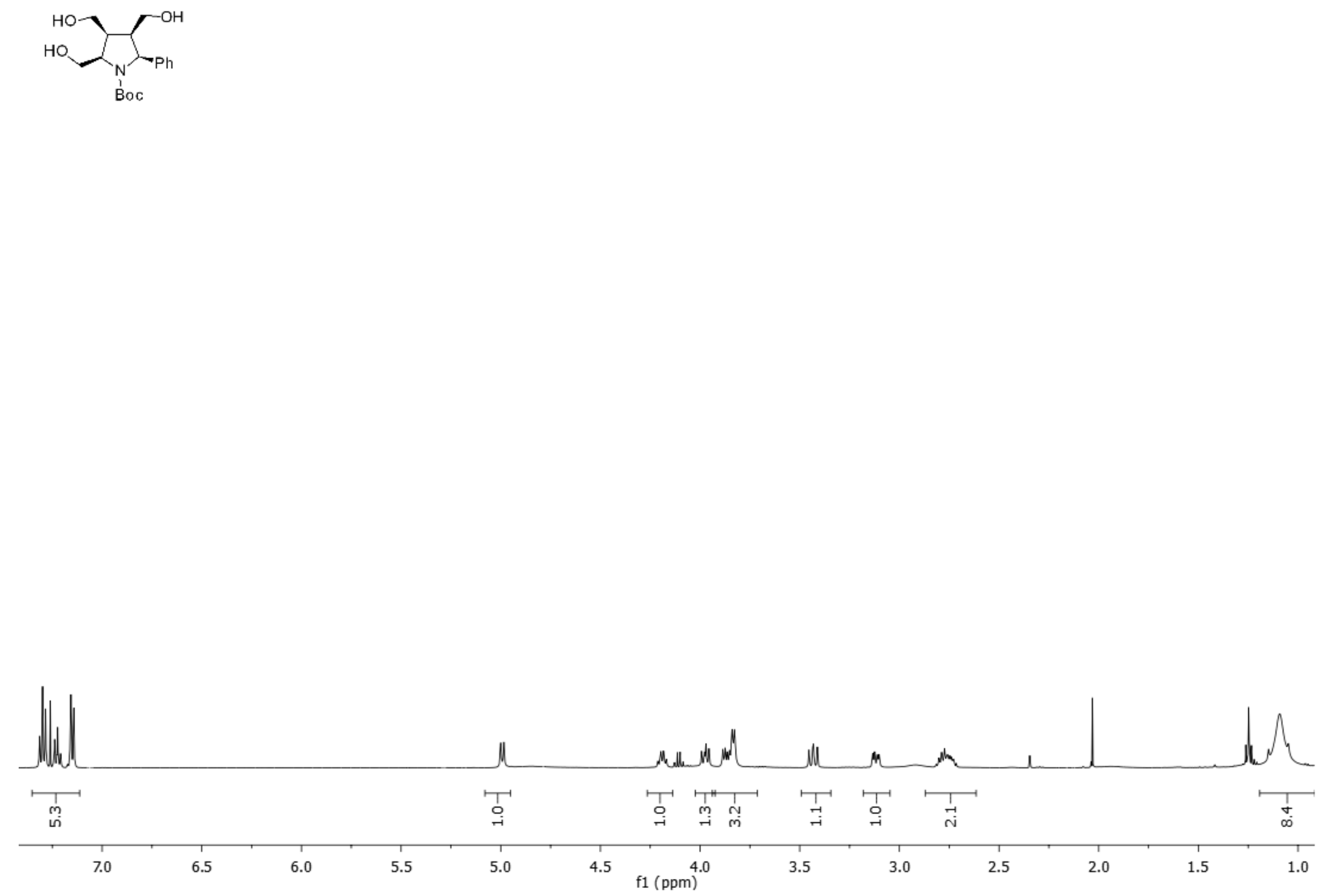
${ }^{13} \mathrm{C}-\mathrm{NMR}\left(\mathrm{CDCl}_{3}, 125.7 \mathrm{MHz}\right)$ of compound 17b

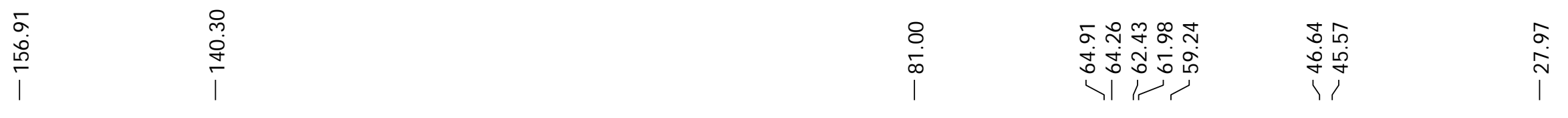

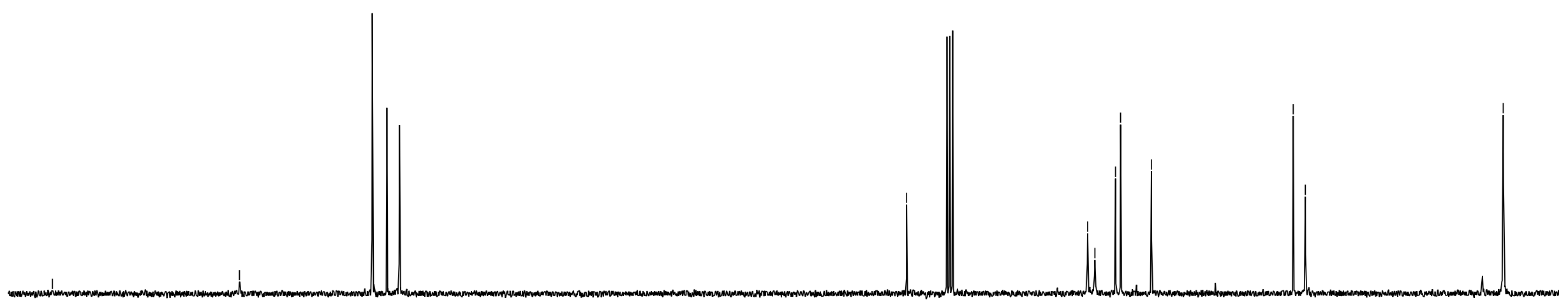

$\begin{array}{lllllllllllllllllllllllllllllllllllllllll}160 & 155 & 150 & 145 & 140 & 135 & 130 & 125 & 120 & 115 & 110 & 105 & 100 & 95 & 90 & 85 & 80 & 75 & 70 & 65 & 60 & 55 & 50 & 45 & 40 & 35 & 30 & 25\end{array}$ S102 
${ }^{1} \mathrm{H}-\mathrm{NMR}\left(\mathrm{D}_{2} \mathrm{O}, 500 \mathrm{MHz}\right)$ of compound $\mathbf{1 8 b}$

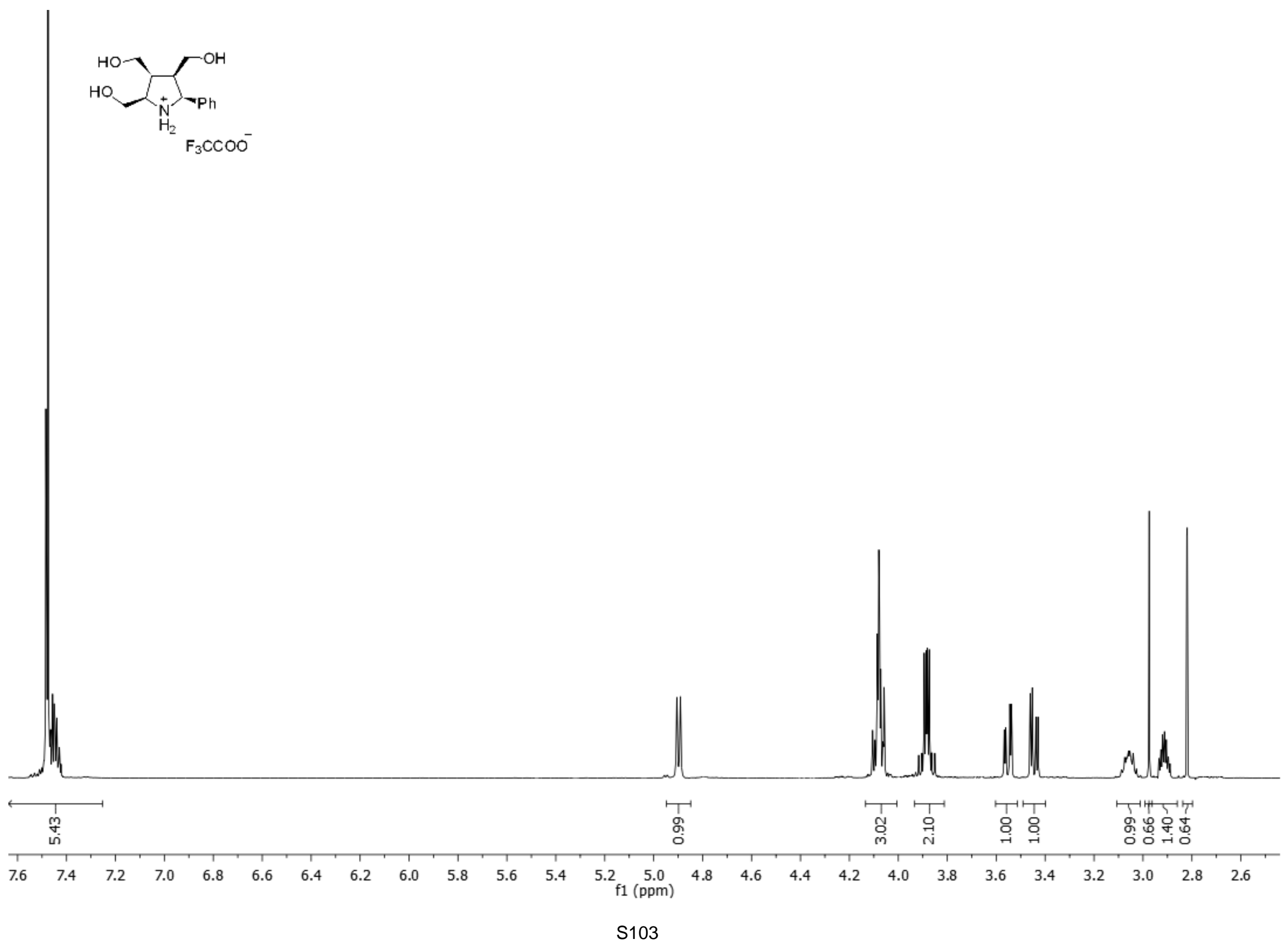


${ }^{13} \mathrm{C}-\mathrm{NMR}\left(\mathrm{D}_{2} \mathrm{O}, 125.7 \mathrm{MHz}\right)$ of compound $\mathbf{1 8 b}$

\begin{tabular}{|c|c|}
\hline 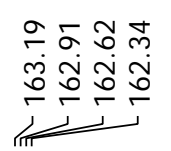 & 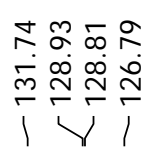 \\
\hline
\end{tabular}

$\stackrel{0}{\rightarrow}$ 노의

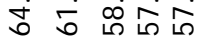

$1 \quad<1$

$\circ \stackrel{\infty}{\circ}$

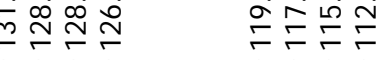

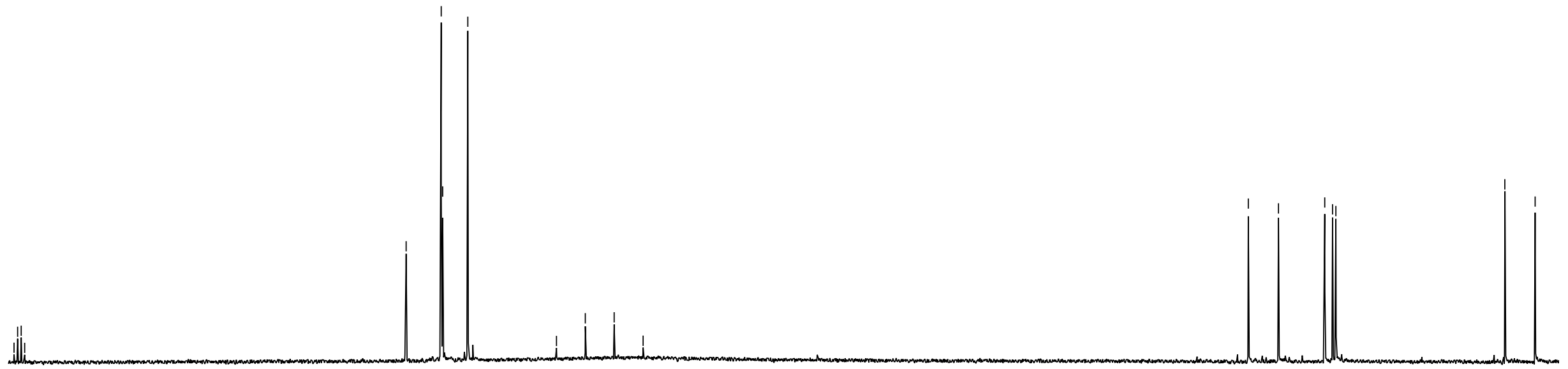

